\title{
Densidade local em grafos
}

Luis Eduardo Zambrano Fernández

Tese De Doutorado

Instituto DE MATEMÁTICA E Estatística

UNIVERSIDADE DE SÃo PAUlo

Programa: Pós-Graduação em Ciência da Computação

Orientador: Prof. Dr. Yoshiharu Kohayakawa

Durante o desenvolvimento deste trabalho o autor recebeu auxílio financeiro do $\mathrm{CNPq}$

São Paulo, dezembro de 2018. 


\section{Densidade local em grafos}

Esta versão da tese contém as correções e alterações sugeridas pela Comissão Julgadora durante a defesa da versão original do trabalho, realizada em 14/11/2018. Uma cópia da versão original está disponível no Instituto de Matemática e Estatística da Universidade de São Paulo.

Comissão Julgadora:

- Prof. Dr. Carlos Hoppen UFRGS

- Prof. Dr. Daniel Morgato Martin UFABC

- Prof. Dr. Fabricio Siqueira Benevides UFC

- Prof. Dr. Guilherme Oliveira Mota UFABC

- Prof. Dr. Yoshiharu Kohayakawa (presidente) IME-USP 


\section{Agradecimentos}

O autor agradece ao Prof. Dr. Guilherme Oliveira Mota pela sugestão de considerar o problema da densidade local em grafos com cintura ímpar grande. 


\section{Resumo}

Fernández, L. E. Z. Densidade local em grafos 2018. 45 f. Tese de doutorado - Instituto de Matemática e Estatística, Universidade de São Paulo, São Paulo, 2018.

Nós consideramos o seguinte problema. Fixado um grafo $\mathrm{H}$ e um número real $\alpha \in(0,1]$, determine o menor $\beta=\beta(\alpha, \mathrm{H})$ que satisfaz a seguinte propriedade: se $\mathrm{G}$ é um grafo de ordem $n$ no qual cada subconjunto de $\lfloor\alpha n\rfloor$ vértices induz mais que $\beta n^{2}$ arestas então $\mathrm{G}$ contém $\mathrm{H}$ como subgrafo. Este problema foi iniciado e motivado por Erdős ao conjecturar que todo grafo livre de triângulo de ordem $n$ contém um subconjunto de $\lfloor n / 2\rfloor$ vértices que induz no máximo $n^{2} / 50$ arestas.

Nosso resultado principal mostra que $i$ ) todo grafo de ordem $n$ livre de triângulos e pentágonos contém um subconjunto de $\lfloor n / 2\rfloor$ vértices que induz no máximo $n^{2} / 64$ arestas, e ii) se G é um grafo regular de ordem $n$ livre de triângulo, com grau excedendo $n / 3$, então $\mathrm{G}$ contém um subconjunto de $\lfloor n / 2\rfloor$ vértices que induz no máximo $n^{2} / 50$ arestas. Se além disso G não é 3-cromático então G contém um subconjunto de $\lfloor n / 2\rfloor$ vértices que induz menos de $n^{2} / 54$ arestas. Como subproduto e confirmando uma conjectura de Erdős assintoticamente, temos que todo grafo regular de ordem $n$ livre de triângulo com grau excedendo $n / 3$ pode ser tornado bipartido pela omissão de no máximo $(1 / 25+o(1)) n^{2}$ arestas. Nós também fornecemos um contraexemplo a uma conjectura de Erdős, Faudree, Rousseau e Schelp.

Palavras-chave: Densidade local, teorema de Turán, metades esparsas, bisecções de grafos. 


\section{Abstract}

Fernández, L. E. Z. Local density in graphs 2018. 45 f. Tese de doutorado. Instituto de Matemática e Estatística, Universidade de São Paulo, São Paulo, 2018.

We consider the following problem. Fixed a graph $\mathrm{H}$ and a real number $\alpha \in(0,1]$, determine the smallest $\beta=\beta(\alpha, \mathrm{H})$ satisfying the following property: if $\mathrm{G}$ is a graph of order $n$ such that every subset of $\lfloor\alpha n\rfloor$ vertices spans more that $\beta n^{2}$ edges then $\mathrm{G}$ contains $\mathrm{H}$ as a subgraph. This problem was initiated and motivated by Erdős who conjectured that every triangle-free graph of order $n$ contains a subset of $\lfloor n / 2\rfloor$ vertices that spans at most $n^{2} / 50$ edges.

Our main result shows that $i$ ) every triangle- and pentagon-free graph of order $n$ contains a subset of $\lfloor n / 2\rfloor$ vertices inducing at most $n^{2} / 64$ edges and, ii) if $\mathrm{G}$ is a triangle-free regular graph of order $n$ with degree exceeding $n / 3$ then $\mathrm{G}$ contains a subset of $\lfloor n / 2\rfloor$ vertices inducing at most $n^{2} / 50$ edges. Furthermore, if $\mathrm{G}$ is not 3 -chromatic then $\mathrm{G}$ contains a subset of $\lfloor n / 2\rfloor$ vertices inducing less than $n^{2} / 54$ edges. As a by-product and confirming a conjecture of Erdös asymptotically, we obtain that every $n$-vertex triangle-free regular graph with degree exceeding $n / 3$ can be made bipartite by removing at most $(1 / 25+o(1)) n^{2}$ edges. We also provide a counterexample to a conjecture of Erdős, Faudree, Rousseau and Schelp.

Keywords: Local density, Turán's theorem, sparse-halves, bisections of graphs. 


\section{Sumário}

$\begin{array}{ll}\text { Agradecimientos } & \text { i }\end{array}$

$\begin{array}{lll}\text { Resumo } & \text { iii }\end{array}$

$\begin{array}{lll}\text { Abstract } & \text { v }\end{array}$

Sumário $\quad$ viii

$\begin{array}{ll}\text { Lista de Símbolos } & \text { ix }\end{array}$

Lista de Figuras $\quad$ xiii

1 Introdução 1

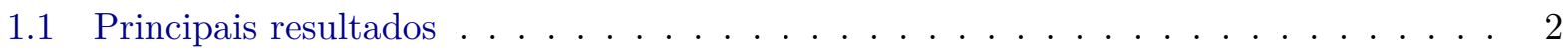

1.2 Notação . . . . . . . . . . . . . . . . . . . . . . . . . . . . . . . 6

1.3 Extensões e problemas relacionados . . . . . . . . . . . . . . . 7

1.3.1 Problemas tipo Turán-Ramsey . . . . . . . . . . . . . . . . . . 7

1.3.2 O Teorema de Turán . . . . . . . . . . . . . . . . . . . . . . 8

1.3.3 Número de pentágonos e diferença com o subgrafo bipartido máximo . . . . . 9

1.3.4 Max-Cut e partições judiciosas . . . . . . . . . . . . . . . . . . . . . 11

2 Grafos livres de triângulos e pentágonos $\quad 13$

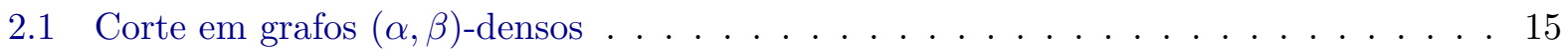

2.2 Prova do Teorema $1.3 \ldots \ldots \ldots \ldots \ldots \ldots \ldots$

2.3 Grafos livres de triângulo com grau médio acotado . . . . . . . . . . . . . 17

3 Grafos densos livres de triângulo 
viii SUMÁRIO

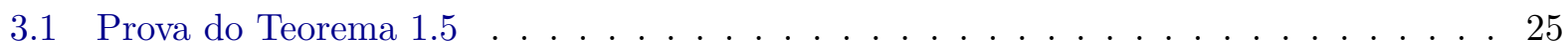

4 Uma conjectura de Erdős, Faudree, Rousseau e Schelp 29

4.1 Grafos de Ramsey . . . . . . . . . . . . . . . . . . . . . . . 29

4.2 Um contraexemplo para a Conjectura $1.7 \ldots \ldots$. . . . . . . . . . . 30

5 Conclusões e considerações finais $\quad 33$

$\begin{array}{ll}\text { A Prova computacional do Teorema } 1.8 & 35\end{array}$

$\begin{array}{ll}\text { Referências Bibliográficas } & 39\end{array}$ 


\section{Lista de Símbolos}

\section{Parâmetros de um grafo}

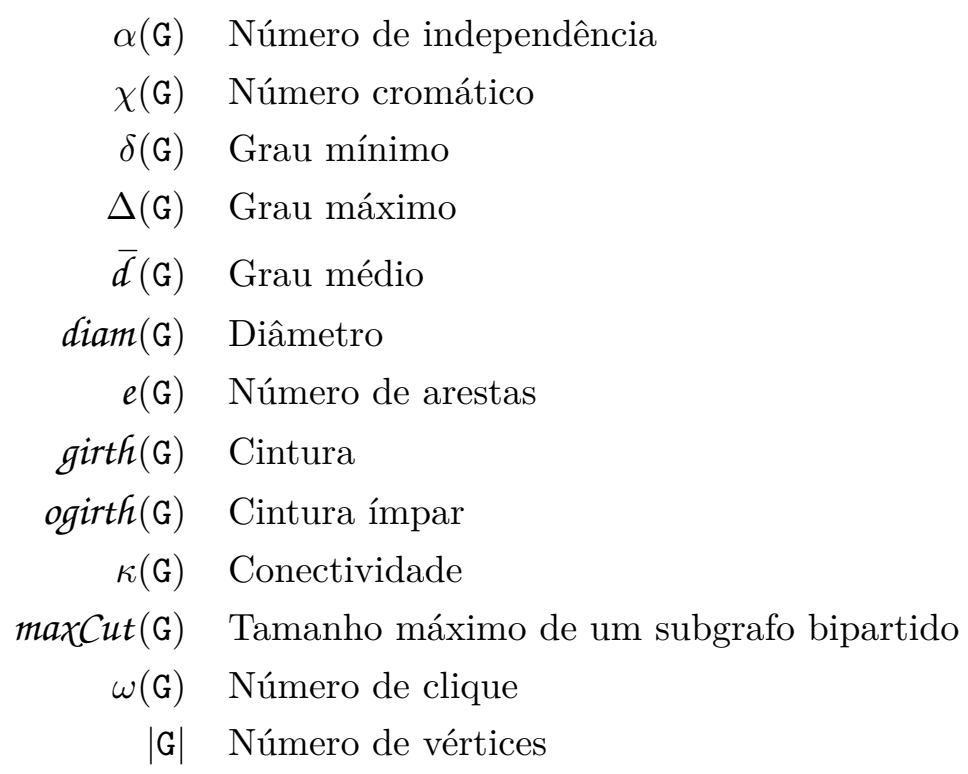




\title{
Família de Grafos
}

\author{
$\mathrm{C}_{n} \quad$ Ciclo de $n$ vértices \\ $\mathrm{G}^{\mathrm{C}}$ Grafo complemento de G \\ GCle Grafo de Clebsch \\ GGew Grafo de Gewirtz \\ GGrö Grafo de Grötzsch \\ aHiSi Grafo de Higman-Sims \\ aHoSi Grafo de Hoffman-Singleton \\ GKne $_{n, k} \quad$ Grafo de Kneser de $\left(\begin{array}{l}n \\ k\end{array}\right)$ vértices \\ GMöb $_{n}$ Grafo escada de Möbius de ordem $n$ \\ GWag Grafo de Wagner \\ $\Gamma_{i} \quad$ Grafo de Andrásfai $i$-regular \\ $\Gamma_{i, k} \quad(i, k)$-grafo de Andrásfai generalizado \\ $\mathrm{H}^{[n]}$ Blow-up balanceado de $n$ vértices do grafo $\mathrm{H}$ \\ $\mathrm{K}_{r} \quad$ Grafo completo de $r$ vértices \\ $\mathrm{K}_{r, s} \quad$ Grafo bipartido completo com partições de tamanho $r$ e $s$ \\ GPet Grafo de Petersen \\ GPet/e Grafo de Petersen com uma aresta contraída \\ $\operatorname{srg}(n, k, \lambda, \mu) \quad$ Grafos fortemente regulares com parâmetros $(n, k, \lambda, \mu)$ \\ $\mathrm{T}_{n, r} \quad$ Grafo de Turán de $n$ vértices com $r$ partições \\ $\Upsilon_{i} \quad$ Grafo Vega de $3 i+7$ vértices
}




\section{Outros símbolos}

A(G) Matriz de adjacência do grafo G

$d(v) \quad$ Grau do vértice $v$

$e(U, W) \quad$ Número de arestas com um extremo em $U$ e outro em $W$

$E(\mathrm{G})$ Conjunto de arestas de G

$\mathbb{E}[X] \quad$ Valor esperado da variável aleatória $X$

$\left.\mathrm{G}\right|_{U} \quad$ Subgrafo de $\mathrm{G}$ induzido pelo conjunto $U$

$(\mathrm{H}, w)$ Grafo ponderado $\mathrm{H}$ com função peso $w$

$\mathrm{H} \subseteq \mathrm{G} \quad$ H é subgrafo de $\mathrm{G}$

$[k]$ Conjunto de inteiros $\{1, \ldots, k\}$

$\lambda_{i}(A) \quad i$-ésimo maior autovetor da matriz $A$

L(G) Matriz Laplaciana do grafo G

$\|\phi\| \quad$ Norma Euclidiana do vetor $\phi$

$\|M\|_{o p}$ Operador norma de uma matriz $M$

$\mathbb{P}[E] \quad$ Probabilidade do evento $E$

$\langle\phi, \psi\rangle \quad$ Produto interior de $\phi$ e $\psi$

Q(G) Matriz Laplaciana sem sinal de G

$\mathcal{S}(v) \quad$ Classe de similaridade do vértice $v$

$s(v)$ Tamanho da classe de similaridade do vértice $v$

$V(\mathrm{G})$ Conjunto de vértices de $\mathrm{G}$

$\left\{\begin{array}{l}X \\ k\end{array}\right\} \quad$ Família de subconjuntos de $X$ de tamanho $k$ 


\section{Lista de Figuras}

1 Blow-up balanceado do pentágono e do grafo de Petersen sobre $n=10 k$ vértices . . 2

2 Melhor cota inferior para $\beta\left(\alpha, \mathrm{K}_{3}\right)$ conhecida nesta data $\ldots \ldots \ldots \ldots$

3 Exemplos de grafos livres de triângulo não bipartidos . . . . . . . . . . . . . . . . . 10

$4 \quad$ Grafos de Andrásfai $\Gamma_{i},(i=1 \ldots 4) \ldots \ldots \ldots \ldots \ldots \ldots$

5 Estrutura global de um grafo Vega . . . . . . . . . . . . . . . . . . 23

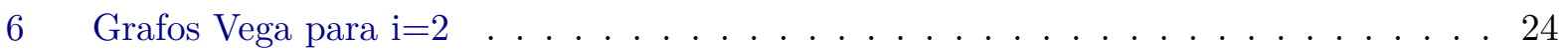

7 Blow-up do grafo de Grötzsch de 29 vértices . . . . . . . . . . . . . . . . . 25 


\section{Capítulo 1}

\section{Introdução}

Matemática Discreta é a disciplina que lida com objetos matemáticos enumeráveis (como conjuntos, vetores, matrizes, funções, grafos, etc.), suas propriedades e as relações entre essas propriedades. A teoria de grafos extremais, um recente subtópico da matemática discreta, tem experimentado um significativo crescimento nas últimas décadas devido principalmente a sua rica família de conjecturas, problemas, técnicas e aplicações em ciência da computação, otimização combinatória, física, química, entre outras. ${ }^{1}$ Enquanto isso, o estudo de grandes grafos, espaços vetoriais e análise espectral estão consideradas como teorias fundamentais no suporte para o futuro da ciência da computação (cf. Hopcroft, Soundarajan e Wang [HSW11]).

No presente trabalho, estudamos o problema da densidade local em grafos, um problema da teoria de grafos extremais que, fixado um real $0<\alpha \leq 1$ e um grafo $\mathrm{H}$, pergunta pelo menor real $\beta=\beta(\alpha, \mathrm{H})$ que satisfaz a seguinte propriedade: se $\mathbf{G}$ é um grafo de $n$ vértices no qual cada subconjunto de $\lfloor\alpha n\rfloor$ vértices induz mais que $\beta n^{2}$ arestas então $\mathrm{G}$ contém $\mathrm{H}$ como subgrafo. Por exemplo, o teorema de Mantel [Man07] garante que $\beta\left(1, \mathrm{~K}_{3}\right)=1 / 4$, onde $\mathrm{K}_{3}$ é o triângulo (o grafo completo de três vértices). Este problema foi iniciado e motivado em 1976 pelo célebre matemático húngaro Paul Erdős [Erd76], que para o caso particular $\alpha=1 / 2, \mathrm{H}=\mathrm{K}_{3}$, perguntou se vale a igualdade $\beta=1 / 50$.

Problema 1.1. (Erdős $[\operatorname{Erd} 76])$.

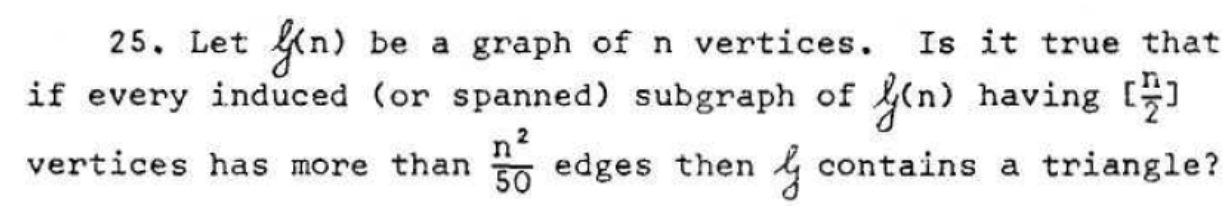

A cota inferior $\beta\left(1 / 2, \mathrm{~K}_{3}\right) \geq 1 / 50$ é fornecida, por exemplo, pelo grafo de Petersen aPet, um grafo livre de triângulo com dez vértices no qual o mínimo sobre o número de arestas de subgrafos induzidos sobre cinco vértices é igual a dois. De fato, blow-ups balanceados de aPet e do pentágono $\mathrm{C}_{5}$ sobre $n=10 k$ vértices, com $k$ um inteiro positivo, são famílias infinitas de grafos que fornecem $\beta\left(1 / 2, \mathrm{~K}_{3}\right) \geq 1 / 50$, (veja Figura 1 ). Um blow-up sobre $n$ vértices de um grafo $\mathrm{H}$ é o grafo que resulta de substituir cada vértice $v$ em $\mathrm{H}$ por um conjunto independente $I(v)$ satisfazendo $n=\sum_{v \in V(\mathrm{H})}|I(v)|$, e cada aresta $u v$ de $\mathrm{H}$ por um grafo bipartido completo com partições $I(u)$ e $I(v)$. O blow-up é balanceado se as partições tem tamanhos tão iguais como seja possível. Neste caso escrevemos $\mathrm{H}^{[n]}$.

\footnotetext{
${ }^{1} \mathrm{~A}$ teoria de grafos foi outrora considerada como "tipos de problemas combinatórios de natureza estrutural" e portanto, parte da combinatória (tópico da matemática discreta que surgiu no século XVII com os trabalhos de Blaise Pascal, Pierre de Fermat, Jakob Bernoulli e Gottfried Leibniz). No entanto, os primórdios da teoria de grafos podem ser situados no trabalho de Leonard Euler "Solutio problematis ad geometriam situs pertinentis" apresentado em 1735 na Academia de Ciências de São Petersburgo. Este é um dos artigos fundacionais da matemática moderna; contém, por exemplo, a primeira prova de que a soma dos graus dos vértices de um grafo é igual ao duplo do número de arestas e registra o descobrimento da primeira propriedade topológica: a caraterística de Euler. Para uma breve revisão histórica veja Baranov e Stechkin [BS95, p. 1] e Shields [Shi12]).
} 

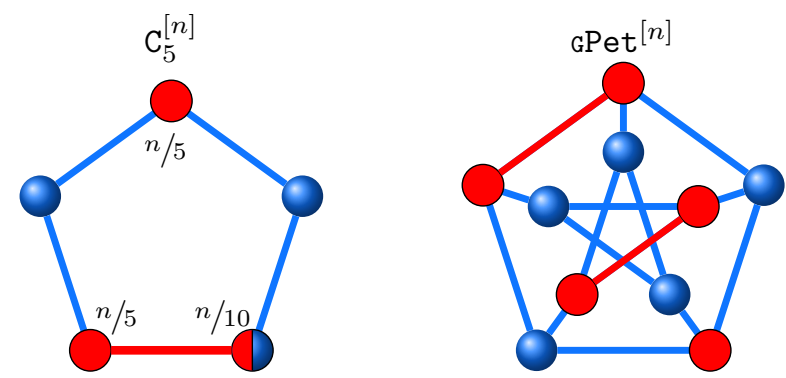

Figura 1: Blow-up balanceado do pentágono e do grafo de Petersen sobre $n=10 k$ vértices

Para um número real $\alpha \in(0,1]$ e um grafo G com $n$ vértices, o inteiro $e_{\alpha}(\mathrm{G})$ é o mínimo sobre o número de arestas induzidas por subconjuntos de $\lfloor\alpha n\rfloor$ vértices de G, i.e.

$$
e_{\alpha}(\mathrm{G}) \stackrel{\text { def }}{=} \min \{e(U): U \subseteq V(\mathrm{G}),|U|=\lfloor\alpha n\rfloor\} .
$$

O quociente $e_{\alpha}(\mathrm{G}) / n^{2}$ é a $\alpha$-densidade local de G. Assim, a questão no Problema 1.1 é, se para $\alpha=1 / 2$, existem grafos livres de triângulo com $\alpha$-densidade local estritamente maior aos blow-ups balanceados (sobre $10 k$ vértices) de $C_{5}$ e GPet? Erdős conjecturou que a resposta a esta pergunta é negativa, i.e. que, de fato, vale a igualdade $\beta\left(1 / 2, \mathrm{~K}_{3}\right)=1 / 50$.

Conjectura 1.2 (Erdős, 1976 [Erd76, Problem 25]). Todo grafo G de ordem $n$ livre de triângulo contém um subconjunto de $\lfloor n / 2\rfloor$ vértices que induz no máximo $n^{2} / 50$ arestas.

Nesta data, a Conjectura 1.2 permanece aberta. ${ }^{2}$

\subsection{Principais resultados}

Neste documento estamos especialmente interessados em determinar condições para as quais um grafo G livre de triângulo de ordem $n$ contém um subgrafo induzido de ordem $\lfloor n / 2\rfloor$ com no máximo $n^{2} / 50$ arestas. Tipicamente, considera-se uma propriedade adicional no grafo G de importância combinatória (enfraquecendo assim a Conjectura 1.2), e provamos que, nestas condições, G satisfaz a relação

$$
e_{1 / 2}(G) \leq n^{2} / 50 \text {. }
$$

Primeiro, nós consideramos grafos cujas propriedades estruturais "se afastam" das propriedades

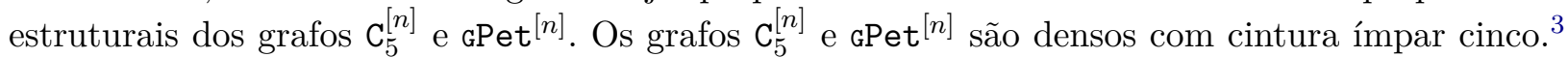
Para tanto, considera-se grafos com cintura ímpar estritamente maior que cinco, como também grafos livres de triângulo com grau médio acotado. Prova-se que, em ambos os casos, a relação (1.1) vale.

\section{Grafos livres de triângulos e pentágonos}

Claramente, um grafo G é livre de triângulo se e só se ogirth $(G) \geq 5$. Nós mostramos que se a desigualdade é estrita então $e_{1 / 2}(\mathrm{G}) \leq n^{2} / 64$.

Teorema 1.3. Se G é um grafo de $n$ vértices e cintura ímpar ogirth(G) > 5. Então, G contém $\lfloor n / 2\rfloor$ vértices que induzem no máximo $n^{2} / 64$ arestas.

\footnotetext{
${ }^{2}$ Num artigo de publicação póstuma, Erdős [Erd97, Problem 1] encorajou a comunidade acadêmica a não esquecer várias de suas conjecturas e, como costumava, ofereceu uma simbólica recompensa monetária de US $\$ 250$ pela prova ou refutação da Conjectura 1.2. Para alguns autores, isto é uma indicação sólida que esta conjectura é altamente não trivial (cf. e.g. [Sud11, §5.1]).

${ }^{3} \mathrm{O}$ tamanho do menor ciclo ímpar num grafo G é a sua cintura ímpar ogirth(G), definida $\infty$ se G não contém ciclos ímpares. Uma sequência de grafos $\left(\mathrm{G}_{n}\right)$ é densa se $\left|\mathrm{G}_{n}\right| \rightarrow \infty$ e $e\left(\mathrm{G}_{n}\right)=\Omega\left(\left|\mathrm{G}_{n}\right|^{2}\right)$.
} 
O Teorema 1.3 complementa um recente resultado de Bedenknecht, Mota, Reiher e Schacht [BMRS17] que provam que se G é homomorfo a algum grafo de Andrásfai generalizado $\Gamma_{i, k}$ então $\boldsymbol{e}_{1 / 2}(\mathrm{G}) \leq n^{2} /\left(2(2 k+1)^{2}\right)$. O grafo de Andrásfai generalizado $\Gamma_{i, k}$, com $i, k \geq 2$ inteiros, é um grafo $i$-regular livre de triângulo de cintura ímpar $2 k+1$ (cf. Definição 2.6).

A constante 1/64 no Teorema 1.3 poderia não ser justa. Em particular, é mais fraca que 1/98, o valor que segue do resultado em [BMRS17] antes mencionado, para $k=3$. Contudo, e em contraste com o resultado em [BMRS17], a cota $e_{1 / 2}(\mathrm{G}) \leq n^{2} / 64$ que garante o Teorema 1.3 independe do homomorfismo de G.

\section{Grafos com grau médio acotado}

Para grafos livres de triângulo de ordem $n$ com grau médio acotado, prova-se em particular que a condição $\bar{d}(\mathrm{G}) \leq \frac{7}{40} n$ implica (1.1). Por causa da relação $\bar{d}(\mathrm{G})=2 e(\mathrm{G}) / n$, resultados sobre o número de arestas $e(G)$ de $\mathrm{G}$ tem seus equivalentes enunciados para o grau médio $\bar{d}(\mathrm{G})$ de $\mathrm{G}$.

Proposição 1.4. Seja G um grafo livre de triângulos de ordem $n$ com no máximo $\frac{7}{80} n^{2}$ arestas. Então G contém um subgrafo induzido de ordem $\lfloor n / 2\rfloor$ com no máximo $n^{2} / 50$ arestas.

A Proposição 1.4 melhora um resultado em Keevash e Sudakov [KS06] onde mostra-se que a relação (1.1) vale para grafos livres de triângulo com $e(G) \leq \frac{1}{12} n^{2}$ ou $e(G) \geq \frac{1}{5} n$.

\section{Grafos densos livres de triângulos}

Em segundo lugar, considera-se grafos livres de triângulo com grau mínimo $\delta(\mathrm{G})>n / 3$. Como usual, $n=|\mathrm{G}|$ é o número de vértices de G. Devido a um resultado de Brandt e Thomassé [BT10] sabe-se que grafos com $\delta(G)>n / 3$ admitem uma partição de seus vértices em no máximo quatro conjuntos independentes, i.e. $\chi(\mathrm{G}) \leq 4$. Este importante resultado resolve um problema de Erdős e Simonovits [ES73].

Como todo grafo bipartido de ordem $n$ contém um conjunto independente de cardinalidade pelo menos $n / 2$, o caso $\chi(\mathrm{G}) \leq 2$ satisfaz (1.1) trivialmente. $\mathrm{O}$ caso $\chi(\mathrm{G})=3$ segue dos resultados de Jin [Jin93], Chen, Jin e Koh [CJK97] e do resultado principal em Bedenknecht, Mota, Reiher e Schacht [BMRS17]. Isto é, se G é livre de triângulo com grau mínimo $\delta(G)>n / 3$ e número cromático $\chi(\mathrm{G})=3$ então $e_{1 / 2}(\mathrm{G}) \leq n^{2} / 50$.

Jin [Jin93] mostrou que, se G é um grafo de ordem $n$ livre de triângulo com $\delta(G)>\frac{i+1}{3 i+2} n$, para algum $1 \leq i \leq 9$, então $\mathrm{G} \rightarrow \Gamma_{i}$, i.e. G é homomorfo ao grafo de Andrásfai $\Gamma_{i}$ (cf. Definição 2.2). Chen, Jin e Koh [CJK97] provaram que um grafo G de ordem $n$ livre de triângulo com $\delta(G)>n / 3$ é homomorfo a algum grafo de Andrásfai $\Gamma_{i}$, e portanto $\chi(\mathrm{G}) \leq 3$, ou contém o grafo de Grötzsch GGrö como subgrafo, e portanto $\chi(G) \geq 4$ (veja Brandt [Bra99] para uma prova mais simples deste resultado). O grafo de Grötzsch GGrö (v. Figura 3) é o único grafo livre de triângulo com número cromático quatro de menor ordem possível (cf. Chvátal [Chv74]). Assim, destes resultados temos que, se G é livre de triângulo com $\delta(\mathrm{G})>n / 3$ e $\chi(\mathrm{G})=3$ então $\mathrm{G} \rightarrow \Gamma_{i}$. Por outro lado, o resultado em [BMRS17] garante que, se G $\rightarrow \Gamma_{i}$ então G satisfaz (1.1).

Para grafos livres de triângulo com $\delta(\mathrm{G})>n / 3$ e $\chi(\mathrm{G})=4$ nós mostramos que a relação $e_{1 / 2}(G)<n^{2} / 54$ é satisfeita sempre que G é regular. Isso permite enunciar o seguinte resultado.

Teorema 1.5. Seja G um grafo regular de ordem $n$ livre de triângulo. Se o grau de G excede $n / 3$ então $e_{1 / 2}(\mathrm{G}) \leq n^{2} / 50$. Se além disso, G não é 3 -cromático, então $e_{1 / 2}(\mathrm{G})<n^{2} / 54$.

Observe que no Teorema 1.5 a desigualdade é estrita se $\chi(G) \neq 3$. Nós conjecturamos que o enunciado do Teorema 1.5 continua valendo quando a condição de regularidade de G é suprimida 
(cf. Conjectura 5.1). Além disso, conjecturamos que a constante 1/54 neste teorema é o melhor possível (cf. Conjectura 5.2).

Em 1971, Erdős [Erd71] conjecturou que todo grafo livre de triângulo com $n$ vértices pode ser tornado bipartido ao remover no máximo $n^{2} / 25$ arestas. Nesta data, esta conjectura permanece aberta. Como no grafo $C_{5}^{[5 k]}$ precisamos remover $k^{2}$ arestas para virar-lhe bipartido, a constante $1 / 25$ nesta conjectura não pode ser decrementada. Nós verificamos esta conjectura assintoticamente para grafos que satisfazem as condições do Teorema 1.5.

Corolário 1.6. Seja G um grafo regular de ordem $n$ livre de triângulo. Se o grau de G excede $n / 3$ então G pode ser tornado bipartido pela remoção de no máximo $\left(\frac{1}{25}+o(1)\right) n^{2}$ arestas.

\section{Uma conjectura de Erdős, Faudree, Rousseau e Schelp}

Em 1994, Erdős, Faudree, Rousseau e Schelp [EFRS94] estudaram o Problema 1.1 ao substituir $\lfloor n / 2\rfloor$ por $\lfloor\alpha n\rfloor$, com $\alpha \in(0,1]$, e $n^{2} / 50$ por $\beta n^{2}$, com $\beta=\beta\left(\alpha, \mathrm{K}_{3}\right)$ um valor ótimo conjecturado. Para tanto, foram considerados os blow-ups balanceados dos grafos $\mathrm{K}_{2}$, o pentágono $\mathrm{C}_{5}$ e o grafo de Wagner GWag (o grafo de Wagner é o grafo GWag que resulta de adicionar as quatro arestas entre vértices opostos no octógono $\left.\mathrm{C}_{8}\right)$. Estes grafos fornecem as seguintes cotas inferiores para $\beta\left(\alpha, \mathrm{K}_{3}\right)$ :

(i) No grafo $\mathrm{K}_{2}^{[n]}$, se $1 / 2<\alpha \leq 1$ então

$$
\beta\left(\alpha, \mathrm{K}_{3}\right) \geq \frac{1}{2}\left(\alpha-\frac{1}{2}\right)=\frac{2 \alpha-1}{4} .
$$

(ii) No grafo $\mathrm{C}_{5}^{[n]}$, se $2 / 5<\alpha \leq 3 / 5$ então

$$
\beta\left(\alpha, \mathrm{K}_{3}\right) \geq \frac{1}{5}\left(\alpha-\frac{2}{5}\right)=\frac{5 \alpha-2}{25} .
$$

(iii) No grafo GWag ${ }^{[n]}$, se $3 / 8<\alpha \leq 1 / 2$ então

$$
\beta\left(\alpha, \mathrm{K}_{3}\right) \geq \frac{1}{8}\left(\alpha-\frac{3}{8}\right)=\frac{8 \alpha-3}{64} .
$$

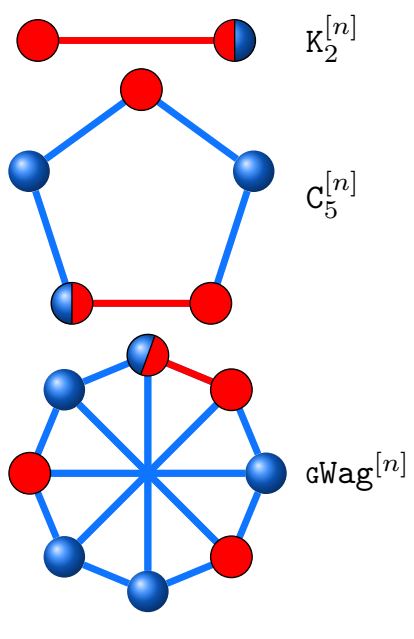

Claramente, $\frac{2 \alpha-1}{4} \geq \frac{5 \alpha-2}{25}$ se $\frac{17}{30} \leq \alpha \leq 1$, e $\frac{5 \alpha-2}{25} \geq \frac{8 \alpha-3}{64}$ se $\frac{53}{120} \leq \alpha \leq \frac{17}{30}$. Os autores em [EFRS94] conjecturaram que o grafo $\mathrm{K}_{2}^{[n]}$ fornece o valor ótimo para $\beta\left(\alpha, \mathrm{K}_{3}\right)$ se $\alpha \in\left[\frac{17}{30}, 1\right]$ e o grafo $\mathrm{C}_{5}^{[n]}$ fornece o valor ótimo para $\beta\left(\alpha, \mathrm{K}_{3}\right)$ se $\alpha \in\left[\frac{53}{120}, \frac{17}{30}\right){ }^{4}$

Conjectura 1.7 (Erdős, Faudree, Rousseau e Schelp, 1994 [EFRS94]).

$$
\beta\left(\alpha, \mathrm{K}_{3}\right)= \begin{cases}\frac{2 \alpha-1}{4} & \text { se } 17 / 30 \leq \alpha \leq 1 \\ \frac{5 \alpha-2}{25} & \text { se } 53 / 120 \leq \alpha<17 / 30 .\end{cases}
$$

A Conjectura 1.2 é uma instância da Conjectura 1.7 para $\alpha=1 / 2$. Esta é, de fato, a instância mais motivada por Erdős (cf. Erdős [Erd97, Problem 1], Brandt [Bra98, p. 18] ou Sudakov [Sud11, p. 2595]).

Devido ao Teorema de Mantel [Man07], que estabelece que todo grafo livre de triângulo com $n$ vértices contém no máximo $\left\lfloor n^{2} / 4\right\rfloor$ arestas, sendo o grafo bipartido $\mathrm{K}_{2}^{[n]}$ o único grafo extremal, temos que $\beta\left(1, \mathrm{~K}_{3}\right)=1 / 4$, verificando assim a Conjectura 1.7 para $\alpha=1$.

${ }^{4} 53 / 120 \approx 0.4417$ e $17 / 30 \approx 0.5667$. 
Erdős, Faudree, Rousseau e Schelp [EFRS94] mostraram que a Conjectura 1.7 verifica-se para todo $0.648 \leq \alpha \leq 1$. Além disso, que $\beta\left(1 / 2, \mathrm{~K}_{3}\right)<\frac{\sqrt{65}-7}{32}(<1 / 30)$. Estes resultados foram melhorados por Krivelevich [Kri95], que provou que a Conjectura 1.7 vale para todo $0.6 \leq \alpha \leq 1$ como também que $\beta\left(1 / 2, \mathrm{~K}_{3}\right)<1 / 36$. Krivelevich mostra além disso que, se $\mathrm{G}$ é um grafo $d$-regular de ordem $n$ livre de triângulo, com grau $d \geq 2 n / 5$, então $e_{1 / 2}(\mathrm{G}) \leq n^{2} / 50$ (cf. [Kri95, Theorem 3 ]). Observe que o Teorema 1.5 melhora este resultado ao substituir a condição $d \geq 2 n / 5$ por $d>n / 3$.

Em [Bra98], Brandt provou que para todo $\alpha \in(0,1]$, temos

$$
\beta\left(\alpha, \mathrm{K}_{3}\right) \geq \frac{1000 \alpha-326}{10000} .
$$

Como $\frac{1000 \alpha-326}{10000}>\frac{5 \alpha-2}{25}$ se $0<\alpha<474 / 1000$, este resultado é um contraexemplo para a Conjectura 1.7 no intervalo 53/120 $\leq \alpha<474 / 1000$. A cota (1.6) em [Bra98] é fornecida pelo grafo de Higman-Sims GHiSi, o único grafo fortemente regular com parâmetros $(100,22,0,6) .{ }^{5} \mathrm{Um}$ grafo fortemente regular com parâmetros $(n, d, \lambda, \mu)$ é um grafo $d$-regular de ordem $n$ no qual pares de vértices adjacentes tem $\lambda$ vizinhos comuns e pares de vértices não adjacentes tem $\mu$ vizinhos comuns. Assim, $\lambda=0$ em grafos livres de triângulo fortemente regulares.

Numa prova computacional, Brandt, Brinkmann e Harmuth [BBH00] mostraram que todo grafo $G$ aresta maximal livre de triângulo de ordem $|G| \leq 24$ satisfaz $e_{1 / 2}(G) \leq|G|^{2} / 50 .{ }^{6}$ Um grafo livre de triângulo aresta maximal é um grafo livre de triângulo para o qual a adição de uma aresta entre qualquer par de vértices não adjacentes cria um triângulo. Estes são precisamente grafos $\mathrm{K}_{3}$-livres com diâmetro dois. Todo grafo $\mathrm{K}_{3}$-livre $\mathrm{H}$ com $n$ vértices é subgrafo de um grafo $\mathrm{K}_{3}$-livre $\mathrm{G}$, com $\operatorname{diam}(\mathrm{G})=2$, da mesma ordem (e.g. adicionando arestas em $\mathrm{H}$ consecutivamente entre pares de vértices a distância pelo menos três. Observe que o supergrafo G não é único em geral). Além disso, se $e_{\alpha}(\mathrm{G}) \leq \beta n^{2}$ então $e_{\alpha}(\mathrm{H}) \leq \beta n^{2}$ para $\alpha \in(0,1]$. Portanto, o resultado em [BBH00] implica que a Conjectura 1.7 verifica-se para a instância $\alpha=1 / 2$ em grafos livres de triângulo (não necessariamente aresta maximal) de ordem $n \leq 24$. Porém, nós observamos que blow-ups balanceados do grafo de Clebsch $\mathrm{GCle}$, o único grafo fortemente regular com parâmetros $(16,5,0,2)$, fornecem um contraexemplo à Conjectura 1.7 para determinados valores de $\alpha<1 / 2$. Nossa prova é assistida por um programa de computador.

Em contraste com a relação (1.6) (provada em Brandt [Bra98]), que fornece um contraexemplo para a Conjectura 1.7, nós enfatizamos o seguinte fato: existe $\varepsilon>0$ tal que para valores de $\alpha \leq \frac{53}{120}+\varepsilon$ temos

$$
\beta\left(\alpha, \mathrm{K}_{3}\right) \geq \frac{4 \alpha-1}{64}>\frac{1000 \alpha-326}{10000} .
$$

Por outro lado, e, em contraste com a Conjectura 1.7, existe um $\varepsilon^{\prime}>\varepsilon$ tal que para valores $\alpha \leq \frac{53}{120}+\varepsilon^{\prime}$, temos que $\frac{4 \alpha-1}{64}>\frac{5 \alpha-2}{25}$. Este resultado fornece a melhor cota inferior para $\beta\left(\alpha, \mathrm{K}_{3}\right)$, conhecida nesta data, para pequenos valores de $\alpha$ (veja Figura 2).

\section{Teorema 1.8 .}

$$
\beta\left(\alpha, \mathrm{K}_{3}\right) \geq \begin{cases}0, & 0<\alpha \leq \frac{5}{16} \\ \frac{1}{8}\left(\alpha-\frac{5}{16}\right), & \frac{5}{16}<\alpha \leq \frac{3}{8} \\ \frac{1}{64}(4 \alpha-1), & \frac{3}{8}<\alpha \leq \frac{1}{2} \\ \frac{1}{64}(16 \alpha-7), & \frac{1}{2}<\alpha \leq \frac{5}{8} \\ \frac{3}{128}(8 \alpha-3), & \frac{5}{8}<\alpha \leq \frac{11}{16} \\ \frac{5}{32}(2 \alpha-1), & \frac{11}{16}<\alpha \leq 1\end{cases}
$$

\footnotetext{
${ }^{5} \mathrm{O}$ grafo $\mathrm{GHiSi}$ foi introduzido em 1956 por Mesner [Mes56] na sua tese de doutorado (não publicada). A unicidade para os parâmetros $(100,22,0,6)$ foi mostrada pelo mesmo autor em [Mes64]. Este grafo foi redescoberto (posterior e independentemente) por Higman e Sims [HS68] (veja [KW17] para uma detalhada revisão histórica).

${ }^{6}$ Cf. Brinkmann [Bri98] ou Brandt, Brinkmann e Harmuth [BBH98] onde também é usado o programa de computador mtf para a geração de grafos livres de triângulo aresta maximais.
} 


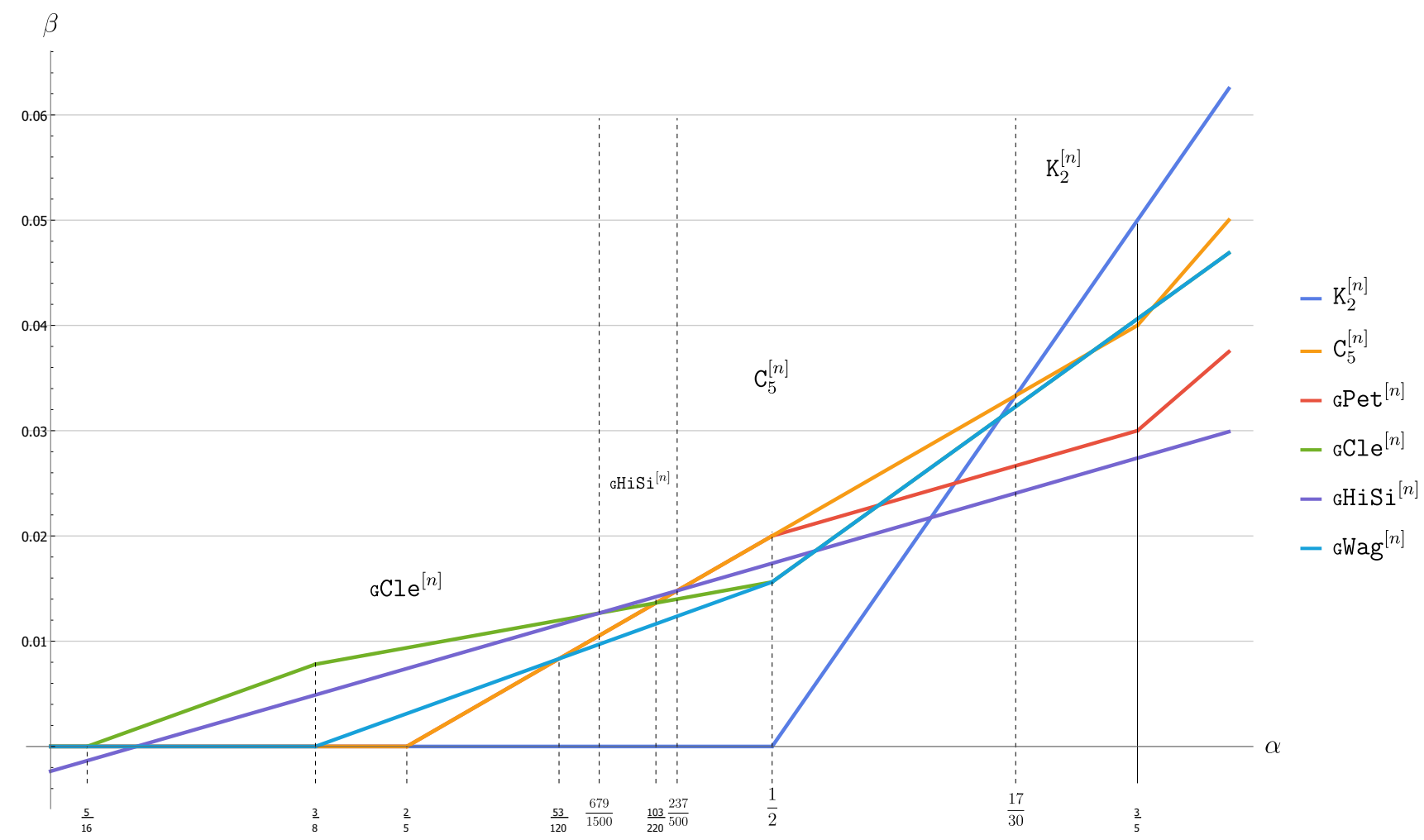

Figura 2: Melhor cota inferior para $\beta\left(\alpha, \mathrm{K}_{3}\right)$ conhecida nesta data

As constantes $\varepsilon$ e $\varepsilon^{\prime}$ são absolutas e facilmente calculáveis. Ao comparar a relação (4.1) no Teorema 1.8 com a relação (1.5) na Conjectura 1.7 temos que $\frac{1}{64}(4 \alpha-1)>\frac{5 \alpha-2}{25}$ para valores de $3 / 8<\alpha<103 / 220 \approx 0.468$, portanto, segue o seguinte corolário ao Teorema 1.8 .

Corolário 1.9. A Conjectura 1.7 é falsa no intervalo $\alpha \in\left[\frac{53}{120}, \frac{103}{220}\right)$.

\subsection{Notação}

Um grafo é um par ordenado $(V, E)$, onde $V$ é um conjunto de vértices e $E$ é um multiconjunto de arestas, cada uma sendo um par não ordenado de vértices em $V$ (não necessariamente distintos). Neste documento tenta-se uma exposição autocontida, no entanto, para uma ampla introdução à teoria de grafos, remetemos o leitor a qualquer um dos livros: Bollobás [Bol98], Bondy e Murty [BM11], Diestel [Die10] ou West [Wes05].

Nós seguimos a notação comumente usada na teoria de grafos (como em [Bol98, BM11, Die10, Wes05]) com poucas excepções. Se $A$ e $B$ são conjuntos e $k$ é um inteiro não negativo. Então $A \backslash B$ é a usual diferença de conjuntos entre $A$ e $B$. O inteiro $|A|$ é a cardinalidade de $A$. A família de subconjuntos de $A$ de cardinalidade $k$ é denotada $\left\{\begin{array}{l}A \\ k\end{array}\right\}$. Um $k$-subconjunto é um conjunto de cardinalidade $k$. O conjunto dos $k$ primeiros inteiros positivos é denotado $[k] \stackrel{\text { def }}{=}\{1, \ldots, k\}$. Para um grafo G, denotamos respectivamente $V(\mathrm{G})$ e $E(\mathrm{G})$ ao conjunto de vértices e o conjunto de arestas de G. O número de vértices $|\mathrm{G}| \stackrel{\text { def }}{=}|V(\mathrm{G})|$ do grafo G é a ordem de G. O número de arestas $e(\mathrm{G}) \stackrel{\text { def }}{=}|E(\mathrm{G})|$ é o tamanho de G. Por simplicidade, escrevemos uv para denotar o par não ordenado $\{u, v\}$. Dizemos que $u v$ é uma não aresta em $\mathrm{G}$ se $u v \notin E(\mathrm{G})$. Vértices $u, v \in V(\mathrm{G})$ são adjacentes se $u v \in E(\mathrm{G})$. Para um subconjunto $S \subseteq V(\mathrm{G})$ de vértices de G e um vértice $v \in V(\mathrm{G})$, a vizinhança de $v$ em $S$, denotada $N(v, S)$, é o conjunto de vértices adjacentes a $v \in V$ contidos em $S$, i.e.

$$
N(v, S) \stackrel{\text { def }}{=}\{u \in S: u v \in E(\mathrm{G})\}
$$


O inteiro $d(v, S) \stackrel{\text { def }}{=}|N(v, S)|$ é o grau de $v$ em $S$. Se $S=V(\mathrm{G})$ então escrevemos $N_{\mathrm{G}}(v)$ e $d_{\mathrm{G}}(v)$, ou simplesmente $N(v)$ e $d(v)$ se o grafo G é claro pelo contexto, em vez de $N(v, V(\mathrm{G}))$ e $d(v, V(\mathrm{G}))$. Neste caso, $N(v)$ e $d(v)$ são a vizinhança e o grau de $v$ em G respectivamente. O grau mínimo e máximo em G são denotamos, como usual e respectivamente, $\delta(\mathrm{G}) \stackrel{\text { def }}{=} \min \{d(v): v \in V(\mathrm{G})\}$ e $\Delta(\mathrm{G}) \stackrel{\text { def }}{=} \max \{d(v): v \in V(\mathrm{G})\}$.

Os ciclos com três e cinco vértices são chamados, triângulo e pentágono, respectivamente.

Seja $\mathrm{G}=(V, E)$ um grafo. O grafo complemento de $\mathrm{G}$ é o grafo $\mathrm{G}^{\mathrm{C}} \stackrel{\text { def }}{=}\left(V,\left\{\begin{array}{l}V \\ 2\end{array}\right\} \backslash E\right)$. Um grafo H é um subgrafo de G se $V(\mathrm{H}) \subseteq V(\mathrm{G})$ e $E(\mathrm{H}) \subseteq E(\mathrm{G})$; neste caso escrevemos $\mathrm{H} \subseteq \mathrm{G}$ ou $\mathrm{G} \supseteq \mathrm{H}$. Um conjunto independente em $\mathrm{G}$ é um conjunto de vértices de $\mathrm{G}$ no qual não existem dois vértices adjacentes. Como usual, $\alpha(\mathrm{G})$ é o número de independência de G, i.e., o maior tamanho de um conjunto independente em G. Uma $k$-coloração própria dos vértices de G é uma partição de $V(\mathrm{G})$ em $k$ conjuntos independentes. $\mathrm{O}$ menor inteiro $k$ para o qual $\mathrm{G}$ admite uma $k$-coloração própria é o número cromático $\chi(\mathrm{G})$ de $\mathrm{G}$. Um grafo $\mathrm{G}=(V, E)$ é finito se $V=V(\mathrm{G})$ é um conjunto finito, e G é simples se $E=E(\mathrm{G})$ é um conjunto de arestas cada uma sendo um par não ordenado de vértices distintos. Assim, grafos simples não contêm arestas múltiplas nem laços. A menos que especifiquemos o contrário, todo grafo considerado neste documento é finito e simples.

Seja H um grafo. Para um vetor de inteiros positivos $\eta \in \mathbb{Z}_{+}^{V(\mathrm{H})}$ o $\eta$-blow-up de H, é o grafo $\mathrm{H}^{[\eta]}$ obtido ao substituir cada vértice $v$ em $\mathrm{H}$ por um conjunto independente $I(v)$ de tamanho $\eta(v)$, e cada aresta $u v \in E(\mathrm{H})$ por um grafo bipartido completo com partições $I(u)$ e $I(v)$. Se $|\eta(u)-\eta(v)| \leq 1$ para cada $u, v \in V(\mathrm{H})$, o $\eta$-blow-up $\mathrm{H}^{[\eta]}$ é balanceado. Observe que para uma permutação $\eta^{\prime}$ de $\eta$, os grafos $\mathrm{H}^{\left[\eta^{\prime}\right]}$ e $\mathrm{H}^{[\eta]}$ não são em geral isomorfos (e.g. com $\eta=(2,2,2,1,1)$ e $\left.\eta^{\prime}=(2,2,1,2,1), \mathrm{C}_{5}{ }^{\left[\eta^{\prime}\right]} \not \mathrm{C}_{5}{ }^{[\eta]}\right)$, porém, nós escrevemos $\mathrm{H}^{[n]} \operatorname{com} n=\sum_{v \in V(\mathrm{H})} \eta(v)$ sempre que H é completo ou $\eta(u)=\eta(v)$ para todo $u, v \in V(\mathrm{H})$. Por exemplo, o grafo de Turán $\mathrm{T}_{n, r}$ é isomorfo a $\mathrm{K}_{r}^{[n]}$ para todo $n \geq r$.

\subsection{Extensões e problemas relacionados}

\subsubsection{Problemas tipo Turán-Ramsey}

O problema da densidade local em grafos pertence a uma categoria de problemas extremais conhecidos como problemas tipo Turán-Ramsey. Apesar da nossa definição de grafo em termos de conjuntos, na prática, no entanto, não é conveniente reduzir a teoria de grafos ao estudo clássico da teoria de conjuntos ou relações binarias. ${ }^{7}$ Isto é porque a teoria de grafos (e em particular a teoria de grafos extremais) está "cheia" de conjecturas e problemas onde tipicamente estamos interessados nas propriedades estruturais e extremais de uma família de grafos que satisfazem certa propriedade invariante por isomorfismos (dois grafos são isomorfos se existe uma rotulagem dos vértices tais que seus respectivos conjuntos de vértices e arestas são idênticos). Por exemplo, fixado um grafo H, determinar para cada inteiro $n$ o máximo número de arestas ex $(n, \mathrm{H})$ em grafos de $n$ vértices que não contêm uma copia de H. Nós chamamos estes grafos H-livre. Grafos H-livre de ordem $n$ com exatamente $\operatorname{ex}(n, \mathrm{H})$ arestas são os grafos extremais para este problema.

Um célebre resultado da teoria de grafos extremais é o teorema de Turán [Tur41] o qual estabelece que ex $\left(n, \mathrm{~K}_{r+1}\right)$ é igual ao número de arestas do grafo $\mathrm{T}_{n, r}$ (o grafo de Turán $\mathrm{T}_{n, r}$ é o grafo $r$-partido completo de ordem $n$ com partições que diferem em tamanho no máximo um vértice), sendo este grafo o único extremal a menos de isomorfismos. O caso particular para triângulos foi

\footnotetext{
${ }^{7}$ Este enfoque pragmático é análogo a como a teoria da probabilidade é considerada, num sentido informal, "mais que" o estudo de um espaço de medida com medida total 1 (para uma discussão, v. e.g. Tao [Tao12, p. 2]). No entanto, todo resultado sobre grafos fornece um resultado sobre conjuntos: um teorema de Szpilrajn-Marczewski [SM45] garante que a cada grafo G (finito ou infinito) corresponde-lhe uma família de conjuntos $\mathcal{F}(\mathrm{G})$ com um e só um conjunto por cada vértice em G, tais que dois vértices estão conectados por uma aresta em G se e só se os correspondentes conjuntos em $\mathcal{F}(\mathrm{G})$ são disjuntos.
} 
estabelecido previamente por Mantel em [Man07], i.e. ex $\left(n, \mathrm{~K}_{3}\right)=\left\lfloor\frac{n}{2}\right\rfloor\left\lceil\frac{n}{2}\right\rceil$, com o grafo bipartido completo de $n$ vértices de partições balanceadas o único extremal. Para algumas instancias deste e outros problemas, no entanto, não sempre é possível determinar os grafos extremais; nestes casos, resultados assintóticos são de interesse.

O número de Ramsey $R_{r}\left(s_{1}, s_{2}, \ldots, s_{k}\right)$ é o menor inteiro $n$ tal que para toda $k$-partição $E_{1} \cup$ $E_{2} \cup \cdots \cup E_{k}$ dos $r$-subconjuntos de $[n]$, existe um índice $1 \leq i \leq k$ para o qual $E_{i}$ contém os $r$-subconjuntos de um conjunto $S \subset[n]$ de tamanho $|S|=s_{i}$. Se $r=2$ (números de Ramsey para grafos), nós omitimos o subíndice. Ramsey [Ram29] mostrou que estes números existem para todos os valores dos parâmetros.

O teorema de Turán (1940) junto com o estudo dos números de Ramsey (1929) motivou o inicio de uma copiosa linha de pesquisa em combinatória extremal. ${ }^{8} \mathrm{O}$ paradigma comum que subjaz na teoria Turán-Ramsey ( cf. teoria Turán-Ramsey [SS01]) vem do mais básico principio de Dirichlet o qual "captura" o conceito geral de saturação num tipo particular de estrutura discreta. O principio de Dirichlet (1834) estabelece que toda $k$ partição de um conjunto de $k n+1$ elementos ( $k$ e $n$ inteiros), contém uma classe de cardinalidade pelo menos $n+1$. O resultado de Ramsey é de natureza análoga; fixados um inteiro $k$ e um grafo $\mathrm{H}$, o teorema de Ramsey garante que toda $k$-partição das $\left(\begin{array}{c}n \\ 2\end{array}\right)$ arestas do grafo completo $\mathrm{K}_{n}$ contém pelo menos uma classe "saturada" se $n$ é suficientemente grande. Com "saturada" queremos dizer que não consegue evitar uma copia de H. Um problema de interesse em combinatória extremal é determinar o menor inteiro $n$ para o qual o sistema sempre satura-se. Em contraste, em problemas tipo Turán fixa-se o número de vértices $n$ e um grafo $\mathrm{H}$ e procura-se o menor número de arestas para o qual todo grafo de $n$ vértices é necessariamente saturado. O teorema de Turán estabelece em particular que este valor é $e\left(\mathrm{~T}_{n, r}\right)+1$ para $\mathrm{H}=\mathrm{K}_{r+1}$.

\subsubsection{O Teorema de Turán}

O problema da densidade local em grafos visa estender o teorema de Turán. Como mencionado antes, em [Tur41], Turán mostrou que o único grafo que maximiza o número de arestas na família de grafos de $n$ vértices que não contêm $\mathrm{K}_{r+1}$ como subgrafo, é o grafo $r$-partido completo de $n$ vértices $\mathrm{T}_{n, r}$ com partições de tamanho $\left\lfloor\frac{n}{r}\right\rfloor,\left\lfloor\frac{n+1}{r}\right\rfloor, \ldots,\left\lfloor\frac{n+r-1}{r}\right\rfloor$. Para grafos livres de triângulo, este resultado foi estabelecido previamente por Mantel [Man07]. Em particular, todo grafo G de $n$ vértices com mais de $\left\lfloor\frac{n}{2}\right\rfloor\left\lceil\frac{n}{2}\right\rceil$ arestas contém um triângulo. Erdős perguntou qual é o menor número de arestas em subgrafos induzidos de G de $\left\lfloor\frac{n}{2}\right\rfloor$ vértices que implique a existência de um triângulo em G. Considerando o blow-up balanceado do pentágono, Erdős [Erd76] conjecturou que todo grafo G, no qual os subconjuntos de $\left\lfloor\frac{n}{2}\right\rfloor$ vértices induzem mais de $n^{2} / 50$ arestas, contém necessariamente um triângulo.

A seguinte questão é uma extensão natural à pergunta de Erdős. Fixado um real $\alpha \in(0,1]$ e um grafo H, quais são as condições sobre o número de arestas induzidas por subconjuntos de $\lfloor\alpha n\rfloor$ vértices num grafo $\mathrm{G}$ de ordem $n$ que impliquem a existência de H como subgrafo de G? Nós chamamos ao problema de determinar estas condições o Problema da Densidade Local em Grafos.

Problema 1.10 (O problema da densidade local em grafos). Para um real $\alpha \in(0,1]$ e um grafo $\mathrm{H}$, determine o menor real $\beta=\beta(\alpha, \mathrm{H})$ que satisfaz a seguinte propriedade: Se G é um grafo de $n$ vértices no qual cada subconjunto de $\lfloor\alpha n\rfloor$ vértices induz mais que $\beta n^{2}$ arestas então $\mathrm{G}$ contém $\mathrm{H}$ como subgrafo.

\footnotetext{
${ }^{8}$ Uma exposição de problemas e conjecturas em teoria de grafos extremais são dadas em Bollobás [Bol04] e Chung e Graham [CG99]. Para problemas e resultados tipo Ramsey veja Graham, Rothschild e Spencer [GRS13] e Xu, Liang e Luo [XLL18], ou Sudakov [Sud11] para um resumo de métodos e resultados. Importantes técnicas de solução de problemas em combinatória extremal estão baseados em ideias da teoria da probabilidade (v. e.g. [AS92, Spe94]), álgebra linear e análise espectral (v. e.g. [BF92, CDS95, GR01]), topologia e análise harmônica, entre outros (cf. [Sud11]).
} 
Em particular, a Conjectura 1.2 visa estabelecer $\beta\left(1 / 2, \mathrm{~K}_{3}\right)=1 / 50$, e de ser verdade, $\mathrm{C}_{5}^{[n]} \mathrm{e}$ GPet $^{[n]}$ são famílias de grafos extremais. Por outro lado, como $e\left(\mathrm{~T}_{n, r}\right)=\left(1-\frac{1}{r}+o(1)\right)\left(\begin{array}{l}n \\ 2\end{array}\right)$ (cf. [Tur54]) o teorema de Turán implica $\beta\left(1, \mathrm{~K}_{r+1}\right)=\frac{r-1}{2 r}$ com $\mathrm{T}_{n, r}$ o único grafo extremal. É fácil notar que se $1-\frac{1}{r}<\alpha \leq 1$ então o grafo $\mathrm{T}_{n, r}$ fornece a cota inferior

$$
\beta\left(1, \mathrm{~K}_{r+1}\right) \geq \frac{(r-1)(2 \alpha-1)}{2 r} .
$$

Keevash e Sudakov [KS03] mostraram que a igualdade na relação (1.8) vale sempre que $1-\frac{1}{2 r^{2}} \leq$ $\alpha \leq 1$, sendo o grafo de Turán $\mathrm{T}_{n, r}$ o único extremal. Eles conjecturaram que este resultado continua valendo ainda para $1-\frac{1}{r} \leq \alpha \leq 1$. Chung e Graham [CG90] conjecturaram que, exceto no caso $r=2$, o grafo de Turán $\mathrm{T}_{n, r}$ é extremal para $\beta\left(\alpha, \mathrm{K}_{r+1}\right)$ para todo $1 / 2 \leq \alpha \leq 1$. Isto é, que a cota inferior para $\beta\left(\alpha, \mathrm{K}_{r+1}\right)$ fornecida pelo grafo $\mathrm{T}_{n, r}$ é também cota superior para todo $1 / 2 \leq \alpha \leq 1 \mathrm{e}$ $r \geq 3$. Esta conjectura visa estabelecer, em particular, a igualdade

$$
\beta\left(1 / 2, \mathrm{~K}_{r+1}\right)=\left\{\begin{array}{ll}
\frac{r-2}{8 r}, & r \geq 3 \text { é par } \\
\frac{(r-1)^{2}}{8 r^{2}}, & r \geq 3 \text { é ímpar }
\end{array} .\right.
$$

Uma formulação mais geral do problema da densidade local para grafos (ou hipergrafos) é possível. Fixados um real $\alpha \in(0,1]$ e uma família de grafos proibidos $\mathcal{H}=\left\{\mathrm{H}_{1}, \ldots, \mathrm{H}_{t}\right\}$, determine ou estime para cada $n \in \mathbb{N}$ o menor inteiro $m(n ; \alpha, \mathcal{H})$ com a seguinte propriedade: se $\mathrm{G}$ é um grafo de $n$ vértices tal que todo subconjunto de $\lfloor\alpha n\rfloor$ vértices induz mais de $m(n ; \alpha, \mathcal{H})$ arestas, então G contém como subgrafo algum grafo na família $\mathcal{H}$. Por exemplo, do teorema de Turán segue $m\left(n ; 1, \mathrm{~K}_{r+1}\right)=e\left(\mathrm{~T}_{n, r}\right)$. O inteiro $m(n ; \alpha, \mathcal{H})$ tem a propriedade que todo grafo de $n$ vértices $\mathcal{H}$-livre contém um subconjunto de $\lfloor\alpha n\rfloor$ vértices que induz no máximo $m(n ; \alpha, \mathcal{H})$ arestas. A determinação de $m(n ; \alpha, \mathcal{H})$ é em geral um problema mais difícil que o Problema 1.10; em contraste, observe que no Problema 1.10 nós estamos apenas procurando um resultado assintótico para o quociente $m(n ; \alpha, \mathrm{H}) / n^{2}$, i.e. buscamos estimar a função

$$
\beta(\alpha, \mathrm{H}) \stackrel{\text { def }}{=} \limsup _{n \rightarrow \infty} \max _{\mathbf{G}_{n} \not \supset \mathrm{H}}\left\{\frac{e_{\alpha}\left(\mathrm{G}_{n}\right)}{n^{2}}\right\},
$$

onde o máximo na equação (1.9) é tomado sobre grafos $\mathrm{G}_{n}$ de ordem $n$ livres de $\mathrm{H}$. Este documento foca-se no caso $(\alpha, \mathrm{H})=\left(\frac{1}{2}, \mathrm{~K}_{3}\right)$ e os resultados principais mostram estimativas da função $\beta\left(\frac{1}{2}, \mathrm{~K}_{3}\right)$ quando enfraquecemos a Conjectura 1.2 impondo sobre o grafo livre de triângulo $\mathrm{G}$ alguma propriedade adicional (veja Seção 1.1 para um resumo de resultados).

\subsubsection{Número de pentágonos e diferença com o subgrafo bipartido máximo}

Erdős, Kleitman e Rothschild [EKR76] mostraram que quase todos os grafos livres de triângulo são bipartidos, i.e., se $\mathcal{T}_{n}$ e $\mathcal{B}_{n}$ são, respectivamente, o número de grafos livres de triângulo e o número de grafos bipartidos de $n$ vértices, então

$$
\mathcal{T}_{n}=(1+o(1)) \mathcal{B}_{n}
$$

Por outro lado, a existência de grafos livres de triângulo não bipartidos é bem conhecida. O pentágono, o grafo de Petersen e o grafo de Wagner, são exemplos de grafos livres de triângulo 3cromáticos. O grafo de Grötzsch e o grafo de Clebsch são grafos livres de triângulo 4-cromáticos (estes grafos são referenciados com muita frequência em todo o documento, cf. Figura 3).

Zykov [Zyk49] e Tutte [Des48] (cf. [Erd62]) mostraram a existência de grafos livres de triângulo $k$-cromáticos para todo inteiro positivo $k$ e Mycielski [Myc55] achou uma construção explícita de grafos com esta propriedade. Em [KK54] (cf. [Erd64]) Kelly e Kelly provaram que para todo inteiro 

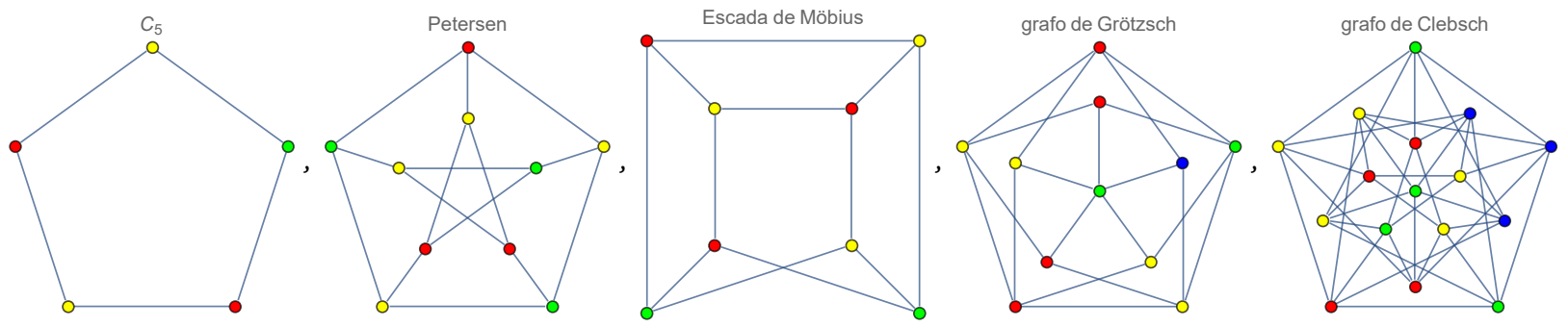

Figura 3: Exemplos de grafos livres de triângulo não bipartidos

positivo $k$ existem grafos $\left\{\mathrm{C}_{3}, \mathrm{C}_{4}\right\}$-livres $k$-cromáticos, e mais tarde, num célebre teorema usando métodos probabilísticos, Erdős [Erd59] estendeu este resultado ao mostrar a existência de grafos com cintura e número cromático arbitrários, i.e., para qualquer inteiro $k$ existe um grafo $\mathrm{G}$ com girtf $(\mathrm{G})>k$ e $\chi(\mathrm{G})>k$. A cintura girth $(\mathrm{G})$ de um grafo $\mathrm{G}$ é o tamanho do menor circuito em $\mathrm{G}$ (definida girth $(G)=\infty$ se G não contém circuitos).

Algumas conjecturas de Erdős sugerem ${ }^{9}$ que o blow-up balanceado do pentágono $\mathrm{C}_{5}^{[n]}$ tem propriedades que fazem dele um grafo livre de triângulo "longe" de ser bipartido. Erdös conjecturou (cf. e.g. [Erd71, Problem 2], [Erd76, Problems 5, 25] ou [Erd84, Problems 1, 2]) que, na família de grafos livres de triângulo, o grafo $\mathrm{G}=\mathrm{C}_{5}^{[n]}$ maximiza o valor de $\beta_{i},(i=1,2,3)$, em cada uma das seguintes propriedades

P1 Um grafo G de ordem $n$ livre de triângulo pode ser tornado bipartido pela remoção de no máximo $\beta_{1} n^{2}$ arestas.

P2 Um grafo G de ordem $n$ livre de triângulo contém um subconjunto de $\lfloor n / 2\rfloor$ vértices que induz no máximo $\beta_{2} n^{2}$ arestas.

P3 Um grafo G de ordem $n$ livre de triângulo contém no máximo $\beta_{3} n^{5}$ pentágonos.

É fácil ver que se G é bipartido então cada propriedade é satisfeita com $\beta_{i}=0$. Assim, procurar grafos extremais em cada propriedade (i.e. grafos G livres de triângulo que maximizem $\beta_{i}$ ) está relacionado com determinar "uma medida" de quão longe um grafo livre de triângulo está de ser bipartido. Se $5 \mid n$ então $C_{5}^{[n]}$ contém exatamente $\left(\frac{n}{5}\right)^{5}$ pentágonos e o menor número de arestas que precisamos remover para virar-lhe bipartido é exatamente $n^{2} / 25$. Se $10 \mid n$ então o menor número de arestas induzidas por subconjuntos de $n / 2$ vértices em $\mathrm{C}_{5}^{[n]}$ é exatamente $n^{2} / 50$.

Conjectura 1.11 (Erdős, 1971 [Erd71, Problem 2]). Todo grafo livre de triângulo de ordem n pode ser tornado bipartido pela remoção de no máximo $n^{2} / 25$ arestas.

Na data, a Conjectura 1.11 permanece aberta.

Para o número de pentágonos, o menor valor possível para $\beta_{3}$ que satisfaz a propriedade P3 foi estabelecido assintoticamente por Grzesik [Grz12] e independentemente por Hatami, Hladký, Král' e Razborov $\left[\mathrm{HHK}^{+} 13\right]$. Eles provaram que todo grafo livre de triângulo de ordem $n$ contém no máximo $\frac{5 !}{5^{5}}\left(\begin{array}{l}n \\ 5\end{array}\right)(1+o(1))$ pentágonos. O resultado de Hatami et al. [HHK $\left.{ }^{+} 13\right]$ prova além que se $5 \mid n$ ou $n$ é suficientemente grande, então $\mathrm{C}_{5}^{[n]}$ é o único grafo extremal. Michael [Mic13] observou que o grafo de Wagner (isomorfo à escada de Möbius de ordem 8 GMöb 8 ou ao grafo de Andrásfai $\Gamma_{3}$ ) contém o mesmo número de pentágonos que qualquer blow-up balanceado do $C_{5}$ sobre 8 vértices. Recentemente, Lidický e Pfender [LP17] resolveram o problema para todo $n$.

\footnotetext{
${ }^{9}$ Altamente subjectivo
} 
Teorema 1.12 (Lidický e Pfender [LP17]). Para todo n, o número máximo de pentágonos em qualquer grafo livre de triângulo de ordem $n$ é

$$
\prod_{i=0}^{4}\left\lfloor\frac{n+i}{5}\right\rfloor .
$$

Além disso, para todo $n \geq 5$ os únicos grafos extremais são blow-ups balanceados de $\mathrm{C}_{5}$ de ordem $n$, exceto no caso $n=8$ onde também aMöb 8 é extremal.

Erdős, Győri e Simonovits [EGS92] mostraram que grafos livres de triângulo com pelo menos $n^{2} / 5$ arestas satisfazem a Conjectura 1.11. Em [EFPS88] Erdős, Faudree, Pach e Spencer forneceram o melhor resultado conhecido na data para o caso geral.

Teorema 1.13 (Erdős, Faudree, Pach e Spencer [EFPS88]). Todo grafo livre de triângulo pode ser tornado bipartido pela remoção de no máximo $n^{2} / 18+n / 2$ arestas.

Teorema 1.14 (Erdős, Faudree, Pach e Spencer [EFPS88]). Existe uma constante $\varepsilon>0$ tal que todo grafo livre de triângulo de ordem $n$ pode ser tornado bipartido pela remoção de no máximo $\left(\frac{1}{18}-\varepsilon+o(1)\right) n^{2}$ arestas.

O problema da densidade local para grafos livres de triângulo tem sido estudado por vários autores [EFRS94, Kri95, Bra98, KS03, KS06, NY13, BMRS17] e importantes contribuições foram feitas. Referenciamos estes resultados ao longo do documento.

\subsubsection{Max-Cut e partições judiciosas}

Dado um grafo $\mathrm{G}=(V, E)$, o Problema do Subgrafo Bipartido Máximo pergunta por uma partição $V=V_{1} \cup V_{2}$ dos vértices de G que maximize o número de arestas de cruzamento $e\left(V_{1}, V_{2}\right)$. Este problema é NP-difícil [Kar72] ainda em grafos com grau máximo $\Delta(G) \leq 3$ [Yan78]. Para um grafo G, nós definimos

$$
\max \operatorname{Cut}(\mathrm{G}) \stackrel{\text { def }}{=} \max \{e(U, V \backslash U): U \subset V(\mathrm{G})\},
$$

como o máximo número de arestas num subgrafo bipartido de G. Certamente, maximizar $e\left(V_{1}, V_{2}\right)$ sobre partições $V_{1} \cup V_{2}$ é equivalente a minimizar $e\left(V_{1}\right)+e\left(V_{2}\right)$, assim

$$
\max C u t(\mathrm{G})=e(\mathrm{G})-\min \{e(U)+e(V \backslash U): U \subset V(\mathrm{G})\} .
$$

Para um inteiro $m>0$ nós definimos $\operatorname{maxCut}(m)$ como o menor valor de maxCut(G) para grafos G com $m$ arestas. Erdős observou que a cota $\operatorname{maxCut}(m) \geq m / 2$ pode ser obtida facilmente ao considerar uma bipartição aleatória de $V(\mathrm{G})$. Para notar isto, escolha cada vértice em $V(G)$ com probabilidade $1 / 2$. Se $U \subset V(G)$ é o subconjunto de vértices escolhidos, então a probabilidade de que cada aresta tenha um extremo em $U$ e o outro em $V \backslash U$ é $1 / 2$; portanto, o valor esperado da variável aleatória $e(U, V \backslash U)$ é $m / 2$, assim, existe uma partição com a propriedade requerida.

Edwards [Edw73, Edw75] mostrou que todo grafo G com $m$ arestas admite uma bipartição com

$$
\operatorname{maxCut}(\mathrm{G}) \geq \frac{m}{2}+\frac{1}{4}\left(\sqrt{2 m+\frac{1}{4}}-\frac{1}{2}\right) .
$$

Este resultado é justo para grafos completos de ordem ímpar. Provas mais simples do resultado de Edwards foram dadas por Erdős, Gyárfás e Kohayakawa [EGK97], Hofmeister e Lefmann [HL98], Lehel e Tuza [LT82] e Locke [Loc82].

Uma generalização do resultado de Edwards para $k$-partições, para todo $k \geq 2$, foi dada por Bollobás e Scott [BS02]. Eles provaram que todo grafo G com $m$ arestas admite uma $k$-partição 
$V_{1} \cup \cdots \cup V_{k}$ que satisfaz

$$
m-\sum_{i=1}^{k} e\left(V_{i}\right) \geq \frac{k-1}{k} m+\frac{k-1}{2 k}\left(\sqrt{2 m+\frac{1}{4}}-\frac{1}{2}\right)+O(k) .
$$

Nos problemas de partições judiciosas tenta-se otimizar várias quantidades simultaneamente. Para um inteiro $k$, nós chamamos uma $k$-partição de $V(\mathrm{G})=V_{1} \cup \ldots \cup V_{k}$ judiciosa se minimiza o inteiro $\max \left\{e\left(V_{i}\right): 1 \leq i \leq k\right\}$ entre todas as $k$-partições possíveis de $V(G)$. O caso $k=2$ é conhecido como o Problema da Bipartição do Gargalo da Garrafa ${ }^{10}$ (cf. Bottleneck Bipartition Problem). Shahrokhi e Székely [SS94] mostraram que este problema é NP-difícil.

Erdős (1988) conjecturou que todo grafo G com $m$ arestas admite uma bipartição $V(\mathrm{G})=V_{1} \cup V_{2}$ tal que

$$
\max \left\{e\left(V_{1}\right), e\left(V_{2}\right)\right\} \leq m / 4+o(\sqrt{m}) .
$$

Porter [Por92] mostrou que G admite uma bipartição $V(\mathrm{G})=V_{1} \cup V_{2}$ tal que

$$
\max \left\{e\left(V_{1}\right), e\left(V_{2}\right)\right\} \leq \frac{1}{4} m+\frac{1}{4} \sqrt{2 m} .
$$

Bollobás e Scott [BS99] melhoraram e estenderam o resultado de Porter ao provar que para todo $k \geq 2, \mathrm{G}$ admite uma $k$-partição $V_{1} \cup \ldots \cup V_{k}$ tal que

$$
\max _{1 \leq i \leq k}\left\{e\left(V_{i}\right)\right\} \leq \frac{1}{k^{2}} m+\frac{k-1}{2 k^{2}}\left(\sqrt{2 m+\frac{1}{4}}-\frac{1}{2}\right),
$$

esta cota é justa para todo $k$ e infinitos valores de $m$, e o grafo completo $\mathrm{K}_{k n+1}$ é o único grafo extremal sem vértices isolados ( $n$ um inteiro positivo). Além disso, para $k=2$ eles provaram que G admite uma bipartição $V(\mathrm{G})=V_{1} \cup V_{2}$ que satisfaz simultaneamente a cota (1.10) e também a cota de Edwards.

Alon [Alo96] mostrou que se G é livre de triângulo então maxCut $(\mathrm{G}) \geq \frac{m}{2}+\mathrm{cm}^{4 / 5}$, para alguma constante $c>0$ e este resultado é justo salvo pelo fator constante. Alon, Bollobás, Krivelevich e Sudakov [ABKS03] estenderam este resultado para grafos com cintura $r \geq 3$ e forneceram, entre outros, dois resultados para bipartições judiciosas em função do corte bipartido máximo. Eles mostraram que se $\mathrm{G}=(V, E)$ é um grafo com $m$ arestas com corte bipartido máximo maxCut $(\mathrm{G})=$ $\frac{m}{2}+\theta$. Então, se $\theta \leq m / 30$, então existe uma partição $V=V_{1} \cup V_{2}$ dos vértices de G tal que

$$
e\left(V_{i}\right) \leq \frac{m}{4}-\frac{\theta}{2}+\frac{10 \theta^{2}}{m}+3 \sqrt{m}, i=1,2,
$$

e se $\theta \geq m / 30$ e $m$ é suficientemente grande, então existe uma partição $V=V_{1} \cup V_{2}$ dos vértices de $G$ tal que

$$
e\left(V_{i}\right) \leq \frac{m}{4}-\frac{m}{100}=\frac{6 m}{25}, i=1,2 .
$$

${ }^{10}$ Tradução livre 


\section{Capítulo 2}

\section{Grafos livres de triângulos e pentágonos}

Erdős, Faudree, Rousseau \& Schelp [EFRS94] provaram que a Conjectura 1.2 vale se a constante $\frac{1}{50}$ é substituída por $\frac{\sqrt{65}-7}{32}(<1 / 30)$ e Krivelevich [Kri95] verificou a sua validez ao substituir $1 / 50$ por $1 / 36$ fornecendo até agora o melhor resultado nesta direção.

Teorema 2.1 (Krivelevich [Kri95]). Se, em um grafo G de ordem n, cada $\lfloor n / 2\rfloor$ vértices induzem pelo menos $n^{2} / 36$ arestas, então $\mathrm{G}$ contém um triângulo.

Como notamos antes, a constante $1 / 50$ na Conjectura 1.2 não pode ser substituída por uma constante menor uma vez que, para infinitos valores de $n$, existe um grafo G de $n$ vértices e livre de triângulo no qual, o mínimo sobre o número de arestas dos subgrafos induzidos por conjuntos de $\lfloor n / 2\rfloor$ vértices é exatamente $n^{2} / 50$. Além do grafo $C_{5}^{[10 n]}$, foi observado por Simonovits (cf. e.g. [Erd84] ou [Erd97]) que o grafo GPet $^{[10 n]}$ também atinge a constante 1/50 (veja Figura 1).

Além dos blow-ups do pentágono e do grafo de Petersen, nós não sabemos se existe outra família de grafos livres de triângulo que atinjam o valor conjecturado. Estes grafos tem cintura impar ogirth $(\mathrm{G})=5$. Claramente, um grafo $\mathrm{G}$ é livre de triângulo se e só se ogirth $(\mathrm{G}) \geq 5$. Nós provamos que se a desigualdade é estrita, não só a Conjectura 1.2 verifica-se como também a constante $1 / 50$ pode ser decrementada.

Teorema 1.3. Se G é um grafo de $n$ vértices e cintura ímpar ogirth(G) $>5$. Então, G contém $\lfloor n / 2\rfloor$ vértices que induzem no máximo $n^{2} / 64$ arestas.

Nós provamos este resultado na Seção 2.2.

Um homomorfismo de grafos $\mathrm{G} \rightarrow \mathrm{H}$ é um mapeamento $f: V(\mathrm{G}) \rightarrow V(\mathrm{H})$ tal que $u v \in E(\mathrm{G})$ implica $f(u) f(v) \in E(\mathrm{H})$. Assim, um homomorfismo $\mathrm{G} \rightarrow \mathrm{H}$ é um mapeamento de vértices de $\mathrm{G}$ em vértices de $\mathrm{H}$ que preserva as arestas. Por exemplo, $\mathrm{G}$ é $k$-colorível se e só se $\mathrm{G} \rightarrow \mathrm{K}_{k}$. Necessariamente, pré-imagens por homomorfismos de grafos $\mathrm{K}_{k}$-livres são grafos $\mathrm{K}_{k}$-livres.

Uma importante família de grafos livres de triângulo são os grafos de Andrásfai descritos em [And62]. ${ }^{1}$

Definição 2.2 (Grafos de Andrásfai). Para cada inteiro $i \geq 1$, o grafo de Andrásfai $\Gamma_{i}$ é o grafo com conjunto de vértices $V\left(\Gamma_{i}\right) \stackrel{\text { def }}{=}\{1, \ldots, 3 i-1\}$, com dois vértices $u, v \in V\left(\Gamma_{i}\right)$ sendo adjacentes se e só se $|u-v| \equiv 1 \bmod 3$.

Por exemplo, $\Gamma_{1}=\mathrm{K}_{2}, \Gamma_{2}=\mathrm{C}_{5}, \Gamma_{3}$ é a escada de Möbius de 4 degraus, etc. (v. Figura 4).

\footnotetext{
${ }^{1}$ A descrição em [And62] é provavelmente a primeira conhecida, porém estes grafos foram redescobertos várias vezes (cf. [Bra02, p. 36]). Para a definição original de Andrásfai em [And62, (em alemão)] veja por exemplo [GR01, $\S 6$. Exercise 39, p. 130].
} 

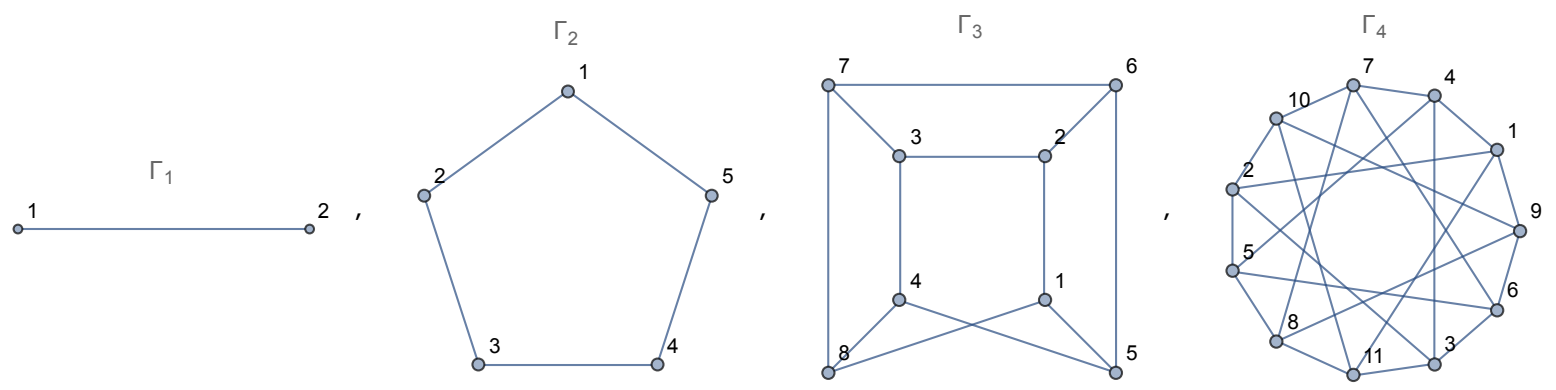

Figura 4: Grafos de Andrásfai $\Gamma_{i},(i=1 \ldots 4)$

$\mathrm{O}$ grafo $\Gamma_{i}$ é $i$-regular, não contém vértices similares (i.e., as vizinhanças de pares vértices são distintas), tem diâmetro 2 e é livre de triângulo (cf. e.g. [GR01, Lemma 6.10.1]). Desde que a adjacência de um vértice $j$ em $\Gamma_{i}$ é $N(j)=\{j+i, \ldots, j+2 i-1\}$ (tomando módulo $3 i-1$ em cada elemento), temos que $\Gamma_{i}$ é 3-colorível e

$$
V\left(\Gamma_{i}\right)=\{1, \ldots, i\} \cup\{i+1, \ldots, 2 i\} \cup\{2 i+1, \ldots, 3 i-1\}
$$

é uma 3-coloração de $\Gamma_{i}$ para todo $i \geq 2$.

Andrásfai, Erdős e Sós [AES74] provaram que em grafos livres de triângulo, a condição $\delta(G)>$ $2 n / 5$ implica $\mathrm{G} \rightarrow \Gamma_{1}$; Häggkvist [Häg82] mostrou que $\delta(\mathrm{G})>3 n / 8$ implica $\mathrm{G} \rightarrow \Gamma_{2}$, e finalmente Jin [Jin93] mostrou que $\delta(\mathrm{G})>\frac{i+1}{3 i+2} n$ implica $\mathrm{G} \rightarrow \Gamma_{i},(1 \leq i \leq 9)$. Assim, estes resultados garantem que grafos de Andrásfai são imagens por homomorfismos de grafos livres de triângulo com grau mínimo suficientemente grande. Em particular, $\delta(G)>10 n / 29$ implica $G \rightarrow \Gamma_{9}$ (sempre que G seja livre de triângulo).

Teorema 2.3 (Jin [Jin93]). Para $1 \leq i \leq 9$, todo grafo G livre de triângulo de ordem $n$ com grau mínimo $\delta(\mathrm{G})>\frac{i+1}{3 i+2}$ n é homomorfo $a \Gamma_{i}$.

O blow-up não balanceado do grafo de Grötzsch GGrö da Figura 7, um grafo 4-colorível 10regular de ordem 29, mostra que para $i=10$ a declaração do Teorema 2.3 falha.

Norine e Yepremyan [NY13] mostraram que grafos homomorfos ao grafo de Andrásfai $\Gamma_{i}$, para algum $2 \leq i \leq 4$ (o caso $i=1$ é trivial), satisfazem a Conjectura 1.2. Como corolário deste resultado e do Teorema 2.3, a condição $\delta(G)>5 n / 14$ implica que a Conjectura 1.2 se satisfaz.

Teorema 2.4 (Norine e Yepremyan [NY13]). Se G é homomorfo a algum grafo de Andrásfai $\Gamma_{i}$, com $2 \leq i \leq 4$. Então $\mathrm{G}$ contém um subconjunto de $\lfloor n / 2\rfloor$ vértices que induz no máximo $n^{2} / 50$ arestas.

Corolário 2.5 (Norine e Yepremyan [NY13]). Se G é um grafo livre de triângulo de ordem n com grau mínimo $\delta(\mathrm{G})>5 n / 14$ então $\mathrm{G}$ contém um subconjunto de $\lfloor n / 2\rfloor$ vértices que induz no máximo $n^{2} / 50$ arestas.

Recentemente, Bedenknecht, Mota, Reiher e Schacht [BMRS17] estenderam o Teorema 2.4. Eles provam que grafos homomorfos ao grafo de Andrásfai generalizado $\Gamma_{i, k}$ contém um subconjunto de $\lfloor n / 2\rfloor$ vértices que induz no máximo $n^{2} /\left(2(2 k+1)^{2}\right)$ arestas.

Definição 2.6 (Grafo de Andrásfai generalizado). Para inteiros $i \geq 1$ e $k \geq 2$, o grafo de Andrásfai generalizado $\Gamma_{i, k}$ é o grafo sobre o conjunto de vértices $\{1,2, \ldots,(2 k-1)(i-1)+2\}$ tal que a vizinhança de cada vértice $u \in V\left(\Gamma_{i, k}\right)$ é

$$
N(u) \stackrel{\text { def }}{=}\{v:(k-1)(i-1)+1 \leq|u-v| \leq k(i-1)+1\} .
$$


A $k$-ésima potência de um grafo $\mathrm{H}$ é o grafo $\mathrm{H}^{k}$ que resulta ao adicionar arestas em $\mathrm{H}$ entre pares de vértices (distintos) a distância no máximo $k$. Para $i \geq 2$, o grafo de Andrásfai $\Gamma_{i}$ é isomorfo ao grafo complemento da $(i-1)$-ésima potência do $(3 i-1)$-ciclo, i.e. $\Gamma_{i}=\mathrm{G}\left(\mathrm{C}_{3 i-1}^{i-1}\right)^{\mathrm{c}}$. Por outro lado, o grafo de Andrásfai generalizado $\Gamma_{i, k}$ é isomorfo ao grafo complemento da $(k-1)(i-1)$-ésima potência do $((2 k-1)(i-1)+2)$-ciclo, i.e. $\left.\Gamma_{i, k}=\mathrm{G}\left(\mathrm{C}_{(2 k-1)(i-1)+2}^{(k-1)(i-1)}\right)^{\mathrm{C}}(\mathrm{cf} \text {. [BRB09] }]^{2}\right)$.

Assim temos, $\Gamma_{1, k}=\mathrm{K}_{2}, \Gamma_{2, k}=\mathrm{C}_{2 k+1}, \Gamma_{3, k}=\mathrm{GMöb}_{4 k}$, e para $k=2$ temos os grafos de Andrásfai $\Gamma_{i, 2}=\Gamma_{i}$, etc. O grafo $\Gamma_{i, k}$ é $i$-regular e tem cintura ímpar ogirth $\left(\Gamma_{i, k}\right)=2 k+1$.

Teorema 2.7 (Bedenknecht, Mota, Reiher e Schacht [BMRS17]). Se G é homomorfo ao grafo de Andrásfai generalizado $\Gamma_{i, k},(i \geq 2, k \geq 1)$. Então $\mathrm{G}$ contém um subconjunto de $\lfloor n / 2\rfloor$ vértices que induz no máximo $n^{2} /\left(2(2 k+1)^{2}\right)$ arestas.

Albertson, Chan e Haas [ACH93] e Häggkvist e Jin [HJ98] mostraram que se G é um grafo de ordem $n$ com ogirth $(\mathrm{G})=7$ e $\delta(\mathrm{G})>n / 4$ então $\mathrm{G} \rightarrow \mathrm{C}_{7}$. Messuti e Schacht [MS14] e Brandt e Ribe-Baumann [BRB09, Theorem 2.4] provaram que grafos com cintura ímpar ogirth $(\mathrm{G})=2 k+1$, $(k \geq 2)$, e grau mínimo $\delta(\mathrm{G})>\frac{3}{4 k} n$ (veja [BRB09] para uma melhor cota no caso $k=3$ ) são homomorfos a $\mathrm{C}_{2 k+1}$. Recentemente, Letzter e Snyder [LS18], resolvendo um problema de Messuti e Schacht [MS14], mostraram que grafos com cintura ímpar ogirth $(G)>5$ e grau mínimo $\delta(G)>n / 5$ são homomorfos ao grafo de Andrásfai generalizado $\Gamma_{i, 3}$ para algum $i \geq 1$. Este resultado junto com o Teorema 2.7 implicam que se ogirth $(\mathrm{G})=2 k+1$, com $k \geq 3$, e $\delta(\mathrm{G})>n / 5$ então $\mathrm{G}$ contém um subconjunto de $\lfloor n / 2\rfloor$ vértices que induz no máximo $n^{2} /\left(2(2 k+1)^{2}\right)$ arestas. Em particular, para $k=3$, este resultado permite substituir a constante $1 / 64$ por $1 / 98$ no Teorema 1.3 sempre que a condição $\delta(\mathrm{G})>n / 5$ seja adicionada, ou equivalentemente, sempre que G seja um subgrafo de um blow-up (não necessariamente balanceado) de $\Gamma_{i, 3}$. Assim, embora nossa cota no Teorema 1.3 é mais fraca, esta é mais geral no sentido de que independe do homomorfismo ou da cota no grau mínimo.

\subsection{Corte em grafos $(\alpha, \beta)$-densos}

Definição 2.8. Para $\alpha, \beta \in(0,1]$, nós dizemos que um grafo $\mathbf{G}$ de ordem $n$ é $(\alpha, \beta)$-denso se

$$
e_{\alpha}(\mathrm{G})>\beta n^{2} .
$$

Lema 2.9. Seja $\mathrm{G}=(V, E)$ um grafo com $n$ vértices $(\alpha, \beta)$-denso. Sejam $X$ e $Y$ subconjuntos de vértices de $\mathrm{G}$ disjuntos e não vazios que satisfazem $|Y| \leq\lfloor\alpha n\rfloor-1$ e $|X|+|Y| \geq\lfloor\alpha n\rfloor$. Então

$$
e(X, Y)>\frac{|X|}{\lfloor\alpha n\rfloor-|Y|}\left(\beta n^{2}-e(Y)\right)-\frac{(\lfloor\alpha n\rfloor-|Y|)}{|X|} e(X) .
$$

Em particular, se $X$ e $Y$ são conjuntos independentes então $e(X, Y)>\beta|X| n^{2} /(\alpha n-|Y|)$.

Prova. Sejam $x=|X|, y=|Y|$ e $k=\lfloor\alpha n\rfloor-y$. Como $X$ e $Y$ são disjuntos, na soma $\sum_{W \in\left\{\begin{array}{l}X \\ k\end{array}\right\}} e(W \cup Y)$ cada aresta em $E(X)$ é contada $\left(\begin{array}{l}x-2 \\ k-2\end{array}\right)$ vezes e cada aresta em $E(X, Y)$ é contada

\footnotetext{
${ }^{2}$ Existe um pequeno erro tipográfico em [BRB09] no final da pág. 90, onde diz " $M_{j, k}$ is a $j$-regular graph of odd girth $k$ " deveria dizer " $M_{j, k}$ is a $j$-regular graph of odd girth $2 k+1$ ".
} 
$\left(\begin{array}{l}x-1 \\ k-1\end{array}\right)$ vezes. Portanto,

$$
\begin{aligned}
\sum_{W \in\left\{\begin{array}{l}
X \\
k
\end{array}\right\}} e(W \cup Y) & =\left(\begin{array}{l}
x-2 \\
k-2
\end{array}\right) e(X)+\left(\begin{array}{l}
x-1 \\
k-1
\end{array}\right) e(X, Y)+\left(\begin{array}{l}
x \\
k
\end{array}\right) e(Y) \\
& =\left(\begin{array}{l}
x \\
k
\end{array}\right)\left[\frac{k(k-1)}{x(x-1)} e(X)+\frac{k}{x} e(X, Y)+e(Y)\right] \\
& \leq\left(\begin{array}{l}
x \\
k
\end{array}\right)\left(\frac{k^{2}}{x^{2}} e(X)+\frac{k}{x} e(X, Y)+e(Y)\right) .
\end{aligned}
$$

Por outro lado, como $|W \cup Y|=\lfloor\alpha n\rfloor$ para cada $W \in\left\{\begin{array}{l}X \\ k\end{array}\right\}$, da $(\alpha, \beta)$-densidade de G segue

$$
\sum_{W \in\left\{\begin{array}{l}
X \\
k
\end{array}\right\}} e(W \cup Y)>\left(\begin{array}{l}
x \\
k
\end{array}\right) \beta n^{2} .
$$

De $(2.2)$ e $(2.3)$ temos

$$
\frac{k^{2}}{x^{2}} e(X)+\frac{k}{x} e(X, Y)+e(Y)>\beta n^{2}
$$

$\mathrm{Ou}$

$$
e(X, Y)>\frac{x}{k}\left(\beta n^{2}-e(Y)\right)-\frac{k}{x} e(X) .
$$

A desigualdade (2.1) segue ao substituir os valores de $x, y$ e $k$ em (2.4).

Nós também fazemos uso dos seguintes lemas.

Lema 2.10 (Krivelevich [Kri95, Lemma 2]). Se G é um grafo livre de triângulos de n vértices e $m$ arestas, então existem em $\mathrm{G}$ dois subconjuntos independentes disjuntos e não vazios $A$ e $B$ tais que $|A|+|B| \geq 4 m / n$.

Lema 2.11 ([Kri95, Lemma 1]). Seja G um grafo de $n$ vértices $(\alpha, \beta)$-denso. Suponha que $\gamma$ é um real tal que $\alpha \leq \gamma \leq 1$. Então, Todo subconjunto de $\lfloor\gamma n\rfloor$ vértices induz mais de $\frac{\beta \gamma^{2}}{\alpha^{2}} n^{2}$ arestas.

\subsection{Prova do Teorema $\mathbf{1 . 3}$}

Seja $\mathrm{G}=(V, E)$ um grafo de $n$ vértices com cintura ímpar ogirth $(\mathrm{G})>5$. Suponha pelo contrário que G é $(1 / 2,1 / 64)$-denso. Seja $\rho=e(\mathrm{G}) / n^{2}$. Então, a desigualdade de Cauchy-Schwarz implica que

$$
\sum_{u \in V} \sum_{w \in N(u)} d(w)=\sum_{u \in V} d^{2}(u) \geq \frac{1}{n}\left(\sum_{u \in V} d(u)\right)^{2}=4 \rho^{2} n^{3},
$$

portanto, existe um vértice $u_{0} \in V$ tal que

$$
\sum_{w \in N\left(u_{0}\right)} d(w) \geq 4 \rho^{2} n^{2}
$$

Seja $w_{0}$ um vértice em $N\left(u_{0}\right)$ e sejam

$$
U=\bigcup_{v \in N\left(u_{0}\right)} N(v)
$$

e

$$
W=\bigcup_{v \in N\left(w_{0}\right)} N(v)
$$


Afirmação. Afirmamos que $U$ e $W$ são conjuntos independentes disjuntos e não vazios tais que

$$
e(U, W) \geq 4 \rho^{2} n^{2} .
$$

Prova da Afirmação. Como $u_{0} \in N\left(w_{0}\right) \subseteq U$ e $w_{0} \in N\left(u_{0}\right) \subseteq W$ temos $U \neq \varnothing$ e $W \neq \varnothing$. Se existir $v \in U \cap W$ então existem vértices $x \in N\left(u_{0}\right)$ e $y \in N\left(w_{0}\right)$ adjacentes a $v$. Como G é livre de triângulo temos $N\left(u_{0}\right) \cap N\left(w_{0}\right)=\varnothing$ e assim $x \neq y$, mas agora, $x v y w_{0} u_{0}$ é um pentágono em G o que contradiz a condição ogirth $(\mathrm{G})>5$, logo tal $v$ não existe e assim $U$ e $W$ são disjuntos. Analogamente, suponha que $u_{1} u_{2}$ é uma aresta em G com ambos extremos em $U$. Da definição de $U$, existem vértices $w_{1}, w_{2} \in N\left(u_{0}\right)$ tais que $u_{1} w_{1}$ e $u_{2} w_{2}$ são arestas de $\mathrm{G}$, mas se $w_{1}=w_{2}$ (ou $u_{1}=u_{0}$ ou $u_{2}=u_{0}$ ) então $u_{1} w_{1} u_{2}$ é um triângulo em G, e se $w_{1} \neq w_{2}$ então $u_{0} w_{1} u_{1} u_{2} w_{2}$ é um pentágono em G, uma contradição a ogirth $(\mathrm{G})>5$. Isto implica que $U$ é um conjunto independente. Similarmente temos que $W$ é um conjunto independente. Agora como $W \supseteq N\left(u_{0}\right)$ de $(2.5)$ segue

$$
e(U, W) \geq e\left(U, N\left(u_{0}\right)\right)=\sum_{w \in N\left(u_{0}\right)} d(w) \geq 4 \rho^{2} n^{2}
$$

o que completa a prova da afirmação.

Seja $T$ o complemento de $U \cup W$ em $V(\mathrm{G})$. Sejam $x=|U| / n$ e $y=|W| / n$, assim $1-x-y=|T| / n$. Sejam $\rho_{1}=e(U, W) / n^{2}, \rho_{2}=e(U, T) / n^{2}, \rho_{3}=e(W, T) / n^{2}$ e $\rho_{4}=e(T) / n^{2}$. A $(1 / 2,1 / 64)$-densidade de G implica que $x<1 / 2$ e $y<1 / 2$, assim, podemos aplicar o Lema 2.9 aos conjuntos $U$ e $T$ como também aos conjuntos $W$ e $T$ para obter de (2.1) respectivamente

$$
\begin{aligned}
& \rho_{2}>\frac{1-x-y}{64(1 / 2-x)}-\frac{(1 / 2-x)}{1-x-y} \rho_{4} \\
& \rho_{3}>\frac{1-x-y}{64(1 / 2-y)}-\frac{(1 / 2-y)}{1-x-y} \rho_{4} .
\end{aligned}
$$

De $(2.7)$ e (2.8) obtemos

$$
\rho_{2}+\rho_{3}+\rho_{4}>\frac{1}{64}\left(\frac{1-x-y}{1 / 2-x}+\frac{1-x-y}{1 / 2-y}\right) .
$$

Por outro lado, na região $0<x<1 / 2,0<y<1 / 2$, a função $h(x, y)=\frac{1-x-y}{1 / 2-x}+\frac{1-x-y}{1 / 2-y}$ é minimizada nos pontos da reta $y=x$, portanto $h(x, y) \geq 4$. Como $\rho=\sum_{i=1}^{4} \rho_{i}$, então de (2.9) segue

$$
\rho-\rho_{1}>\frac{1}{16}
$$

Por (2.6) temos $\rho_{1} \geq 4 \rho^{2}, \operatorname{logo}$ de (2.10) segue $\rho>\frac{1}{16}+4 \rho^{2}$ ou

$$
(8 \rho-1)^{2}<0
$$

uma contradição, assim G não é $(1 / 2,1 / 64)$-denso como suposto, o que completa a prova.

\subsection{Grafos livres de triângulo com grau médio acotado}

Keevash e Sudakov [KS06] consideraram o inteiro $e(G):=|E(G)|$, o número de arestas de G. É fácil ver que a Conjectura 1.2 vale se $e(G) \leq 2 n^{2} / 25$. Se escolhemos uniformemente ao acaso um subconjunto $U \subset V(\mathrm{G})$ de tamanho $|U|=\lfloor n / 2\rfloor$ então para cada aresta $\{u, v\} \in E(\mathrm{G})$, a 
probabilidade de que ambos dos seus extremos pertençam a $U$ é

$$
\mathbb{P}[\{u, v\} \subset U]=\frac{\left(\begin{array}{c}
n-2 \\
\lfloor n / 2\rfloor-2
\end{array}\right)}{\left(\begin{array}{c}
n \\
\lfloor n / 2\rfloor
\end{array}\right)}=\frac{\lfloor n / 2\rfloor(\lfloor n / 2\rfloor-1)}{n(n-1)}<\frac{1}{4} .
$$

A linearidade do valor esperado fornece um $U$ de tamanho $\lfloor n / 2\rfloor$ tal que $e(U)<e(\mathrm{G}) / 4$, assim se $e(\mathrm{G}) \leq 2 n^{2} / 25$ então $e(U)<n^{2} / 50$.

Keevash e Sudakov [KS06] provaram que a Conjectura 1.2 vale sempre que $e(\mathrm{G}) \geq n^{2} / 5$ ou $e(G) \leq n^{2} / 12$. Nosso resultado melhora modestamente a cota superior no resultado de Keevash e Sudakov [KS06]. Nossos cálculos permitem trocar a constante $\frac{1}{12}$ pela constante $c_{1}=\frac{33-\sqrt{161}}{232} \approx$ $\frac{1}{11.422}$, no entanto, apresentamos o resultado com $c_{2}=\frac{7}{80} \approx \frac{1}{11.429}$.

Proposição 1.4. Seja G um grafo livre de triângulos de ordem $n$ com no máximo $\frac{7}{80} n^{2}$ arestas. Então G contém um subgrafo induzido de ordem $\lfloor n / 2\rfloor$ com no máximo $n^{2} / 50$ arestas.

Prova. Seja $c=(33-\sqrt{161}) / 232$. Vamos mostrar que todo grafo $G$ de ordem $n$ livre de triângulos com $e(G) \leq c n^{2}$ arestas satisfaz $e_{1 / 2}(G) \leq n^{2} / 50$. Como $c>\frac{7}{80}$, o resultado segue.

Para tanto, seja G um grafo livre de triângulos com $n$ vértices e $\rho n^{2}$ arestas para algum $\rho \leq c$. Suponha pelo contrário que G é $(1 / 2,1 / 50)$-denso. O Lema 2.10 garante que existem dois conjuntos independentes disjuntos e não vazios $A$ e $B$ tais que $|A|+|B|=4 \rho n$. Seja $C=V(\mathrm{G}) \backslash A \cup B$ o complemento em $V(\mathrm{G})$ de $A \cup B$. Como $A$ e $B$ são independentes, da $(1 / 2,1 / 50)$-densidade de G segue que $|A|<n / 2$ e $|B|<n / 2$. Dado que $\rho<1 / 8$ temos ainda que $|A \cup B|<n / 2$ e portanto $|A \cup C|>n / 2$ e $|B \cup C|>n / 2$. Assim, os conjuntos $A$ e $C$ satisfazem as condições do Lema 2.9 (com $X=C$ e $Y=A$ ). De igual modo, os conjuntos $B$ e $C$ (com $X=C$ e $Y=B$ ). Denotando $a=|A| / n, \rho_{1}=e(C) / n^{2}, \rho_{2}=e(A, C) / n^{2}, \rho_{3}=e(B, C) / n^{2}, \rho_{4}=e(A \cup B) / n^{2}$ e lembrando que $A$ e $B$ são conjuntos independentes, então do Lema 2.9 nós obtemos

$$
\begin{aligned}
\left(\frac{1 / 2-a}{1-4 \rho}\right)^{2} \rho_{1}+\left(\frac{1 / 2-a}{1-4 \rho}\right) \rho_{2} & >1 / 50, \\
\left(\frac{1 / 2-4 \rho+a}{1-4 \rho}\right)^{2} \rho_{1}+\left(\frac{1 / 2-4 \rho+a}{1-4 \rho}\right) \rho_{3} & >1 / 50, \\
\left(\frac{1 / 2-4 \rho}{1-4 \rho}\right)^{2} \rho_{1}+\left(\frac{1 / 2-4 \rho}{1-4 \rho}\right)\left(\rho_{2}+\rho_{3}\right)+\rho_{4} & >1 / 50 .
\end{aligned}
$$

Multiplicando (2.11) por $\frac{1-4 \rho}{1 / 2-a}$ e (2.12) por $\frac{1-4 \rho}{1 / 2-4 \rho+a}$ e somando obtemos

$$
\rho_{1}+\rho_{2}+\rho_{3}>\frac{1}{50}\left(\frac{1-4 \rho}{1 / 2-a}+\frac{1-4 \rho}{1 / 2-4 \rho+a}\right)
$$

Lembrando que $\rho<1 / 4$ temos que a função

$$
f(x)=\frac{1-4 \rho}{1 / 2-x}+\frac{1-4 \rho}{1 / 2-4 \rho+x}
$$

é minimizada em $x=2 \rho$, e claramente, $f(2 \rho)=4$. De isto e da igualdade $\rho=\sum_{i=1}^{4} \rho_{i}$, obtemos de (2.14) que

$$
\rho_{4}<\rho-\frac{2}{25} .
$$

Por outro lado, como $(1-4 \rho) n=|C|>n / 2$, do Lema 2.11 obtemos

$$
\rho_{1}>\frac{2}{25}(1-4 \rho)^{2}
$$


Fazendo $s=\frac{1 / 2-4 \rho}{1-4 \rho}$, podemos reescrever (2.13) como

$$
s^{2} \rho_{1}+s\left(\rho_{2}+\rho_{3}\right)+\rho_{4}>1 / 50
$$

ou equivalentemente

$$
s \sum_{i=1}^{4} \rho_{i}>\frac{1}{50}+\left(s \rho_{1}-\rho_{4}\right)(1-s)
$$

de $(2.15)$ e $(2.16)$ obtemos

$$
s \rho_{1}-\rho_{4}>\frac{1}{25}\left(32 \rho^{2}-37 \rho+3\right) .
$$

De (2.18) e (2.17) segue

$$
s \rho>\frac{1}{50}+\frac{1}{25}\left(32 \rho^{2}-37 \rho+3\right)(1-s) .
$$

Ao substituir $s=\frac{1 / 2-4 \rho}{1-4 \rho}$ e $1-s=\frac{1 / 2}{1-4 \rho}$ em $(2.19)$ e multiplicar pelo real positivo $1-4 \rho$ obtemos

$$
33 \rho>116 \rho^{2}+2
$$

o qual é uma contradição para todo $\rho \leq \frac{33-\sqrt{161}}{232}=c$, isto implica que G não é $(1 / 2,1 / 50)$-denso como foi suposto, o que finaliza a prova da Proposição 1.4. 


\section{Capítulo 3}

\section{Grafos densos livres de triângulo}

Como observado no Capítulo 1, os resultados de Zykov [Zyk49], Tutte [Des48] (cf. [Erd62]) e Mycielski [Myc55] garantem que, para todo $k$ existe um grafo $\mathrm{G}$ livre de triângulo com número cromático $\chi(\mathrm{G})>k$. Observe que, fixado um grafo livre de triângulo $\mathrm{G}$ com $\chi(\mathrm{G})>k$, para cada $\varepsilon>0$, podemos escolher um grafo bipartido completo $\mathrm{G}^{\prime}$ de ordem suficientemente grande tal que a união disjunta de $\mathrm{G}$ e $\mathrm{G}^{\prime}$ produz um grafo de ordem $n$ com $\left(\frac{1}{4}-\varepsilon\right) n^{2}$ arestas, número cromático maior que $k$ e grau mínimo $o(n)$. Erdős e Simonovits [ES73] perguntaram quão grande pode ser o grau mínimo de um grafo livre de triângulo de número cromático $k$ ? Eles acreditavam que em grafos Hlivres, grau mínimo grande força número cromático acotado. Eles conjecturaram em particular que para grafos livres de triângulo, $\delta(\mathrm{G})>n / 3$ deveria implicar $\chi(\mathrm{G}) \leq 3$. Häggkvist [Häg82] achou um contraexemplo a esta conjectura. O grafo da Figura 7 corresponde-se a um blow-up não balanceado do grafo de Grötzsch, um grafo 4-cromático livre de triângulo de ordem $n(=29)$ com $\delta(\mathrm{G})>\frac{n}{3}$. No entanto, Thomassen [Tho02] mostrou que para todo $\varepsilon>0$, se $\delta(G)>\left(\frac{1}{3}+\varepsilon\right) n$ então $\chi(G)<c$ para alguma constante $c=c(\varepsilon)$. Finalmente, Brandt e Thomassé [BT10] mostraram que, de fato, $\delta(\mathrm{G})>n / 3$ implica $\chi(\mathrm{G}) \leq 4$.

Goddard e Lyle [GL11] e Nikiforov [Nik10] estenderam este resultado para grafos $\mathrm{K}_{r}$-livres, para todo $r \geq 3$. Em [GL11] e [Nik10] prova-se que $\delta(\mathrm{G})>\frac{2 r-5}{2 r-3} n$ implica $\chi(\mathrm{G}) \leq r+1$.

Allen, Böttcher, Griffiths, Kohayakawa e Morris $\left[\mathrm{ABG}^{+} 13\right]$ provaram um resultado análogo ao resultado de Thomassen quando proibimos qualquer grafo $\mathrm{H}, \mathrm{com} \chi(\mathrm{H}) \geq 3$, i.e. eles determinaram o limiar cromático $\delta_{\chi}(\mathrm{H})$ para todo grafo H. O limiar cromático $\delta_{\chi}(\mathrm{H})$ tem a propriedade que para $d<\delta_{\chi}(\mathrm{H})$, o número cromático de grafos $\mathrm{G}$ com $n$ vértices H-livres e mínimo grau $d n$, pode ser arbitrariamente grande, entanto que para $d>\delta_{\chi}(\mathrm{H}), \chi(\mathrm{G})$ é acotado.

Hajnal (cf. i.b. [ES73]) mostrou exemplos de grafos livres de triângulo de ordem $n$ com grau mínimo maior que $(1 / 3-\varepsilon) n$ e número cromático arbitrariamente grande. A construção é como segue: Fixe inteiros $k \ll m \ll l$, onde $2 m+k$ divide $l$. Inicie com a união disjunta do grafo bipartido completo $K_{l, 2 l}$ e o grafo de Kneser $\mathrm{GKne}_{2 m+k, k}$. Divida a partição de tamanho $2 l$ do grafo $\mathrm{K}_{l, 2 l} \mathrm{em}$ $2 m+k$ classes de igual tamanho e enumere estas classes como $X_{1}, \ldots, X_{2 m+k}$. Para cada vértice $v$ no grafo de Kneser, adicione todas as arestas de $v$ a todos os vértices da classe $X_{i}$ sempre que $i \in U_{v}$, onde $U_{v}$ é o $k$-subconjunto de $\left\{\begin{array}{c}{[2 m+k]} \\ k\end{array}\right\}$ que representa $v$ no grafo de Kneser (observe que isto evita triângulos). Pelo célebre resultado de Lovász (1976), $\chi\left(\mathrm{GKne}_{n, k}\right)=n-2 k+2$, assim, este grafo tem número cromático $k+2$, é livre de triângulos e no é difícil ver que seu grau mínimo pode fazer-se tão perto a $n / 3$ como se quiser.

Jin [Jin93] mostrou que, se G é um grafo de ordem $n$ livre de triângulo com $\delta(G)>\frac{i+1}{3 i+2} n$, para algum $1 \leq i \leq 9$, então $\mathrm{G} \rightarrow \Gamma_{i}$, i.e. $\mathrm{G}$ é homomorfo ao grafo de Andrásfai $\Gamma_{i}$ (cf. Definição 2.2). Chen, Jin e Koh [CJK97] provaram que um grafo G de ordem $n$ livre de triângulo com $\delta(G)>n / 3$ é homomorfo a algum grafo de Andrásfai $\Gamma_{i}$, e portanto $\chi(\mathrm{G}) \leq 3$, ou contém o grafo de Grötzsch GGrö como subgrafo, e portanto $\chi(G) \geq 4$ (veja Brandt [Bra99] para uma prova mais simples deste resultado). Segue que se $\mathrm{G}$ é livre de triângulo com $\delta(\mathrm{G})>n / 3$ e $\chi(\mathrm{G}) \leq 3$ então $\mathrm{G} \rightarrow \Gamma_{i}$. Por outro 
lado, o resultado em [BMRS17] garante que, se $\mathrm{G} \rightarrow \Gamma_{i}$ então $e_{1 / 2}(\mathrm{G}) \leq n^{2} / 50$.

Para grafos livres de triângulo com $\delta(G)>n / 3$ e $\chi(\mathrm{G})=4$ nós mostramos que $\boldsymbol{e}_{1 / 2}(\mathrm{G})<n^{2} / 54$ se G é regular. Para comodidade do leitor, apresentamos novamente o enunciado do Teorema 1.5.

Teorema 1.5. Seja G um grafo regular de ordem $n$ livre de triângulo. Se o grau de G excede $n / 3$ então $e_{1 / 2}(\mathrm{G}) \leq n^{2} / 50$. Se além disso, G não é 3 -cromático, então $e_{1 / 2}(\mathrm{G})<n^{2} / 54$.

O Teorema 1.5 é provado na Seção 3.1. Sua prova está fortemente baseada nas propriedades estruturais dos, assim chamados, grafos Vega descritos por Brandt e Thomassé em [BT10]. Isto melhora um resultado em Krivelevich [Kri95, Theorem 3] quem verificou a Conjectura 1.2 se G é $d$-regular com grau $d \geq 2 n / 5$.

Lembramos que um homomorfismo de grafos $\mathbf{G} \rightarrow \mathrm{H}$ é um mapeamento $f: V(\mathrm{G}) \rightarrow V(\mathrm{H})$ tal que se $u v \in E(\mathrm{G})$ então $f(u) f(v) \in E(\mathrm{H})$. Se existir $\mathrm{G} \rightarrow \mathrm{H}$ nós dizemos que G é homomorfo a $\mathrm{H}$ ou que G é H-tipo. Um automorfismo é um homomorfismo sobrejetivo de um grafo em se mesmo. Um homomorfismo bijetivo é um isomorfismo. Um subgrafo $\mathrm{H}$ de $\mathrm{G}$ é um núcleo de $\mathrm{G}$ se existir $\mathrm{G} \rightarrow \mathrm{H}$ mas não $\mathrm{G} \rightarrow \mathrm{H}^{\prime}$ para qualquer subgrafo próprio $\mathrm{H}^{\prime}$ de $\mathrm{H}$. Assim, $\mathrm{H}$ é o mais pequeno subgrafo (induzido) de G que é sua imagem homomorfa. A família de subgrafos H de G para os quais $G \rightarrow H$ é não vazia. Um elemento minimal (com respeito à relação de ordem parcial "é subgrafo de") nesta família é um núcleo. Portanto, todo grafo tem um núcleo. Este núcleo é único a menos de isomorfismos: se $\mathrm{H}_{1}$ e $\mathrm{H}_{2}$ são núcleos de $\mathrm{G}$ com mapeamentos $f_{1}: V(\mathrm{G}) \rightarrow V\left(\mathrm{H}_{1}\right)$ e $f_{2}: V(\mathrm{G}) \rightarrow V\left(\mathrm{H}_{2}\right)$ respectivamente, então as restrições de $f_{1}$ a $\mathrm{H}_{2}$ e de $f_{2}$ a $\mathrm{H}_{1}$ são mapeamentos sobrejetivos e portanto $\mathrm{H}_{1}$ e $\mathrm{H}_{2}$ são isomorfos. Um grafo G que é seu próprio núcleo é chamado simplesmente de núcleo. Neste caso, todo homomorfismo $\mathrm{G} \rightarrow \mathrm{G}$ é sobrejetivo, i.e. um automorfismo. Um grafo bipartido (não vazio) é seu próprio núcleo se e só se é isomorfo a $\mathrm{K}_{2}$. Decidir se um grafo é seu próprio núcleo é um problema NP-completo (ainda entre grafos 3-coloríveis [HN92]).

Dois vértices são similares se as suas vizinhanças coincidem. Para um vértice $v \in V(\mathrm{G})$, nós denotamos $\mathcal{S}(v) \stackrel{\text { def }}{=}\{u \in V: N(u)=N(v)\}$ a classe de similaridade de $v$ e $\mathcal{S}(v) \stackrel{\text { def }}{=}|\mathcal{S}(v)|$ o tamanho desta classe. Observe que o núcleo de um grafo G pode ser obtido por eliminação sucessiva de vértices similares.

Brandt [Bra99] mostrou que todo grafo livre de triângulo com $\delta(\mathrm{G}) \geq|\mathrm{G}| / 3$ não homomorfo a $\Gamma_{i}$ contém $\mathrm{GPet} / e$ como subgrafo. $\mathrm{GPet} / e$ é o grafo de 9 vértices que resulta de contrair uma aresta ao grafo de Petersen.

Teorema 3.1 (Brandt [Bra99]). Seja G um grafo livre de triângulo de ordem $n$ com grau mínimo $\delta(\mathrm{G}) \geq n / 3$. Então $\mathrm{G}$ não é homomorfo a $\Gamma_{i}$ se e só se $\mathrm{G}$ contém $\mathrm{GPet/e} \mathrm{como} \mathrm{subgrafo.}$

$\mathrm{O}$ grafo $\mathrm{aPet} / e$ é um grafo núcleo e contém um $\mathrm{C}_{6}$ induzido. O seguinte lema é um corolário ao Teorema 3.1.

Lema 3.2 (Brandt [Bra99]). Se G é um grafo livre de triângulo de grau mínimo $\delta(\mathrm{G})>n / 3$ não homomorfo a um grafo de Andrásfai, então $\mathrm{G}$ contém um $\mathrm{C}_{6}$ induzido e um vértice dominando três vértices deste $\mathrm{C}_{6}$.

A existência de um vértice que domina três vértices neste $\mathrm{C}_{6}$ é garantida porque, caso contrário, a relação

$$
6 \delta(\mathrm{G}) \leq \sum_{v \in V\left(\mathrm{C}_{6}\right)} d(v) \leq 2 n
$$

é verificada, contradizendo a condição do grau mínimo para G.

Chen, Jin e Koh [CJK97] provaram que se $\delta(\mathrm{G})>n / 3$ então G é homomorfo a um grafo de Andrásfai $\Gamma_{i}$ (e portanto é 3-colorível) ou contém o grafo de Grötzsch como subgrafo (e portanto $\chi(\mathrm{G}) \geq 4)$. 


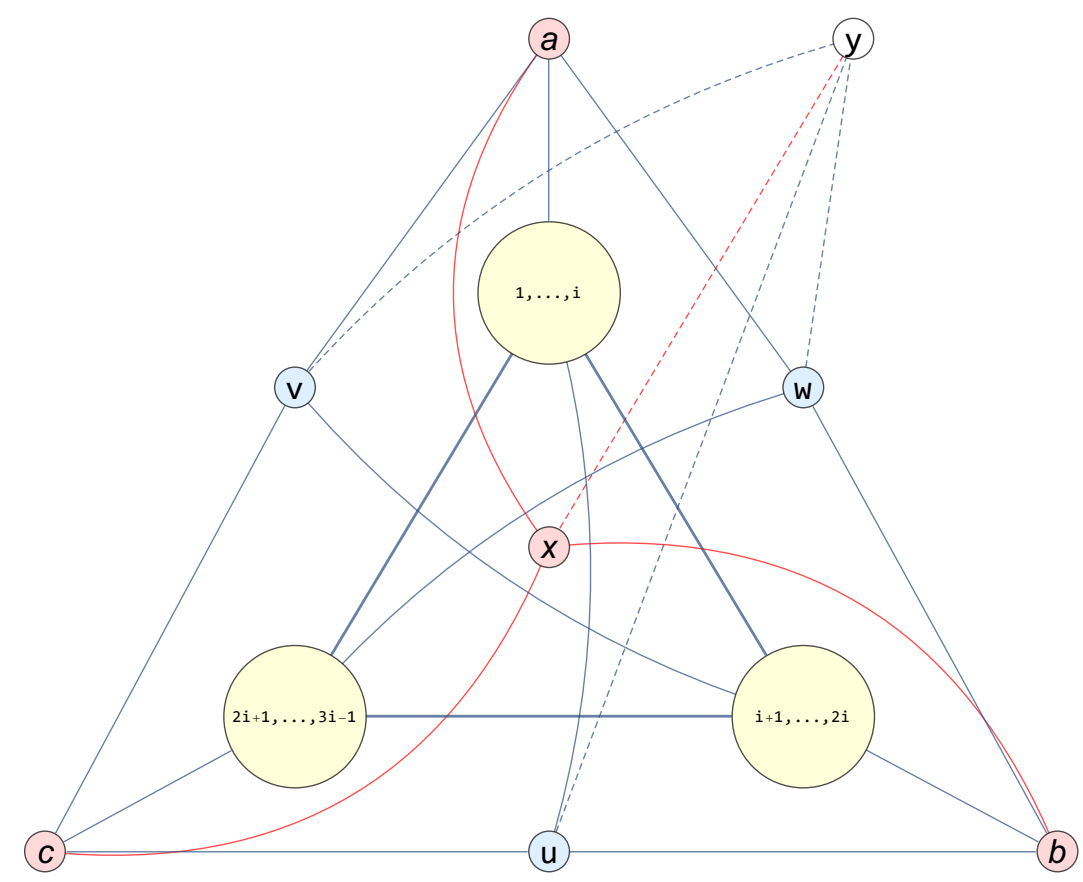

Figura 5: Estrutura global de um grafo Vega

Teorema 3.3 (Chen, Jin e Koh [CJK97]). Seja G um grafo livre de triângulo de ordem $n$ com grau mínimo $\delta(\mathrm{G})>n / 3$. Então $\mathrm{G}$ não é homomorfo com algum grafo de Andrásfai $\Gamma_{i}$ se e só se $\mathrm{G}$ contém o grafo de Grötzsch como subgrafo.

Finalmente, Brandt e Thomassé [BT10] provam que $\delta(\mathrm{G})>n / 3$ implica $\mathrm{G} \rightarrow \Upsilon_{i}$. O grafo $\Upsilon_{i}$ é um membro de uma família infinita de grafos núcleos 4-cromáticos conhecidos como grafos Vega e introduzidos por Brandt e Pisanski [BP98] com ajuda do programa de computador VEGA [Pis95]. ${ }^{1}$

Definição 3.4 (Grafos Vega). Para cada $i \geq 2$, definimos os quatro grafos Vega $\Upsilon_{i}, \Upsilon_{i}-\{y\}, \Upsilon_{i}-\{2 i\}$ e $\Upsilon_{i}-\{y, 2 i\}$ como segue. Inicie com a união disjunta do grafo de Andrásfai $\Gamma_{i}$, uma aresta $x y$ e o 6-ciclo avcubw. Adicione arestas unindo $x$ a cada vértice em $\{a, b, c\}$ e $y$ a cada vértice em $\{u, v, w\}$. Adicione as $2 i$ arestas possíveis desde o conjunto $\{a, u\}$ ao conjunto $\{1, \ldots, i\} \subset V\left(\Gamma_{i}\right)$; as $2 i$ arestas desde $\{b, v\}$ a $\{i+1, \ldots, 2 i\} \subset V\left(\Gamma_{i}\right)$; e finalmente as $2(i-1)$ arestas desde $\{c, w\}$ a $\{2 i+1, \ldots, 3 i-1\} \subset V\left(\Gamma_{i}\right)$. O grafo formado é o grafo Vega $\Upsilon_{i}$ de $3 i+7$ vértices. Os outros três grafos Vega são formados a partir de $\Upsilon_{i}$ por eliminação de vértices: os dois grafos Vega de $3 i+6$ vértices $\Upsilon_{i}-\{y\}$ e $\Upsilon_{i}-\{2 i\}$, e o grafo Vega de $3 i+5$ vértices $\Upsilon_{i}-\{y, 2 i\}$.

A Figura 5 mostra a estrutura geral de um grafo Vega.

É rotina verificar que os grafos Vega são núcleos (cf. [BT10]). Da Definição 3.4 segue que para todo $i \geq 2$ temos $\Upsilon_{i}-\{y, 2 i\} \rightarrow \Upsilon_{i}-\{y\} \rightarrow \Upsilon_{i}$ e $\Upsilon_{i}-\{y, 2 i\} \rightarrow \Upsilon_{i}-\{2 i\} \rightarrow \Upsilon_{i}$. Além disso, para todo $j>i$ temos $\Upsilon_{i}-\{y, 2 i\} \rightarrow \Upsilon_{j}-\{y, 2 j\}, \Upsilon_{i}-\{y\} \rightarrow \Upsilon_{j}-\{y\}, \Upsilon_{i}-\{2 i\} \rightarrow \Upsilon_{j}-\{2 j\}$ e $\Upsilon_{i} \rightarrow \Upsilon_{j}$. Em particular, $\Upsilon_{2}-\{y, 4\}$ (o grafo de Grötzsch) é homomorfo a todos os outros grafos Vega. Também temos que $\Gamma_{i} \rightarrow \Upsilon_{i}$, onde $\Gamma_{i}$ é o $i$-ésimo grafo de Andrásfai.

Teorema 3.5 (Brandt e Thomassé [BT10]). Seja G um grafo livre de triângulo de ordem $n$ e grau mínimo $\delta(\mathrm{G})>n / 3$. Então, $\mathrm{G} \rightarrow \Upsilon_{i}$ para algum inteiro $i \geq 2$. Além disso, se $\mathrm{G}$ não é 3colorível, então existe um homomorfismo sobrejetivo $f: V(\mathrm{G}) \rightarrow V(\mathrm{H})$, onde $\mathrm{H}$ é um dos grafos Vega $\Upsilon_{i}-\{y, 2 i\}, \Upsilon_{i}-\{y\}, \Upsilon_{i}-\{2 i\}$ ou $\Upsilon_{i}$, para algum inteiro $i \geq 2$.

\footnotetext{
${ }^{1}$ Pisanski é um dos desenvolvedores principais de VEGA, cf. [FG05, p. x]
} 

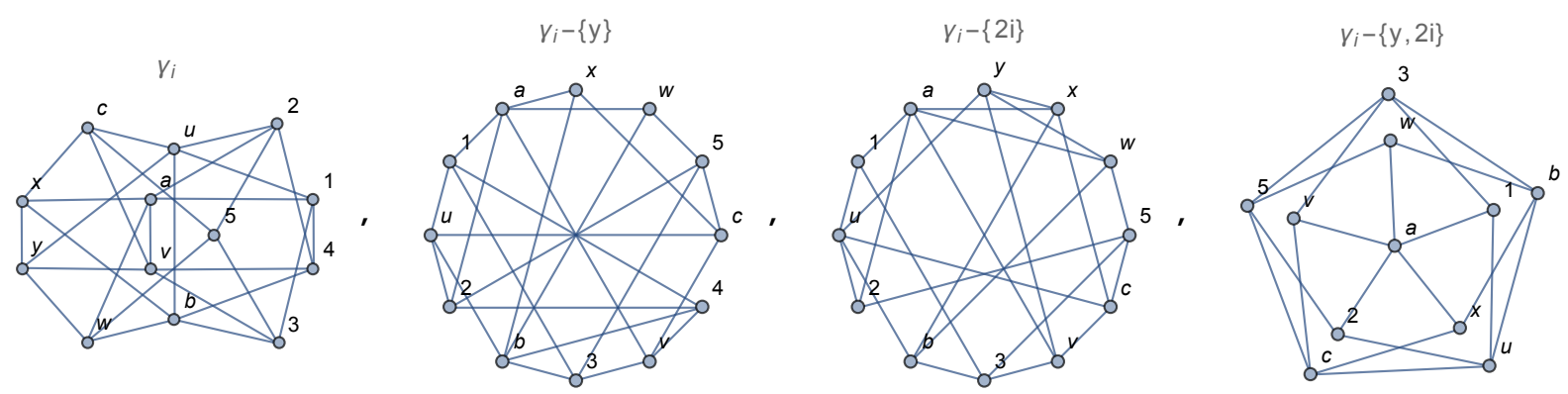

Figura 6: Grafos Vega para $i=2$

Corolário 3.6. Seja G um grafo livre de triângulo de ordem n e grau mínimo $\delta(G)>n / 3$. Então, G é 4-colorível.

Um grafo ponderado é um par $(\mathrm{H}, w)$ onde H é um grafo e $w: V(\mathrm{H}) \rightarrow[0,1]$ é a função peso que satisfaz

$$
w(V(\mathrm{H})) \stackrel{\text { def }}{=} \sum_{v \in V(\mathrm{H})} w(v)=1
$$

$\mathrm{O}$ conjunto de vértices do grafo ponderado $(\mathrm{H}, w)$ é o conjunto $V(\mathrm{H})$. O grau de cada vértice $v$ num grafo ponderado é $\sum_{u \in N_{\mathrm{H}}(v)} w(u)$ e o mínimo grau é denotado $\delta((\mathrm{H}, w))$. O peso de um subconjunto de vértices $S \subset V(\mathrm{H})$ é $w(S) \stackrel{\text { def }}{=} \sum_{v \in S} w(v)$. Se $w\left(N_{\mathrm{H}}(v)\right)$ é constante para todo vértice $v$ de $(\mathrm{H}, w)$ então dizemos que $w$ é regular. A função de peso é positiva se $w(v)>0$ para todo vértice de $\mathrm{H}$. Nós definimos o número de arestas do grafo ponderado $(\mathrm{H}, w)$ como

$$
\mathfrak{e}((\mathrm{H}, w)) \stackrel{\text { def }}{=} \frac{1}{2} \sum_{v \in V(\mathrm{H})} w(v) w\left(N_{\mathrm{H}}(v)\right) .
$$

Se a função de peso $w$ toma valores racionais então $(\mathrm{H}, w)$ corresponde-se ao grafo simples G que resulta de fazer o correspondente blow-up não balanceado de H, i.e., de substituir cada vértice $v_{i} \in V(\mathrm{H})$ por um conjunto independente $V_{i}$ de tamanho $\left|V_{i}\right|=w\left(v_{i}\right) n$ onde $n$ é o mínimo comum múltiplo dos denominadores nos pesos $w\left(v_{i}\right), v_{i} \in V(\mathrm{G})$; e cada aresta $v_{i} v_{j} \in E(\mathrm{H})$ pelo grafo bipartido completo com partições $V_{i}$ e $V_{j}$. Reciprocamente, todo grafo simples G de ordem $n$ corresponde-se com o grafo ponderado $(\mathrm{H}, w)$ onde $\mathrm{H}$ é o núcleo de $\mathrm{G}$ e o peso de cada vértice $v \in V(\mathrm{H})$ é $w(v)=s(v) / n$, onde $s(v)$ é o tamanho em G da classe de similaridade de $v$.

Resolvendo um problema de programação inteira, é possível notar que cada grafo Vega admite uma função de peso regular, positiva e de valores racionais com grau mínimo maior que $1 / 3$. Esta função de peso é única (cf. Brandt e Thomassé [BT10, Theorem 3]).

Lema 3.7 ([BT10, Theorem 3]). Todo grafo Vega $\mathrm{H}$ admite uma única função de peso regular positiva w tal que o grau $\delta$ do grafo ponderado $(\mathrm{H}, w)$ excede $1 / 3$.

Prova. Para cada $i \geq 2$ defina $w$ como segue:

No grafo $\Upsilon_{i}$, atribuir os seguintes pesos: $\frac{1}{27 i-19}$ nos vértices $x, y, 1$ e $2 i ; \frac{3 i-3}{27 i-19}$ a $c$ e $w ; \frac{3 i-2}{27 i-19}$ a $a, b, u$ e $v$; e peso $\frac{3}{27 i-19}$ para os outros vértices.

Em $\Upsilon_{i}-\{y\}$, atribuir os seguintes pesos: $\frac{1}{27 i-22}$ a 1 e $2 i ; \frac{2}{27 i-22}$ a $x ; \frac{3 i-4}{27 i-22}$ a $w ; \frac{3 i-3}{27 i-22}$ a $u, v, c$; $\frac{3 i-2}{27 i-22}$ a $a, b$, e $\frac{3}{27 i-22}$ a os outros vértices.

Em $\Upsilon_{i}-\{2 i\}$, peso $\frac{1}{27 i-22}$ nos vértices $x$ e $y ; \frac{2}{27 i-22}$ a 1 e $i ; \frac{3 i-3}{27 i-22}$ a $b, c, v$ e $w ; \frac{3 i-2}{27 i-22}$ a $a$ e $u$; e $\frac{3}{27 i-22}$ a os outros vértices. 


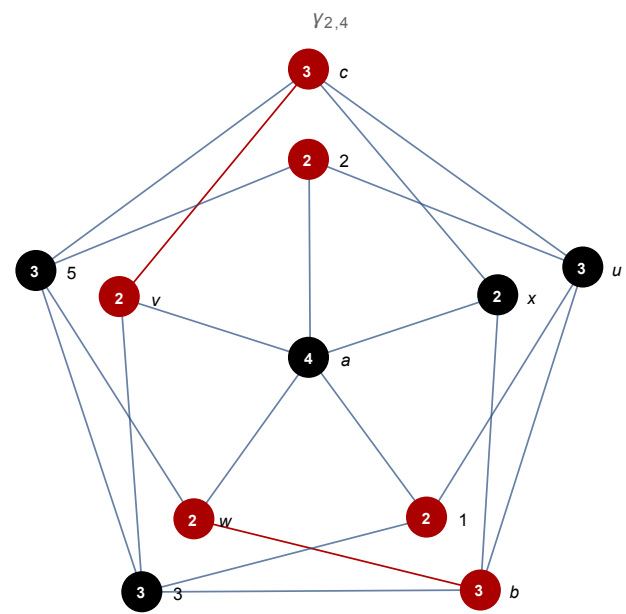

Figura 7: Blow-up do grafo de Grötzsch de 29 vértices

Em $\Upsilon_{i}-\{y, 2 i\}$, peso $\frac{1}{27 i-25}$ a 1 e $2 i ; \frac{2}{27 i-25}$ a $x ; \frac{3 i-4}{27 i-25}$ a $w ; \frac{3 i-3}{27 i-25}$ a $c, u$ e $v ; \frac{3 i-2}{27 i-25}$ a $a$ e $b$; e peso $\frac{3}{27 i-25}$ a os outros vértices.

Denotando $X=N(a) \cap N(u), Y=N(b) \cap N(v)$, e $Z=N(c) \cap N(w)$, a Tabela 3 resume a função de peso regular do Lema 3.7.

\begin{tabular}{c|c|c|c|c|c|c|c|c|c|c|c|c|c|c|c|c}
\cline { 2 - 11 } & $x$ & $y$ & $a$ & $b$ & $c$ & $u$ & $v$ & $w$ & $X \backslash\{1, i\}$ & 1 & $i$ & $Y \backslash\{2 i\}$ & $2 i$ & $Z$ & $\delta$ & $w(V)$ \\
\hline$\Upsilon_{i}$ & 1 & 1 & $3 i-2$ & $3 i-2$ & $3 i-3$ & $3 i-2$ & $3 i-2$ & $3 i-3$ & $3 i-6$ & 1 & 3 & $3 i-2$ & 1 & $3 i-3$ & $9 i-6$ & $27 i-19$ \\
\hline$\Upsilon_{i}-\{y\}$ & 2 & - & $3 i-2$ & $3 i-2$ & $3 i-3$ & $3 i-3$ & $3 i-3$ & $3 i-4$ & $3 i-6$ & 1 & 3 & $3 i-2$ & 1 & $3 i-3$ & $9 i-7$ & $27 i-22$ \\
\hline$\Upsilon_{i}-\{2 i\}$ & 1 & 1 & $3 i-2$ & $3 i-3$ & $3 i-3$ & $3 i-2$ & $3 i-3$ & $3 i-3$ & $3 i-6$ & 2 & 2 & $3 i-3$ & - & $3 i-3$ & $9 i-7$ & $27 i-22$ \\
\hline$\Upsilon_{i}-\{y, 2 i\}$ & 2 & - & $3 i-2$ & $3 i-3$ & $3 i-3$ & $3 i-3$ & $3 i-4$ & $3 i-4$ & $3 i-6$ & 2 & 2 & $3 i-3$ & - & $3 i-3$ & $9 i-8$ & $27 i-25$ \\
\hline
\end{tabular}

Tabela 3.1: Função de peso regular para grafos Vega. Cada celda contém o numerador do peso racional do respectivo vértice. Nas columnas indexadas por conjuntos, a celda contém a soma (dos numeradores) dos pesos dos vértices no conjunto

\subsection{Prova do Teorema 1.5}

Teorema 1.5. Seja G um grafo regular de ordem $n$ livre de triângulo. Se o grau de G excede $n / 3$ então $e_{1 / 2}(\mathrm{G}) \leq n^{2} / 50$. Se além disso, G não é 3 -cromático, então $e_{1 / 2}(\mathrm{G})<n^{2} / 54$.

Prova. Seja G um grafo $d$-regular de ordem $n$ livre de triângulo, com $d>n / 3$. Pelo Corolário 3.6 temos $\chi(G) \leq 4$. Se G é bipartido então $e_{1 / 2}(G)=0$. Se $\chi(G)=3$ então pelo Teorema 3.3 temos que $\mathrm{G} \rightarrow \Gamma_{j}$ para algum grafo de Andrásfai $\Gamma_{j}$, e pelo Teorema 2.7 temos $e_{1 / 2}(\mathrm{G}) \leq n^{2} / 50$. Assim, podemos assumir que $\chi(\mathrm{G})=4$. Substituindo cada classe de vértices similares $\mathcal{S}(v)$ em $\mathrm{G}$ por um vértice com peso $s(v) / n$, nós construímos um grafo ponderado (H, $w$ ). O Teorema 3.5 garante que H é um grafo Vega. Pela construção de $(\mathrm{H}, w)$, temos que $w$ é uma função peso positiva, e desde que G é regular, $w$ é regular. O Lema 3.7 garante a unicidade de $w$. Dividimos a prova por casos dependendo de qual grafo Vega é H.

Caso 1. $\mathrm{H}=\Upsilon_{i}-\{y, 2 i\}$. Para o caso particular $i=2$ (lembramos que o grafo $\Upsilon_{2}-\{y, 4\}$ é isomorfo a GGrö), a Figura 7 mostra o grafo 10-regular de ordem 29 obtido de um blow-up não balanceado do grafo GGrö. Neste grafo, um subconjunto de $\lfloor 29 / 2\rfloor=14$ vértices induz $12<29^{2} / 54$ arestas.

Considere $i \geq 3$. Em $\Upsilon_{i}-\{y, 2 i\}$ o 6-ciclo $(a, v, c, u, b, w)$ é induzido e $x$ domina $\{a, b, c\}$. 
Considere o conjunto

$$
X=\left(N_{\mathrm{H}}(a) \backslash\{x\}\right) \cup\{b, c\},
$$

Da definição de $w$ para $\Upsilon_{i}-\{y, 2 i\}$ no Lema 3.7 segue que, para cada $i \geq 3$,

$$
\begin{aligned}
w(X) & =\sum_{v \in X} w(v)=\frac{15 i-16}{27 i-25}>\frac{1}{2}, \mathrm{e} \\
w(X \backslash\{b\}) & =\frac{12 i-13}{27 i-25}<\frac{1}{2} .
\end{aligned}
$$

Para provar o resultado basta mostrar que existe uma função peso $w^{\prime}$ com peso total $w^{\prime}(V(\mathrm{H}))=\frac{1}{2}$, tal que $e\left(\left(\mathrm{H}, w^{\prime}\right)\right)<\frac{1}{54}$. Definimos $\boldsymbol{w}^{\prime}$ como segue

$$
w^{\prime}(z)= \begin{cases}0 & z \in V(\mathrm{H}) \backslash X \\ w(z) & z \in X \backslash\{b\} \\ \frac{1}{2}-w(X \backslash\{b\}) & z=b\end{cases}
$$

Portanto, da definição de $\Upsilon_{i}-\{y, 2 i\}$ obtemos

$$
e\left(\left(\mathrm{H}, w^{\prime}\right)\right)=v c+w\left(\frac{1}{2}-v-w-c-\Phi\right),
$$

onde $\Phi=w(N(a) \backslash\{x, v, w\})=w(\{1, \ldots, i\})=\frac{3 i-2}{27 i-25}$ e por simplicidade temos denotado, para cada vértice $z \in V(\mathrm{H})$, o peso $w(z)$ simplesmente como $z$. Por um simples cálculo, do Lema 3.7 obtemos

$$
e\left(\left(\mathrm{H}, w^{\prime}\right)\right)=v c+w\left(\frac{1}{2}-v-w-c-\Phi\right)=\frac{(3 i-4)(9 i-5)}{2(27 i-25)^{2}}<\frac{1}{54}
$$

para todo $i \geq 3$.

Caso $2 . \mathrm{H}=\Upsilon_{i}-\{2 i\}$. Similarmente, no grafo $\Upsilon_{i}-\{2 i\}$ considere o conjunto

$$
X=\left(N_{\mathrm{H}}(a) \backslash\{x\}\right) \cup\{b, c\} .
$$

Da definição de $w$ dada para $\Upsilon_{i}-\{2 i\}$ pelo Lema 3.7 , nós obtemos para todo $i \geq 2$

$$
\begin{aligned}
w(X) & =\frac{15 i-14}{27 i-22}>\frac{1}{2}, \\
w(X \backslash\{b\}) & =\frac{12 i-11}{27 i-22}<\frac{1}{2} .
\end{aligned}
$$

Definimos a função de peso $w^{\prime}$ como

$$
w^{\prime}(z)= \begin{cases}0 & z \in V(\mathrm{H}) \backslash X \\ w(z) & z \in X \backslash\{b\} \\ \frac{1}{2}-w(X \backslash\{b\}) & z=b\end{cases}
$$

Portanto, $w^{\prime}(V(\mathrm{H}))=1 / 2$ e da definição de $\Upsilon_{i}-\{2 i\}$, temos

$$
e\left(\left(\mathrm{H}, w^{\prime}\right)\right)=v c+w\left(\frac{1}{2}-v-w-c-\Phi\right),
$$

onde $\Phi=w(N(a) \backslash\{x, v, w\})=\frac{3 i-2}{27 i-22}$ e por simplicidade temos denotado novamente o peso 
$w(z)$ simplesmente como $z$. Do Lema 3.7 obtemos

$$
e\left(\left(\mathrm{H}, w^{\prime}\right)\right)=v c+w\left(\frac{1}{2}-v-w-c-\Phi\right)=\frac{9(i-1)(3 i-2)}{2(27 i-22)^{2}}<\frac{1}{54}
$$

para todo $i \geq 2$.

Caso $3 . \mathrm{H}=\Upsilon_{i}-\{y\}$. Similarmente aos casos anteriores, mas nós excluímos de $X$ o vértice $w$ em vez de $b$, i.e. $\operatorname{com} X=\left(N_{\mathrm{H}}(a) \backslash\{x\}\right) \cup\{b, c\}$, do Lema 3.7 obtemos para todo $i \geq 2$

$$
\begin{aligned}
w(X) & =\frac{15 i-14}{22-27 i}>\frac{1}{2}, \\
w(X \backslash\{w\}) & =\frac{2(6 i-5)}{27 i-22}<\frac{1}{2} .
\end{aligned}
$$

Definimos a função de peso $w^{\prime}$ como

$$
w^{\prime}(z)= \begin{cases}0 & z \in V(\mathrm{H}) \backslash X \\ w(z) & z \in X \backslash\{w\}, \\ \frac{1}{2}-w(X \backslash\{w\}) & z=w\end{cases}
$$

assim, $w^{\prime}(V(\mathrm{H}))=1 / 2$ e da definição de $\Upsilon_{i}-\{y\}$ segue

$$
e\left(\left(\mathrm{H}, w^{\prime}\right)\right)=v c+b\left(\frac{1}{2}-v-b-c-\Phi\right)
$$

onde $\Phi=w(N(a) \backslash\{x, v, w\})=\frac{3 i-2}{27 i-22}$ e temos denotado $w(z)$ como $z$ para todo $z \in V(\mathrm{H})$. Do Lema 3.7 obtemos

$$
e\left(\left(\mathrm{H}, w^{\prime}\right)\right)=v c+b\left(\frac{1}{2}-v-b-c-\Phi\right)=\frac{27 i^{2}-48 i+22}{2(27 i-22)^{2}}<\frac{1}{54}
$$

para todo $i \geq 2$.

Caso $4 . \mathrm{H}=\Upsilon_{i}$. Neste caso excluímos de $X$ o vértice $c$. Com $X=\left(N_{\mathrm{H}}(a) \backslash\{x\}\right) \cup\{b, c\}$, do Lema 3.7 obtemos, para todo $i \geq 2$,

$$
\begin{aligned}
w(X) & =\frac{3(5 i-4)}{27 i-19}>\frac{1}{2}, \\
w(X \backslash\{c\}) & =\frac{9-12 i}{19-27 i}<\frac{1}{2} .
\end{aligned}
$$

Definimos $w^{\prime}$ como

$$
w^{\prime}(z)= \begin{cases}0 & z \in V(\mathrm{H}) \backslash X \\ w(z) & z \in X \backslash\{c\} \\ \frac{1}{2}-w(X \backslash\{c\}) & z=c\end{cases}
$$

Assim, $w^{\prime}(V(\mathrm{H}))=1 / 2$. Da definição de $\Upsilon_{i}$ temos

$$
e\left(\left(\mathrm{H}, w^{\prime}\right)\right)=w b+v\left(\frac{1}{2}-v-w-b-\Phi\right)
$$

onde $\Phi=w(N(a) \backslash\{x, v, w\})=\frac{3 i-2}{27 i-19}$ e por simplicidade temos denotado o peso $w(z)$ simplesmente como $z$. Finalmente, do Lema 3.7 obtemos

$$
e\left(\left(\mathrm{H}, w^{\prime}\right)\right)=w b+v\left(\frac{1}{2}-v-w-b-\Phi\right)=\frac{(3 i-2)(9 i-7)}{2(27 i-19)^{2}}<\frac{1}{54}
$$


para todo $i \geq 2$. Isto completa a prova do Teorema 1.5.

Como corolário ao Teorema 1.5. nós verificamos a Conjectura 1.11 assintoticamente em grafos $d$-regulares de ordem $n$ livres de triângulo com $d>n / 3$.

Corolário 1.6. Seja G um grafo regular de ordem $n$ livre de triângulo. Se o grau de G excede $n / 3$ então $\mathrm{G}$ pode ser tornado bipartido pela remoção de no máximo $\left(\frac{1}{25}+o(1)\right) n^{2}$ arestas.

Prova. Seja $U \subset V(\mathrm{G})$ um subconjunto de vértices de G de tamanho $|U|=\lfloor n / 2\rfloor$ tal que $\mathfrak{e}(U)=e_{1 / 2}(\mathrm{G})$. Denote $U^{\mathrm{c}}=V(\mathrm{G}) \backslash U$. Contando em duas formas as arestas de cruzamento $E\left(U, U^{\mathrm{c}}\right)$, obtemos

$$
e\left(U, U^{\complement}\right)=\sum_{u \in U} d\left(u, U^{c}\right)=\sum_{u \in U}(d-d(u, U))=\left\lfloor\frac{n}{2}\right\rfloor d-2 e(U)
$$

e analogamente

$$
e\left(U, U^{\mathrm{c}}\right)=\sum_{u \in U^{\mathrm{c}}} d(u, U)=\left\lceil\frac{n}{2}\right\rceil d-2 e\left(U^{\mathrm{c}}\right) .
$$

De (3.5) e (3.6) segue que

$$
e\left(U^{\mathrm{c}}\right)-e(U)=\frac{d}{2}\left(\left\lceil\frac{n}{2}\right\rceil-\left\lfloor\frac{n}{2}\right\rfloor\right)=\left\{\begin{array}{lc}
0 & \text { se } n \text { é par } \\
\frac{d}{2} & \text { se } n \text { é ímpar }
\end{array} .\right.
$$

Ao eliminar as arestas em $\left.\mathrm{G}\right|_{U}$ e $\left.\mathrm{G}\right|_{U^{\mathrm{c}}}$ nós obtemos um grafo bipartido e temos eliminado no máximo

$$
e(U)+e\left(U^{\mathrm{c}}\right)=2 e(U)+\frac{d}{2} \mathbb{1}_{[n \text { é ímpar }]}
$$

arestas.

Pelo Corolário 3.6 temos $\chi(\mathrm{G}) \leq 4$. Se G é bipartido então claramente $e(U)=0$ e o resultado segue de (3.7) uma vez que $d / 2 \leq(1 / 25+o(1)) n^{2}$. Se $\chi(G)=3$ então o Teorema 2.7 garante que $e(U) \leq n^{2} / 50$ e de (3.7) concluímos que temos eliminado no máximo

$$
\left(\frac{1}{25}+\frac{d}{2 n^{2}}\right) n^{2}=\left(\frac{1}{25}+o(1)\right) n^{2}
$$

arestas. Analogamente, se $\chi(\mathrm{G})=4$, o Teorema 1.5 garante que $e(U) \leq n^{2} / 54$ e pela relação (3.7) temos eliminado no máximo

$$
\left(\frac{1}{27}+\frac{d}{2 n^{2}}\right) n^{2} \leq\left(\frac{1}{25}+o(1)\right) n^{2}
$$

arestas. 


\section{Capítulo 4}

\section{Uma conjectura de Erdős, Faudree, Rousseau e Schelp}

Um grafo fortemente regular (em diante $\operatorname{srg}$ ) com parâmetros $(n, d, \lambda, \mu)$, é um grafo $d$-regular de ordem $n$ tal que cada par de vértices adjacentes tem $\lambda$ vizinhos comuns e cada par de vértices no adjacentes tem $\mu$ vizinhos comuns. Claramente, srg livres de triângulo satisfazem $\lambda=0$. O principal resultado neste capítulo é mostrar um contraexemplo a uma conjectura de Erdős, Faudree, Rousseau e Schelp [EFRS94, Conjectura 1.1] para certos valores do parâmetro $\alpha$. O contraexemplo é dado pela família infinita de grafos $\mathrm{GCl} \mathrm{e}^{[16 n]}$, os blow-ups balanceados do grafo de Clebsch [Cle68], o único srg com parâmetros $(16,5,0,2)$.

Nós observamos que, em contraste com grafos $(3, t)$-Ramsey considerados em [EFRS94] para estabelecer a Conjectura 1.7 p. 4; blow-ups balanceados de srg livres de triângulo mostram densidade local maior para pequenos valores de $\alpha$. De fato, ao relaxar a nossa definição de srg a fim de permitir exemplos triviais (como grafos bipartidos completos de ordem par), a melhor cota inferior conhecida para a função $\beta\left(\alpha, \mathrm{K}_{3}\right)$ é fornecida por blow-ups balanceados de srg livres de triângulo para todo valor de $\alpha \in(0,1]$.

\subsection{Grafos de Ramsey}

Lembramos que uma $k$-coloração de um conjunto $S$ é uma função $c:[k] \rightarrow S$ que atribui $k$ cores a os elementos de $S$.

Definição 4.1. O número de Ramsey $R_{r}\left(s_{1}, s_{2}, \ldots, s_{k}\right)$ é o menor inteiro $n$ tal que toda $k$-coloração dos $r$-subconjuntos de $[n]$ contém um conjunto monocromático de tamanho $s_{i}$ para algum $1 \leq i \leq k$, onde um conjunto $S \subset[n]$ é chamado monocromático se todos os $r$-subconjuntos de $S$ são da mesma cor.

Se $r=2$ (grafos), nós omitimos o subíndice. Em 1929 Ramsey [Ram29] mostrou que estes números existem para todos os valores dos parâmetros. Determinar os números de Ramsey é um problema altamente não trivial e é um dos problemas fundamentais em matemáticas discretas (veja [GRS13] ou a recente monografia [XLL18] para uma exposição de problemas, novas conjecturas, e resultados parciais em teoria de grafos extremais, teoria de números, geometria discreta, analise funcional, desenho de algoritmos, entre outras áreas).

Os números de Ramsey da forma $R(3, t)$ tem recebido considerável atenção. De um resultado de Erdős e Szekeres [ES35] segue que $R(3, t) \leq\left(\begin{array}{c}t+1 \\ 2\end{array}\right)$. Graver e Yackel [GY68], Ajtai, Komlós e Szemerédi [AKS80] provaram $R(3, t) \leq c \cdot \frac{t^{2}}{\ln t}$ para alguma constante $c>0$. Griggs (1983) mostrou que $R(3, t) \leq \frac{5 t^{2}}{12 \ln t}$. Erdős [Erd61] mostrou que $R(3, t)=\Omega\left(t^{2} / \log ^{2} t\right)$. Finalmente, em 1995, Kim [Kim95] mostrou que $R(3, t)=\Theta\left(t^{2} / \log t\right)$ i.e. existem constantes $c_{1}>0$ e $c_{2}>0$ tais que

$$
c_{1} \cdot \frac{t^{2}}{\log t} \leq R(3, t) \leq c_{2} \cdot \frac{t^{2}}{\log t}
$$


O melhor valor conhecido para $c_{1}$ é 1/4 (em [BK13]). Em Shearer [She83, She91, She92, She95] implicitamente se mostra $c_{2}=1$.

Um grafo $\mathrm{G}$ de ordem $n$ e seu complemento $\mathrm{G}^{\mathrm{C}}$ podem ser considerados de forma natural como os subgrafos monocromáticos induzidos por uma 2-coloração apropriada das arestas de $\mathrm{K}_{n}$. Nós lembramos que um grafo G é $(s, t)$-Ramsey se $|\mathrm{G}|=R(s, t)-1$ e G não contém um grafo completo de $s$ vértices nem um conjunto independente de $t$ vértices. Assim, para pequenos valores de $t$, parece intuitivamente razoável que blow-ups de grafos $(3, t)$-Ramsey sejam grafos livres de triângulo com alta densidade local num intervalo apropriado de $\alpha$. Desde que $R(3,2)=3, R(3,3)=6, R(3,4)=9$, etc (cf. [Rad94]), segue que $\mathrm{K}_{2}$ é $(3,2)$-Ramsey, $\mathrm{C}_{5}$ é $(3,3)$-Ramsey e o grafo de Wagner aWag (isomorfo a GMöb 8 ) é $(3,4)$-Ramsey, etc.

\subsection{Um contraexemplo para a Conjectura 1.7}

Erdős, Faudree,Rousseau e Schelp [EFRS94, Theorem 2.4] provaram que para $n$ suficientemente grande, o grafo de Mantel $\mathrm{K}_{2}^{[2 n]}$ é extremal para o problema da densidade local em grafos livres de triângulo em todo o intervalo $[0.648,1]$, i.e. $\beta\left(\alpha, \mathrm{K}_{3}\right)=(2 \alpha-1) / 4$ para todo $0.648 \leq \alpha \leq 1$. Observe que este resultado generaliza o teorema de Mantel.

Krivelevich [Kri95] mostrou que $\mathrm{K}_{2}^{[2 n]}$ é ainda extremal para $\alpha \geq 0.6$.

Teorema 4.2 (Krivelevich [Kri95, Theorem 4]). Seja G um grafo de ordem n e $\alpha \geq 0.6$ fixo. Seja $\beta=(2 \alpha-1) / 4$. Se cada conjunto de an vértices de $\mathrm{G}$ induz mais que $\beta n^{2}$ arestas, então $\mathrm{G}$ contém um triângulo.

Nós observamos que blow-ups balanceados do grafo de Clebsch GCle fornecem um contraexemplo à Conjectura 1.7 .

\section{Teorema 1.8.}

$$
\beta\left(\alpha, \mathrm{K}_{3}\right) \geq \begin{cases}0, & 0<\alpha \leq \frac{5}{16} \\ \frac{1}{8}\left(\alpha-\frac{5}{16}\right), & \frac{5}{16}<\alpha \leq \frac{3}{8} \\ \frac{1}{64}(4 \alpha-1), & \frac{3}{8}<\alpha \leq \frac{1}{2} \\ \frac{1}{64}(16 \alpha-7), & \frac{1}{2}<\alpha \leq \frac{5}{8} \\ \frac{3}{128}(8 \alpha-3), & \frac{5}{8}<\alpha \leq \frac{11}{16} \\ \frac{5}{32}(2 \alpha-1), & \frac{11}{16}<\alpha \leq 1\end{cases}
$$

Como $\frac{4 \alpha-1}{64}>\frac{5 \alpha-2}{25}$ para todo $\frac{53}{120} \leq \alpha<\frac{103}{220}$, nós obtemos o seguinte corolário.

Corolário 1.9. A Conjectura 1.7 é falsa no intervalo $\alpha \in\left[\frac{53}{120}, \frac{103}{220}\right)$.

Observe que analogamente a como a equação (1.5) da Conjectura 1.7 foi obtida, nós podemos obter a equação (4.1) no Teorema 1.8 para a instância $k=1$, i.e para o grafo GCle. Nós podemos fazer isto sempre que o grafo seja computacionalmente tratável (de ordem pequeno), no entanto, para provar o Teorema 1.8 para todo inteiro $k$, precisa-se o seguinte lema, o qual garante que entre os conjuntos $U$ de tamanho $\lfloor\alpha n\rfloor$ que minimizam a densidade $e(U) / n^{2}$ num blow-up (não necessariamente balanceado) de um grafo $\mathrm{H}$, sempre podemos escolher um conjunto de vértices que contém no máximo uma classe do blow-up parcialmente. A prova do lema é simples e está implicitamente contida numa observação de Sudakov [KS03, p. 141]. Uma prova na terminologia de grafos fracionários é dada em Brandt [Bra98, Proposition 2.1]. Nós trasladamos o equivalente da prova em [Bra98] na terminologia de $\eta$-blow-ups.

Lema 4.3. Sejam $\mathrm{H}$ um grafo de ordem $h$ sobre o conjunto de vértices $[h]=\{1, \ldots, h\}$ e $\eta \in \mathbb{Z}_{+}^{h}$ um vetor de inteiros positivos. Seja $\mathrm{G}=H^{[\eta]}$ o $\eta$-blow-up de $\mathrm{H}$ e $V(\mathrm{G})=I_{1} \cup \ldots \cup I_{h}$ a partição de 
$V(\mathrm{G})$ em conjuntos independentes induzida por $\eta$. Sejam além $n=|\mathrm{G}|$ e $\alpha \in(0,1]$ um real. Se

$$
\beta=\min \left\{\frac{e(U)}{n^{2}}: U \subseteq V(G),|U|=\lfloor\alpha n\rfloor\right\},
$$

então existe um subconjunto $U \subseteq V(G)$, com $|U|=\lfloor\alpha n\rfloor$ e e $(U)=\beta n^{2}$, tal que $U$ contém ou é disjunto de cada $I_{k}$, para cada índice $k \in[h]$ exceto no máximo um.

Prova. Seja $U \subseteq V(G)$ um subconjunto de tamanho $|U|=\lfloor\alpha n\rfloor \operatorname{com} e(U)=\beta n^{2}$ que intersecta parcialmente a menor quantidade possível de classes $I_{k}$. Se $U$ intersecta parcialmente apenas uma classe então o lema verifica-se. Suponha pelo contrário que $U$ intersecta parcialmente pelo menos duas classes $I_{j}$ e $I_{k}$. Sem perda de generalidade, suponha que $\sum_{v \in I_{j}} d(v) \leq \sum_{v \in I_{k}} d(v)$. Seja $s=\min \left\{\left|I_{j} \backslash U\right|,\left|I_{k} \cap U\right|\right\}$ e $S_{1} \subseteq I_{j} \backslash U$ e $S_{2} \subseteq I_{k} \cap U$ de tamanhos $\left|S_{1}\right|=\left|S_{2}\right|=s$. Definimos $U^{\prime}=\left(U \backslash S_{2}\right) \cup S_{1}$. Claramente, $\left|U^{\prime}\right|=|U|$. Consideramos dois casos dependendo se $j k$ é uma aresta em H.

(i) Se $j k \notin E(\mathrm{H})$, então

$$
e\left(U^{\prime}\right)=e(U)+s\left(\sum_{v \in I_{j}} d(v)-\sum_{v \in I_{k}} d(v)\right) \leq e(U),
$$

(ii) Se $j k \in E(\mathrm{H})$, então

$$
e\left(U^{\prime}\right)=e(U)+s\left(\sum_{v \in I_{j}} d(v)-\sum_{v \in I_{k}} d(v)\right)-s^{2} \leq e(U) .
$$

Desde que $e(U)=\beta n^{2}$ é minimal e $\left|U^{\prime}\right|=\lfloor\alpha n\rfloor$, temos $e\left(U^{\prime}\right)=\beta n^{2}$. Mas agora, $U^{\prime}$ ou contém completamente $I_{j}$ ou é disjunta de $I_{k}$, em qualquer caso, $U^{\prime}$ intersecta parcialmente uma classe menos que $U$, isto contradiz a escolha $U$, o que completa a prova.

Desde que o número de classes em $\mathrm{GCl}^{[16 k]}$ permanece constante para todo $k$, o Lema 4.3 implica o Teorema 1.8 indutivamente dado que a densidade $e(U) / n^{2}$ também permanece constante. Para o caso base $k=1$ nós usamos um programa de computador que computa exaustivamente os $\left(\begin{array}{c}16 \\ \lfloor\alpha 16\rfloor\end{array}\right)$ subconjuntos de vértices de GCle de tamanho $\lfloor\alpha 16\rfloor$ para todo $\alpha \in(0,1]$ (cf. Anexo A). 


\section{Capítulo 5}

\section{Conclusões e considerações finais}

O problema de determinar o subgrafo bipartido máximo é NP-completo ainda em grafos de grau máximo 3 [Yan78]. O problema de partições judiciosas é NP-difícil [SS94] ainda no caso de bipartições. Krivelevich [Kri95] observou que para grafos regulares, o problema da densidade local é pelo menos tão difícil como o problema da remoção de arestas para tornar grafos bipartidos, i.e., se G é um grafo regular de $n$ vértices que contém um subconjunto $U$ de $\lfloor n / 2\rfloor$ vértices que expande no máximo $m$ arestas, então G pode ser tornado bipartido pela omissão de no máximo $2 m$ arestas. O Corolário 1.6 é equivalente a esta observação. Consequentemente, a Conjectura 1.2 é pelo menos tão difícil como a Conjectura 1.11 para grafos regulares. O grafo de Petersen mostra que a recíproca não vale, i.e. o grafo de Petersen não é extremal para a Conjectura 1.11.

O problema da densidade local em grafos visa estender o teorema de Turán, mas em contraste com este, onde o grafo extremal $\mathrm{T}_{n, r}$ é único, o fato que existem pelo menos duas famílias de possíveis grafos extremais para a Conjectura $1.2 \mathrm{com}$ propriedades estruturais tão diferentes, permite intuir que a Conjectura 1.2 poderia estar ainda longe de nosso alcance. Enfatizamos, por exemplo, que $\chi\left(\mathrm{C}_{5}^{[n]}\right)=3$ e $\chi\left(\mathrm{GPet}^{[n]}\right)=3$ mas, enquanto $\delta\left(\mathrm{C}_{5}^{[n]}\right)>n / 3$ contudo $\delta\left(\right.$ GPet $\left.^{[n]}\right)<n / 3$ e enquanto $\mathrm{C}_{5}^{[n]} \rightarrow \Gamma_{2}$ contudo ${ }^{\mathrm{GPet}}{ }^{[n]}$ não é homomorfo a algum grafo de Andrásfai $\Gamma_{i}$. Sudakov, quem tem estudado o problema, sugere que é possível que novas técnicas sejam necessárias para resolvê-la (cf. [Sud11, §5.1]).

Nossas provas usam argumentos padrões como a desigualdade de Cauchy-Schwarz, contagem dupla, álgebra linear, etc. Nosso resultado principal no Capítulo 2 usa uma aproximação análoga a aquela em Krivelevich [Kri95]. A menos de poucas excepções, várias das melhores cotas conhecidas para $\beta\left(\alpha, \mathrm{K}_{3}\right)$ nesta data, não revelam provas altamente técnicas. O Teorema 4.2 que melhora o resultado de Erdős, Faudree,Rousseau e Schelp [EFRS94, Theorem 2.4] é um exemplo. No entanto, nossos resultados conseguem melhoras apenas modestas, exceto nos casos onde estudamos propriedades estruturais para uma subclasse específica de grafos como no Capítulo 3.

No Capítulo 2, nós mostramos com o Teorema 1.3 que um grafo G de ordem $n$ com ogirth(G) $>5$ satisfaz $e_{1 / 2}(G) \leq n^{2} / 64$. Para infinitos valores de $n$, nós achamos grafos livres de triângulo de ordem $n$ que satisfazem $e_{1 / 2}(\mathrm{G})=n^{2} / 64$, cf. e.g. o grafo $\mathrm{GWag}^{[n]}$ e a relação (1.4) na página 4 ou o grafo ${ }_{\mathrm{GCl}} \mathrm{e}^{[n]}$ e a relação (4.1) na página 30. Porém, nós falhamos em achar grafos livres de triângulos e pentágonos que atinjam esta igualdade. De fato, a constante 1/64 no Teorema 1.3 poderia não ser justa devido a um comentário de Erdős no parágrafo final do Problema 1 em [Erd84]. Erdős perguntou se ogirth $(G)=2 k+1$ implica $e_{1 / 2}(G) \leq n^{2} /\left(2(2 k+1)^{2}\right)$ ? De ser verdade, isto é o melhor possível como mostra $\mathrm{C}_{2 k+1}^{[n]} \operatorname{com} 2(2 k+1) \mid n$.

Por outro lado, é razoável conjecturar que a constante 1/54 no Teorema 1.5 é o melhor possível ainda em grafos não regulares, i.e. nós conjecturamos que a declaração do Teorema 1.5 (provado no Capítulo 3) continua valendo ao remover a condição de regularidade de G.

Conjectura 5.1. Seja G um grafo de ordem n livre de triângulo com grau mínimo $\delta(\mathrm{G})>n / 3$. Se 
$\chi(\mathrm{G}) \neq 3$ então $\boldsymbol{e}_{1 / 2}(\mathrm{G})<n^{2} / 54$.

Conjectura 5.2. Para todo $\varepsilon>0$ existe um grafo $\mathrm{G}$ de ordem $n=n(\varepsilon)$ livre de triângulo com grau mínimo $\delta(\mathrm{G})>n / 3$ e número cromático $\chi(\mathrm{G})=4$ satisfazendo

$$
e_{1 / 2}(G) \geq\left(\frac{1}{54}-\varepsilon\right) n^{2} .
$$

Experimentações com bases de dados de grafos $\mathrm{K}_{3}$-livres sugerem que, fixado um núcleo H, blowups que minimizam o grau máximo fornecem densidade local maior. Um resultado nesta direção é desejável. É razoável supor que os grafos Vega atingem a maior densidade local justamente nos blow-ups desbalanceados que minimizam o grau máximo, e como observado em [BT10], estes blowup correspondem a funções de probabilidade sobre os vértices que maximizam o grau mínimo e tornam o grafo pesado regular com uma função de peso única.

No problema da densidade local em grafos, estivemos principalmente focados no caso particular $\beta\left(1 / 2, \mathrm{~K}_{3}\right)$ e relativamente poucas variantes deste caso foram consideradas. A sugestão de considerar grafos com cintura ímpar grande foi dada ao autor pelo Prof. Dr. Guilherme Oliveira Mota e o resultado principal no Capítulo 2 consegue responder uma primeira pergunta dele. Em grafos densos, o resultado de Kövári, Sós e Turán [KST54] e Bondy e Simonovits [BS74] impedem proibir grafos bipartidos ou ciclos pares. Grafos esparsos poderiam revelar dificuldades ainda maiores. Desde que $R(3, t)=\Theta\left(t^{2} / \log t\right)$ (cf. [Kim95]), grafos livres de triângulo de ordem $n$ tem número independente $\alpha(\mathrm{G})=\Omega(\sqrt{n \log n})$, isto implica que para alguma constante $c>0$ grafos nos quais subconjuntos de $\lfloor c \sqrt{n \log n}\rfloor$ vértices induzem pelo menos uma aresta contêm triângulos, assim, não podemos substituir $\lfloor\alpha n\rfloor$ por uma expressão sublinear arbitrária, no entanto, substituir $\lfloor\alpha n\rfloor$ por $\left\lfloor n^{1-\varepsilon}\right\rfloor$ poderia ser de interesse. Em vista dos resultados de de Caen e Füredi [dCF00], Füredi e Simonovits [FS05] e Keevash e Sudakov [KS05], para hipergrafos, proibir o plano de Fano é um razoável ponto de partida. 


\section{Apêndice A}

\section{Prova computacional do Teorema 1.8}

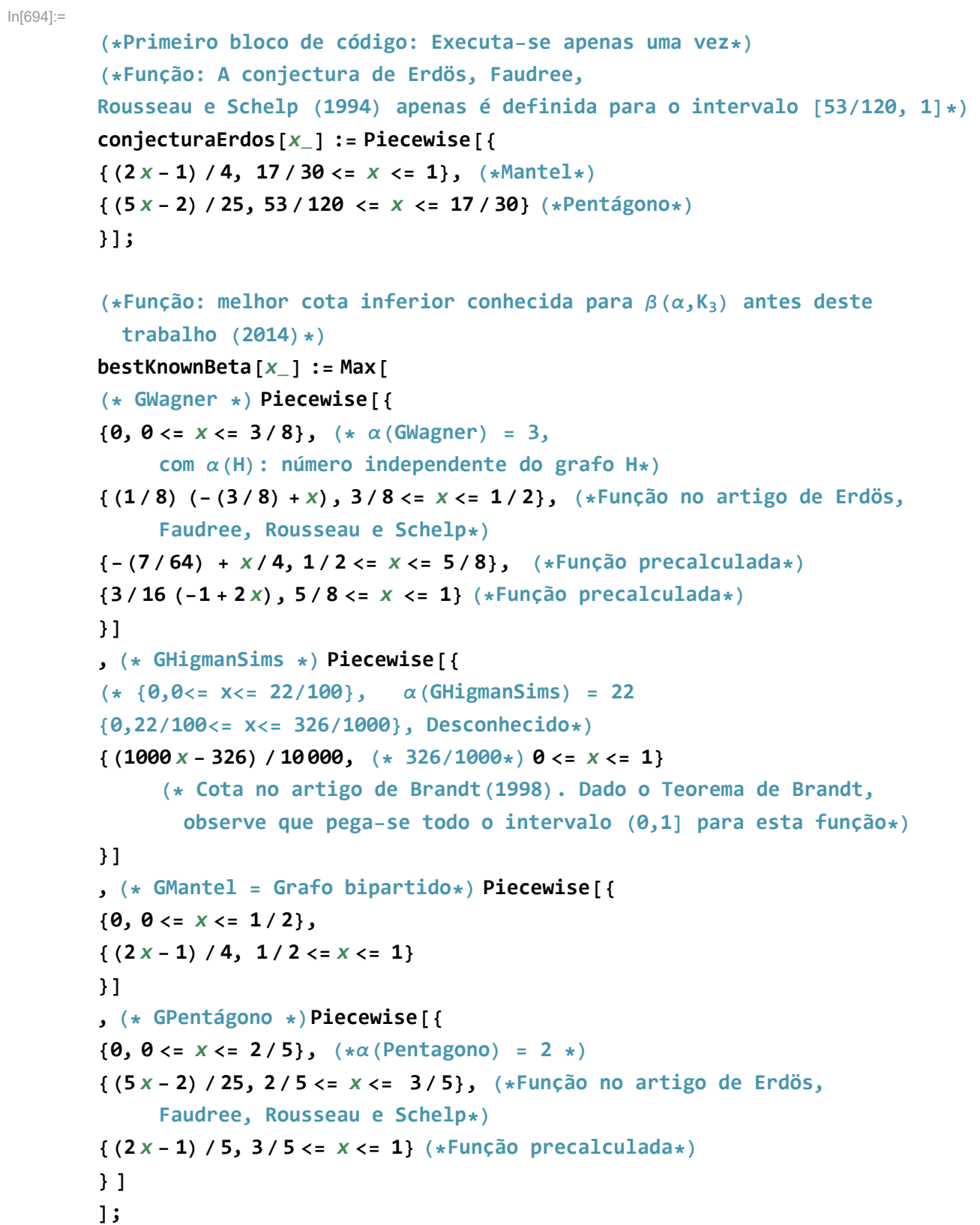


(*Segundo bloco de código: pode ser executado qualquer número de vezes.

Este bloco constrói a função contruyeBeta para o grafo de entrada

(neste caso, GClebsch) definida como segue*)

(*A função contruyeBeta $[\alpha]=\beta^{\prime}(G)=\operatorname{Min}\left[e_{\alpha}(U) / n^{2}: U\right.$ subset $\left.V(G),|U|=\alpha n\right] *$ )

(*Precisa-se limpar dados da execução anterior

(util depois da primeira execução do código)*)

ClearAll $[\beta$, construyeBeta, $\alpha]$;

(*Nome do grafo na base de dados GraphData[] da Wolfram*)

graphName = "ClebschGraph";

(*obtemos o grafo*)

G = GraphData [ graphName ] ;

(*Número indepente ou uma boa cota inferior*)

ind = GraphData [graphName, "IndependenceNumber"] ; ( * Se desconocido, use Max@VertexDegree[G]; como cota inferior para número independente*)

(*Ordem do grafo*)

$\mathbf{n}=\operatorname{VertexCount}[\mathrm{G}]$;

(*Analise do grafo

fracionário: precisa-se os intevalos da forma $[a / n,(a+1) / n]$ onde $a=$

Floor [ $\alpha \mathrm{n}]$ para todo $0<\alpha \leq 1$. Salvo o caso $a<$ ind $/ n$,

onde o mínimo número de arestas de subgrafos induzidos é zero.*)

intervalos $=\operatorname{Table}[\{($ ind +1$) / n,($ ind $+1+1) / n\},\{1,0, n-$ ind -1$\}]$;

(*Garante que apagamos valores, definição, atributos, etc.*)

Remove $[\beta$, construyeBeta, $\alpha]$;

(*Unprotect $[\beta$, construyeBeta, $\alpha] ; *$ )

( *SetAttributes [ construyeBeta, HoldFirst] ; *)

(*A função $\beta^{\prime}(\mathrm{G})$ evalua zero se $\alpha \mathrm{n} \leq$ número independente de $\mathrm{G} *$ )

$\beta\left[s_{-} / ; 0 \leq s \leq\right.$ Evaluate [ind / $\left.\left.\mathrm{n}\right]\right]:=0$;

(*Nós gostariamos ver os intervalos*)

Print $[\{\{\theta$, ind / $n\}, \theta\}]$;

(*A função $\beta^{\prime}(\mathrm{G})$ definida para $\alpha$ n >

número independente de G (veja execução de ParallelMap enseguida )*)

construyeBeta [interv_] :=

Module [ $\{$ inicioIntervalo, finIntervalo, u, s, minAristas, subGrafoEsparso,

maxGradoSubGrafo\},

inicioIntervalo = interv [[1] ];

finIntervalo = interv [ [2] ];

$\mathrm{u}=$ finIntervalo $\mathbf{n}$;

$s=\operatorname{Subset}[\operatorname{VertexList}[G],\{u\}] ;$

minAristas $=\operatorname{Min}[\operatorname{EdgeCount}[\#] \& / @(\operatorname{Subgraph}[G, \#] \& / @ s)]$;

maxGradoSubGrafo =

$\operatorname{Max}[$ VertexDegree [\#] \&/@ Select [Subgraph [G, \#] \&/@s,

EdgeCount $[\#]==$ minAristas \&] ] ;

subGrafoEsparso = SelectFirst $[\operatorname{Subgraph}[\mathrm{G}, \#] \& / @ \mathrm{~s}$,

EdgeCount $[\#]==\operatorname{minAristas} \& \& \operatorname{Max}[$ VertexDegree $[\#]$ ] == maxGradoSubGrafo \&];

(*HighlightGraph [G, subGrafoEsparso] *)

(* Print [ $\{$ inicioIntervalo, finIntervalo $\}$, (minAristas-maxGradoSubGrafo) $(1 / \mathrm{n})^{\wedge} 2+$

( $\alpha$-inicioIntervalo) (1/n) maxGradoSubGrafo\}//Simplify]; *)

$\beta\left[s_{-} /\right.$; inicioIntervalo $\leq s \leq$ finIntervalo] :=

Simplify [ (minAristas - maxGradoSubGrafo) $(\mathbf{1} / \mathbf{n})^{\wedge} \mathbf{2}+$

( $\alpha$ - inicioIntervalo) (1/n) maxGradoSubGrafo];

\section{Return [}

$\{$ inicioIntervalo, finIntervalo\},

(minAristas - maxGradoSubGrafo) $(1 / n) \wedge 2+$

( $\alpha$ - iniciolntervalo) (1/n) maxGradoSubGrafo\} // Simplify]; 
(*Análise dos intervalos en paralelo *)

ParallelMap [construyeBeta [\#] \&, intervalos];

(*Precisamos dar formato à saida para construir uma Piecewise*)

With $[\{$ int $=$ First $[\#], f=\operatorname{Last}[\#]\}$,

$\{f$, First [int $] \leq \alpha \leq$ Last [int] $\}$

] \&/@\%;

Block $[\{f\}$,

(*Nós gostariamos ver a função construida e dar um nome para ela: $f[\alpha] *$ )

Print [TableForm [\%] ];

$f\left[\alpha_{-}\right]:=$Piecewise $[\%]$;

(*Fazemos um Plot e comparamos com a Conjectura de Erdös et. al. como

também com a função de Brandt*)

\{Block [ \{plot, point\},

point $=$ Graphics $[\{\operatorname{Text}[53 / 120,\{53 / 120,0.04\}], \operatorname{Text}[1 / 2,\{1 / 2,0.04\}]\}]$;

plot $=\operatorname{Plot}[\{$ bestKnownBeta $[\alpha]$, conjecturaErdos $[\alpha], f[\alpha]\},\{\alpha, 1 / 3,3 / 5\}$,

PlotLegends $\rightarrow$ "Expressions",

Ticks $\rightarrow(\{(\{\#, N[\#, 2]\}$ \& / Range $[1 / 3,3 / 5,(2 / 75)]) \sim$ Union

$\{\{53 / 120, " ",\{0.4,0.03\}$, Directive [Dashed] $\}$,

$\{1 / 2, " ",\{0.4,0.03\}$, Directive $[$ Dashed $]\}\}$, Automatic $\}$

];

Show [plot, point

$\{" f[\alpha]>$ conjecturaErdos $[\alpha] "$

Reduce $[\{f[\alpha]>$ conjecturaErdos $[\alpha] \& \& 53 / 120<\alpha \leq 1\},\{\alpha\}]\}$,

$\{" f[\alpha]>$ bestKnownBeta $[\alpha]$ (Brandt) ",

Reduce $[\{[\alpha]>$ bestKnownBeta $[\alpha] \& \& 5 / 16<\alpha \leq 1\},\{\alpha\}]\}$

$\left\{\left\{0, \frac{5}{16}\right\}, 0\right\}$

$\frac{1}{8}\left(-\frac{5}{16}+\alpha\right.$

$\frac{1}{64}(-1+4 \alpha)$

$\frac{5}{16} \leq \alpha \leq \frac{3}{8}$

$\frac{1}{64}(-1+4 \alpha)$

$-\frac{7}{64}+\frac{\alpha}{4}$

$-\frac{7}{64}+\frac{\alpha}{4}$

$\frac{3}{128}(-3+8 \alpha)$

$\frac{5}{32}(-1+2 \alpha)$

$\frac{5}{32}(-1+2 \alpha)$

$\frac{5}{32}(-1+2 \alpha)$

$\frac{5}{32}(-1+2 \alpha)$

$\frac{5}{32}(-1+2 \alpha)$

$\frac{3}{8} \leq \alpha \leq \frac{7}{16}$

$\frac{7}{16} \leq \alpha \leq \frac{1}{2}$

$\frac{1}{2} \leq \alpha \leq \frac{9}{16}$

$\frac{9}{16} \leq \alpha \leq \frac{5}{8}$

$\frac{5}{8} \leq \alpha \leq \frac{11}{16}$

$\frac{11}{16} \leq \alpha \leq \frac{3}{4}$

$\frac{3}{4} \leq \alpha \leq \frac{13}{16}$

$\frac{13}{16} \leq \alpha \leq \frac{7}{8}$

$\frac{7}{8} \leq \alpha \leq \frac{15}{16}$

$\frac{15}{16} \leq \alpha \leq 1$

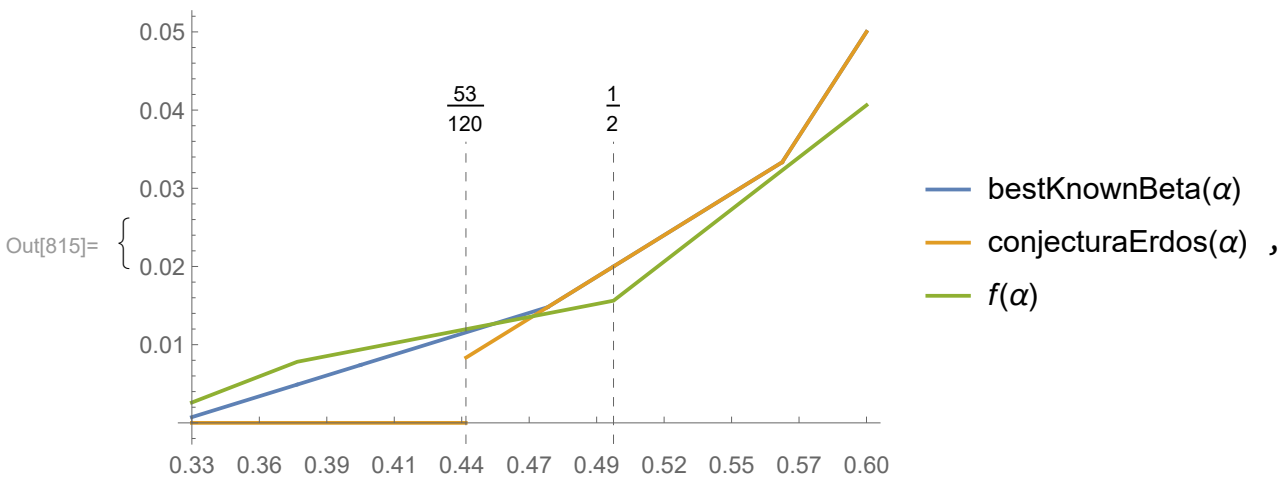

$\left\{f[\alpha]>\right.$ conjecturaErdos $\left.[\alpha], \frac{53}{120}<\alpha<\frac{103}{220}\right\},\left\{f[\alpha]>\right.$ bestKnownBeta $[\alpha] \quad($ Brandt $\left.\left.), \frac{5}{16}<\alpha<\frac{679}{1500}\right\}\right\}$ 
APÊNDICE A 


\section{Referências Bibliográficas}

$\left[\mathrm{ABG}^{+} 13\right]$ Peter Allen, Julia Böttcher, Simon Griffiths, Yoshiharu Kohayakawa e Robert Morris. The chromatic thresholds of graphs. Advances in Mathematics, 235:261-295, 2013. 21

[ABKS03] Noga Alon, Béla Bollobás, Michael Krivelevich e Benny Sudakov. Maximum cuts and judicious partitions in graphs without short cycles. Journal of Combinatorial Theory, Series B, 88(2):329-346, jul 2003. 12

[ACH93] Michael O. Albertson, Lily Chan e Ruth Haas. Independence and graph homomorphisms. Journal of Graph Theory, 17(5):581-588, nov 1993. 15

[AES74] Béla Andrásfai, Paul Erdős e Vera Sós. On the connection between chromatic number, maximal clique and minimal degree of a graph. Discrete Mathematics, 8(3):205-218, may 1974. 14

[AKS80] Miklós Ajtai, János Komlós e Endre Szemerédi. A note on Ramsey numbers. Journal of Combinatorial Theory, Series A, 29(3):354-360, nov 1980. 29

[Alo96] Noga Alon. Bipartite subgraphs. Combinatorica, 16(3):301-311, sep 1996. 12

[And62] Béla Andrásfai. Über ein extremalproblem der graphentheorie. Acta Mathematica Academiae Scientiarum Hungaricae, 13:443-455, 1962. 13

[AS92] Noga Alon e Joel H. Spencer. The probabilistic method. John Wiley \& Sons Inc., New York, 1992. With an appendix by Paul Erdős, A Wiley-Interscience Publication. 8

[BBH98] Stephan Brandt, Gunnar Brinkmann e Thomas Harmuth. All Ramsey numbers $r\left(K_{3}, G\right)$ for connected graphs of order 9. The Electronic Journal of Combinatorics, 5(1):research paper $\mathrm{r} 7,20,1998.5$

[BBH00] Stephan Brandt, Gunnar Brinkmann e Thomas Harmuth. The generation of maximal triangle-free graphs. Graphs and Combinatorics, 16(2):149-157, 2000. 5

[BF92] László Babai e Péter Frankl. Linear algebra methods in combinatorics, with applications to geometry and computer science (Preliminary version 22 ). University of Chicago, 09 1992. 8

[BK13] Tom Bohman e Peter Keevash. Dynamic concentration of the triangle-free process. Em The seventh European conference on combinatorics, graph theory and applications. Extended abstracts of EuroComb 2013, Pisa, Italy, September 9-13, 2013, páginas 489495. Pisa: Edizioni della Normale, 2013. 30

[BM11] John Adrian Bondy e Uppaluri Siva Ramachandra Murty. Graph Theory, volume 244 of Graduate Texts in Mathematics. Springer London Ltd, 2011. 6

[BMRS17] Wiebke Bedenknecht, Guilherme Oliveira Mota, Christian Reiher e Mathias Schacht. On the local density problem for graphs of given odd-girth. Em LAGOS 201\%. Selected papers of the 9th Latin-American algorithms, graphs, and optimization symposium, 
Marseille, France, September 11-15, 2017, páginas 39-44. Amsterdam: Elsevier, 2017. $3,11,14,15,22$

[Bol98] Béla Bollobás. Modern graph theory, volume 184. New York, NY: Springer, 1998. 6

[Bol04] Béla Bollobás. Extremal graph theory. Mineola, NY: Dover Publications, reprint of the 1978 original edição, 2004. Reprint of the 1978 original. 8

[BP98] Stephan Brandt e Tomaž Pisanski. Another infinite sequence of dense triangle-free graphs. The Electronic Journal of Combinatorics, 5(1):research paper r43, 5, 1998. 23

[Bra98] Stephan Brandt. The local density of triangle-free graphs. Discrete Mathematics, 183(13):17-25, mar 1998. 4, 5, 11, 30

[Bra99] Stephan Brandt. On the structure of dense triangle-free graphs. Combinatorics Probability and Computing, 8(3):237-245, 1999. 3, 21, 22

[Bra02] Stephan Brandt. A 4-colour problem for dense triangle-free graphs. Discrete Mathematics, 251(1-3):33-46, may 2002. 13

[BRB09] Stephan Brandt e Elizabeth Ribe-Baumann. Graphs of odd girth 7 with large degree. Electronic Notes in Discrete Mathematics, 34:89-93, aug 2009. 15

[Bri98] Gunnar Brinkmann. All Ramsey numbers $r\left(K_{3}, G\right)$ for connected graphs of order 7 and 8. Combinatorics, Probability and Computing, 7(2):129-140, 1998. 5

[BS74] John Adrian Bondy e Miklós Simonovits. Cycles of even length in graphs. Journal of Combinatorial Theory, Series B, 16(2):97-105, apr 1974. 34

[BS95] V.I. Baranov e B. S. Stechkin. Extremal combinatorial problems and their applications. Dordrecht: Kluwer Academic Publishers, 1995. (Transl. from the Russian). 1

[BS99] Béla Bollobás e Alexander Scott. Exact bounds for judicious partitions of graphs. Combinatorica, 19(4):473-486, 1999. 12

[BS02] Béla Bollobás e Alexander Scott. Better bounds for Max Cut. Em Contemporary combinatorics. Collection of survey papers in combinatorics based on lectures given in the workshop on probabilistic combinatorics at the Paul Erdös Summer Research Center in Mathematics, Budapest, Hungary, páginas 185-246. Berlin: Springer; Budapest: János Bolyai Mathematical Society, 2002. 11

[BT10] Stephan Brandt e Stéphan Thomassé. Dense triangle-free graphs are four-colorable: A solution to the Erdős-Simonovits problem. preprint, 2010. 3, 21, 22, 23, 24, 34

[CDS95] Dragoš M. Cvetković, Michael Doob e Horst Sachs. Spectra of graphs: theory and applications. Leipzig: J. A. Barth Verlag, 3rd rev. a. enl. ed. edição, 1995. 8

[CG90] Fan R. K. Chung e Ronald L. Graham. On graphs not containing prescribed induced subgraphs. Em Generating expanders from two permutations, páginas 111-120. 1990. 9

[CG99] Fan R. K. Chung e Ronald L. Graham. Erdös on graphs. His legacy of unsolved problems. Wellesley, MA: A K Peters, 1999. 8

[Chv74] Vašek Chvátal. The minimality of the Mycielski graph. Em Lecture Notes in Mathematics, páginas 243-246. Springer Nature, 1974. 3

[CJK97] Chuan-Chong Chen, Guoping P. Jin e Khee Meng Koh. Triangle-free graphs with large degree. Combinatorics, Probability and Computing, 6(4):381-396, dec 1997. 3, 21, 22, 23 
[Cle68] Alfred Clebsch. Ueber die Flächen vierter Ordnung, welche eine Doppelcurve zweiten Grades besitzen. J. Reine Angew. Math., 69:142-147, 1868. 29

[dCF00] Dominique de Caen e Zoltán Füredi. The maximum size of 3-uniform hypergraphs not containing a Fano plane. Journal of Combinatorial Theory, Series B, 78(2):274-276, mar 2000. 34

[Des48] Blanche Descartes. A three color problem. Eureka (April 1947); Solution (March 1948)., 1948. 9,21

[Die10] Reinhard Diestel. Graph Theory, volume 173 of Graduate Texts in Mathematics. Springer Berlin Heidelberg, Heidelberg, fourth edição, 2010. 6

[Edw73] C. Scott Edwards. Some extremal properties of bipartite subgraphs. Journal canadien de mathématiques, 25(0):475-485, jan 1973. 11

[Edw75] C. Scott Edwards. An improved lower bound for the number of edges in a largest bipartite subgraph. Recent Adv. Graph Theory, Proc. Symp. Prague 1974, 167-181 (1975)., 1975. 11

[EFPS88] Paul Erdős, Ralph Jill Faudree, János Pach e Joel H. Spencer. How to make a graph bipartite. Journal of Combinatorial Theory, Series B, 45(1):86-98, aug 1988. 11

[EFRS94] Paul Erdős, Ralph Jill Faudree, Cecil C. Rousseau e Richard H. Schelp. A local density condition for triangles. Discrete Mathematics, 127(1-3):153-161, mar 1994. 4, 5, 11, 13, $29,30,33$

[EGK97] Paul Erdős, András Gyárfás e Yoshiharu Kohayakawa. The size of the largest bipartite subgraphs. Discrete Math., 177(1-3):267-271, 1997. 11

[EGS92] Paul Erdős, Ervin Győri e Miklós Simonovits. How many edges should be deleted to make a triangle-free graph bipartite? Em Sets, graphs and numbers. A birthday salute to Vera T. Sós and András Hajnal, páginas 239-263. Amsterdam: North-Holland Publishing Company, 1992. 11

[EKR76] Paul Erdős, Daniel J. Kleitman e Bruce L. Rothschild. Asymptotic enumeration of $K_{n}$-free graphs. Em Colloquio Internazionale sulle Teorie Combinatorie (Rome, 1973), Tomo II, páginas 19-27. Accad. Naz. Lincei, 1976. Atti dei Convegni Lincei, No. 17. 9

[Erd59] Paul Erdős. Graph theory and probability. Journal canadien de mathématiques, 11(0):34-38, jan 1959. Available at:https://users.renyi.hu/ p_erdos/ 1959-06.pdf. 10

[Erd61] Paul Erdős. Graph theory and probability II. Canadian Journal of Mathematics, 13:346-352, 1961. 29

[Erd62] Paul Erdős. Applications of probability to combinatorial problems. Colloq. Comb. Methods Probab. Theor., Aarhus 1962, 90-92 (1962), 1962. Available at: https:// users.renyi.hu/ p_erdos/1962-02.pdf. 9, 21

[Erd64] Paul Erdős. Some applications of probability to graph theory and combinatorial problems. Theory Graphs Appl., Proc. Symp. Smolenice 1963, 133-136 (1964), 1964. URL: https://users.renyi.hu/ p_erdos/1964-26.pdf.9

[Erd71] Paul Erdős. Some unsolved problems in graph theory and combinatorial analysis. Em Combinatorial Mathematics and its Applications (Proc. Conf., Oxford, 1969), páginas 97-109. Academic Press, London, 1971. Available at:https://users.renyi.hu/ p_erdos/1971-25.pdf. 4, 10 
[Erd76] Paul Erdös. Problems and results in graph theory and combinatorial analysis. Em 5th British Combinatorial Conference Aberdeen (Scotland, 1975), number XV in Congresssus Numeratium, páginas 169-192. Utilitas Math. Winnipeg, Man., 1976. 1, 2, 8, 10

[Erd84] Paul Erdős. On some problems in graph theory, combinatorial analysis and combinatorial number theory. Em B. Bollobás, editor, Graph theory and combinatorics (Cambridge, 1983), páginas 1-17, London, New York, 1984. Academic Press. Available at:https://users.renyi.hu/ p_erdos/1984-11.pdf. 10,13, 33

[Erd97] Paul Erdös. Some old and new problems in various branches of combinatorics. Discrete Mathematics, 165-166(15):227-231, mar 1997. 2, 4, 13

[ES35] Paul Erdős e George Szekeres. A combinatorial problem in geometry. Compositio Mathematica, 2:463-470, 1935. 29

[ES73] Paul Erdős e Miklós Simonovits. On a valence problem in extremal graph theory. Discrete Mathematics, 5(4):323-334, aug 1973. 3, 21

[FG05] S. Fajtlowicz e DIMACS (Group). Graphs and Discovery, volume 69 of DIMACS series in discrete mathematics and theoretical computer science. American Mathematical Society, 2005. 23

[FS05] Zoltán Füredi e Miklós Simonovits. Triple systems not containing a Fano configuration. Combinatorics, Probability and Computing, 14(4):467-484, jul 2005. 34

[GL11] Wayne Goddard e Jeremy Lyle. Dense graphs with small clique number. Journal of Graph Theory, 66(4):319-331, 2011. 21

[GR01] Chris Godsil e Gordon F. Royle. Algebraic Graph Theory, volume 207 of Graduate Texts in Mathematics. Springer New York, 2001, 2001. 8, 13, 14

[GRS13] Ronald L. Graham, Bruce L. Rothschild e Joel H. Spencer. Ramsey theory. With a new foreword. Reprint of the 1990 original 2nd edition. Hoboken, NJ: John Wiley \& Sons, reprint of the 1990 original 2nd edition edição, 2013. 8, 29

[Grz12] Andrzej Grzesik. On the maximum number of five-cycles in a triangle-free graph. Journal of Combinatorial Theory, Series B, 102(5):1061-1066, sep 2012. 10

[GY68] Jack E. Graver e James Yackel. Some graph theoretic results associated with Ramsey's theorem. Journal of Combinatorial Theory, 4:125-175, mar 1968. 29

[Häg82] Roland Häggkvist. ODD cycles of specified length in non-bipartite graphs. Em Graph Theory, Proceedings of the Conference on Graph Theory, volume 62 of North-Holland Mathematics Studies, páginas 89-99. North-Holland, 1982. 14, 21

$\left[\mathrm{HHK}^{+} 13\right]$ Hamed Hatami, Jan Hladký, Daniel Král', Seguei Norine e Alexander A. Razborov. On the number of pentagons in triangle-free graphs. Journal of Combinatorial Theory, Series A, 120(3):722-732, apr 2013. 10

[HJ98] Roland Häggkvist e Guoping P. Jin. Graphs with odd girth at least seven and high minimum degree. Graphs and Combinatorics, 14(4):351-362, nov 1998. 15

[HL98] Thomas Hofmeister e Hanno Lefmann. On $k$-partite subgraphs. Ars Comb., 50:303-308, 1998. 11

[HN92] Pavol Hell e Jaroslav Nešetřil. The core of a graph. Discrete Mathematics, 109(13):117-126, 1992. 22 
[HS68] Donald G. Higman e Charles C. Sims. A simple group of order 44,352,000. Mathematische Zeitschrift, 105(2):110-113, 1968. 5

[HSW11] John Edward Hopcroft, Sucheta Soundarajan e Liaoruo Wang. The future of computer science. International Journal of Software and Informatics, 5(4):549-565, 2011. 1

[Jin93] Guoping P. Jin. Triangle-free graphs with high minimal degrees. Combinatorics, Probability and Computing, 2(04):479-490, dec 1993. 3, 14, 21

[Kar72] Richard M. Karp. Reducibility among combinatorial problems. Em Complexity of Computer Computations, páginas 85-103. Springer US, 1972. 11

[Kim95] Jeong Han Kim. The Ramsey number $R(3, t)$ has order of magnitude $t^{2} / \log t$. Random Structures \& Algorithms, 7(3):173-207, 1995. 29, 34

[KK54] John B. Kelly e L. M. Kelly. Paths and circuits in critical graphs. American Journal of Mathematics, 76:786-792, 1954. 9

[Kri95] Michael Krivelevich. On the edge distribution in triangle-free graphs. Journal of Combinatorial Theory, Series B, 63(2):245-260, mar 1995. 5, 11, 13, 16, 22, 30, 33

[KS03] Peter Keevash e Benny Sudakov. Local density in graphs with forbidden subgraphs. Combinatorics, Probability and Computing, 12(02):139-153, mar 2003. 9, 11, 30

[KS05] Peter Keevash e Benny Sudakov. The Turán number of the Fano plane. Combinatorica, 25(5):561-574, sep 2005. 34

[KS06] Peter Keevash e Benny Sudakov. Sparse halves in triangle-free graphs. Journal of Combinatorial Theory, Series B, 96(4):614-620, jul 2006. 3, 11, 17, 18

[KST54] Tamás Kövári, Vera Sós e Paul Turán. On a problem of K. Zarankiewicz. Colloquium Mathematicum, 3:50-57, 1954. 34

[KW17] Mikhail H. Klin e Andrew J. Woldar. The strongly regular graph with parameters $(100,22,0,6)$ : Hidden history and beyond. Acta Universitatis Matthiae Belii. Series Mathematics, 25:5-62, 2017. 5

[Loc82] S.C. Locke. Maximum k-colorable subgraphs. Journal of Graph Theory, 6:123-132, 1982. 11

[LP17] Bernard Lidický e Florian Pfender. Pentagons in triangle-free graphs. ArXiv e-prints, página arXiv:1712.08869, Dezembro 2017. 10, 11

[LS18] Shoham Letzter e Richard Snyder. The homomorphism threshold of $\left\{C_{3}, C_{5}\right\}$-free graphs. Journal of Graph Theory, jun 2018. 15

[LT82] Jenö Lehel e Zsolt Tuza. Triangle-free partial graphs and edge covering theorems. Discrete Math., 39:59-65, 1982. 11

[Man07] Willem Mantel. Vraagstuk XXVIII. Wiskundige Opgaven, 10:60-61, 1907. 1, 4, 8

[Mes56] Dale Marsh Mesner. An investigation of certain combinatorial properties of partially balanced incomplete block experimental designs and association schemes, with a detailed study of designs of Latin square and related types. Tese de Doutorado, Michigan State University, 1956. 5

[Mes64] Dale Marsh Mesner. Negative Latin square designs. Mimeo Series, Institute of Statistics, 40, 1964. 5 
[Mic13] T. S. Michael. Cycles of length 5 in triangle-free graphs: a sporadic counterexample to a characterization of equality. Bulletin of the Institute of Combinatorics and its Applications, 67:6-8, 2013. 10

[MS14] Silvia Messuti e Mathias Schacht. On the structure of graphs with given odd girth and large minimum degree. Journal of Graph Theory, 80(1):69-81, nov 2014. 15

[Myc55] Jan Mycielski. Sur le coloriage des graphes. Em Colloq. Math, volume 3, página 9, 1955. 9,21

[Nik10] Vladimir Nikiforov. Chromatic number and mimimum degree of $K_{r}$-free graphs. ArXiv e-prints, Janeiro 2010. 21

[NY13] Seguei Norine e Liana E. Yepremyan. Sparse halves in dense triangle-free graphs. Electronic Notes in Discrete Mathematics, 43:271-278, sep 2013. 11, 14

[Pis95] Tomaž Pisanski. Vega Version 0.2; Quick Reference Manual and Vega graph gallery, Ljubljana. 1995. vega.ijp.si. 23

[Por92] T.D. Porter. On a bottleneck bipartition conjecture of Erdős. Combinatorica, 12(3):317321, 1992. 12

[Rad94] Stanisław P. Radziszowski. Small Ramsey numbers. Electron. J. Combin, 1(7), 1994. 30

[Ram29] Frank Plumpton Ramsey. On a problem of formal logic. Proceedings of the London Mathematical Society. Second Series, 30:264-286, 1929. 8, 29

[She83] James B. Shearer. A note on the independence number of triangle-free graphs. Discrete Mathematics, 46(1):83-87, 1983. 30

[She91] James B. Shearer. A note on the independence number of triangle-free graphs, II. Journal of Combinatorial Theory, Series B, 53(2):300-307, nov 1991. 30

[She92] James B. Shearer. A note on bipartite subgraphs of triangle-free graphs. Random Structures \& Algorithms, 3(2):223-226, 1992. 30

[She95] James B. Shearer. The independence number of dense graphs with large odd girth. Electron. J. Combin, 2(2), 1995. 30

[Shi12] Rob Shields. Cultural topology: the seven bridges of Königsburg, 1736. Theory, Culture and Society, 29(4-5):43-57, jul 2012. 1

[SM45] Edward Szpilrajn-Marczewski. Sur deux propriétés des classes d'ensembles. Fundamenta Mathematicae, 33:303-307, 1945. 7

[Spe94] Joel H. Spencer. Ten lectures on the probabilistic method, volume 64. Philadelphia, PA: SIAM, 2nd ed. edição, 1994. 8

[SS94] Farhad Shahrokhi e László A. Székely. The complexity of the bottleneck graph bipartition problem. J. Combin. Math. Combin. Comp, 15(94):221-226, 1994. 12, 33

[SS01] Miklós Simonovits e Vera Sós. Ramsey-Turán theory. Discrete Mathematics, 229(13):293-340, 2001. 8

[Sud11] Benny Sudakov. Recent developments in extremal combinatorics: Ramsey and Turán type problems. Em Proceedings of the International Congress of Mathematicians 2010 (ICM 2010), páginas 2579-2606. World Scientific Pub Co Pte Lt, jun 2011. 2, 4, 8, 33 
[Tao12] Terence Tao. Topics in random matrix theory, volume 132. Providence, RI: American Mathematical Society (AMS), 2012. 7

[Tho02] Carsten Thomassen. On the chromatic number of triangle-free graphs of large minimum degree. Combinatorica, 22(4):591-596, oct 2002. 21

[Tur41] Paul Turán. Eine Extremalaufgabe aus der Graphentheorie. Mat. Fiz. Lapok, 48:436452, 1941. (Hungarian. German summary). 7, 8

[Tur54] Paul Turán. On the theory of graphs. Colloquium Mathematicum, 3:19-30, 1954. 9

[Wes05] Douglas B. West. Introduction to graph theory. New Delhi: Prentice-Hall of India, 2nd ed. edição, 2005. 6

[XLL18] Xiaodong Xu, Meilian Liang e Haipeng Luo. Ramsey theory. Unsolved problems and results. Berlin: De Gruyter, 2018. 8, 29

[Yan78] Mihalis Yannakakis. Node-and edge-deletion NP-complete problems. Em Proceedings of the Tenth Annual ACM Symposium on Theory of Computing, STOC '78, páginas 253-264, New York, NY, USA, 1978. ACM. 11, 33

[Zyk49] Alexander Aleksandrovich Zykov. On some properties of linear complexes. Matematicheskii sbornik, 66(2):163-188, 1949. 9, 21 